\title{
Rh(III)-Catalyzed Coupling of Acrylic Acids and Ynenones via Olefinic C-H Activation and Michael Addition
}

Yuqin Jiang, ${ }^{\dagger}$ Pengfei Li,${ }^{\dagger}$ Juanjuan Wang,${ }^{\dagger}$ Jie Zhao, ${ }^{\dagger}$ Yang Li,${ }^{\dagger}$ Yawen Zhang, ${ }^{\dagger}$ Junbiao Chang, ${ }^{* \dagger}$ Bingxian Liu, ${ }^{* \dagger}$ Xingwei Li ${ }^{*},+\dagger$

'Henan Key Laboratory of Organic Functional Molecule and Drug Innovation, Collaborative Innovation Center of Henan Province for Green Manufacturing of Fine Chemicals, School of Chemistry and Chemical Engineering, Henan Normal University, Xinxiang 453007, China ${ }^{*}$ School of Chemistry and Chemical Engineering, Shaanxi Normal University (SNNU), Xi'an 710062, China

X. L.: lixw@snnu.edu.cn; B. L.: liubx1120@163.com; J. C.: changjunbiao@zzu.edu.cn

\section{Table of Contents}

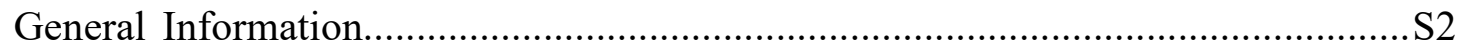

General Procedure for Preparation of Substrates 1-2 _............................................S2

General Procedures for The C-H alkenylation/Michael Addition Cascade .................S3

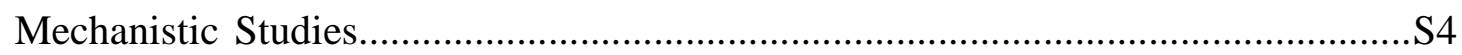

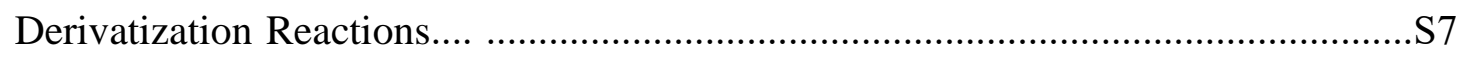

Antitumor Bio-activities of Selected Compounds..................................................... 9

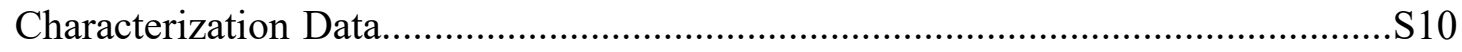

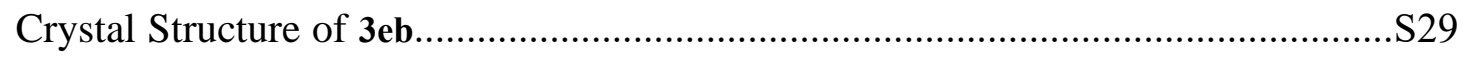

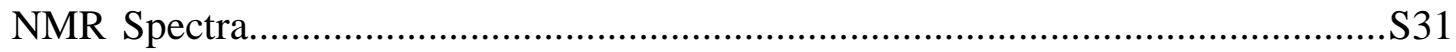

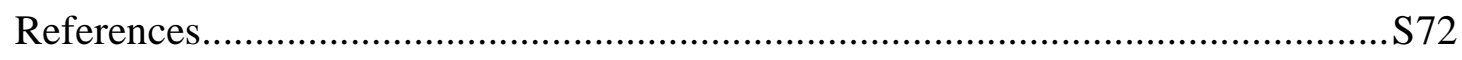




\section{General Information}

All the reactions were carried out under air atmosphere. The ${ }^{1} \mathrm{H}$ NMR spectra were recorded on a $400 \mathrm{MHz}$ or $600 \mathrm{MHz}$ NMR spectrometer. The ${ }^{13} \mathrm{C}$ NMR spectra were recorded at $100 \mathrm{MHz}$ or $150 \mathrm{MHz}$. The ${ }^{19} \mathrm{~F}$ NMR spectra were recorded at 376 or 565 MHz. The residual solvent signals were used as references and the chemical shifts were converted to the TMS scale. The coupling constants were given in Hz. HRMS data were obtained using a TOF mode. The conversion of starting materials was monitored by thin layer chromatography (TLC) using silica gel plates (silica gel 60 F254 $0.25 \mathrm{~mm}$ ), and components were visualized under UV light (254 and $365 \mathrm{~nm}$ ). Column chromatography was performed on silica gel 200-300 mesh. Unless otherwise noted, all other compounds have been reported in the literature or are commercially available. Commercial reagents were used without further purification.

\section{General Procedure for Preparation of Substrates 1-2}

Synthesis of substrate $\mathbf{1}^{1-2}$

Procedure A:

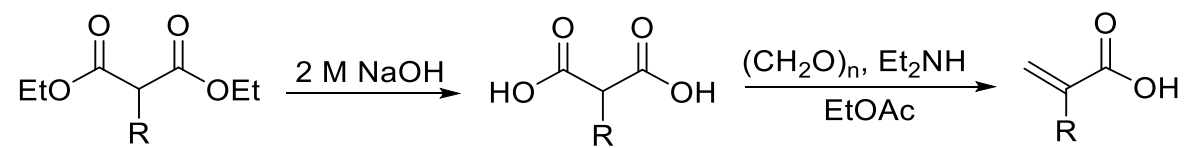

To a substituted malonate was added aq $\mathrm{NaOH}$ ( $2 \mathrm{M}, 2.67$ equiv), and the resulting mixture was stirred vigorously and refluxed for $2 \mathrm{~h}$ by oil bath. The resulting solution was cooled to rt and extracted with hexane and the aqueous layer was then acidified to $\mathrm{pH} 1$ with aq $\mathrm{HCl}$. The resulting solution was then extracted with EtOAc, and the combined organics was washed with brine, and dried with $\mathrm{Na}_{2} \mathrm{SO}_{4}$. The volatiles were removed in vacuo to afford the corresponding diacid which was used for the next step. The crude diacid was dissolved in EtOAc $(0.75 \mathrm{M})$ and the resulting solution was cooled to $0{ }^{\circ} \mathrm{C}$, followed by the dropwise addition of diethylamine (1.01 eq) and subsequent addition of paraformaldehyde (1.5 eq). The resulting suspension was refluxed for 2 hours by oil bath and then the reaction mixture was cooled to $0{ }^{\circ} \mathrm{C}$, diluted with $\mathrm{H}_{2} \mathrm{O}(0.6 \mathrm{~mL} / \mathrm{mmol}$ diacid $)$ and acidified to $\mathrm{pH} 1$ with concentrated $\mathrm{HCl}$. The aqueous layer was then extracted with EtOAc and the combined organics was washed with brine, and dried with $\mathrm{Na}_{2} \mathrm{SO}_{4}$. Then crude product was purified by column chromatography to provide the corresponding acid.

Procedure B: 


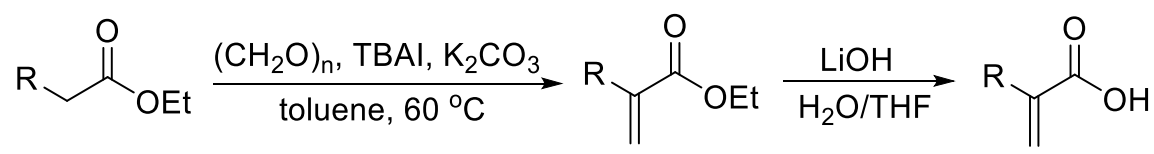

Paraformaldehyde (3.0 eq), $\mathrm{K}_{2} \mathrm{CO}_{3}$ (3.0 eq), and $n$-Bu4NI ( 0.05 eq) were added to a solution of ester (1.0 equiv) in toluene $(1 \mathrm{M})$ at $\mathrm{rt}$. The reaction mixture was stirred at $60{ }^{\circ} \mathrm{C}$ by oil bath for $8 \mathrm{~h}$. Then mixture was concentrated in vacuo and purified by column chromatography to provide the corresponding acrylate.

The acrylate ester was dissolved in $\mathrm{THF} / \mathrm{H}_{2} \mathrm{O}(0.25 \mathrm{M}, 1: 1)$ and $\mathrm{LiOH}$ (5 equiv) was subsequently added. The resulting suspension was refluxed for 8 hours at $80{ }^{\circ} \mathrm{C}$ by oil bath, and then the reaction mixture was cooled to $0{ }^{\circ} \mathrm{C}$, diluted with $\mathrm{H}_{2} \mathrm{O}$ and acidified to $\mathrm{pH} 1$ with concentrated $\mathrm{HCl}$. The aqueous layer was then extracted with EtOAc and the combined organics washed with brine, and dried with $\mathrm{Na}_{2} \mathrm{SO}_{4}$. The crude acid was purified by column chromatography.

Synthesis of substrate $2^{3}$

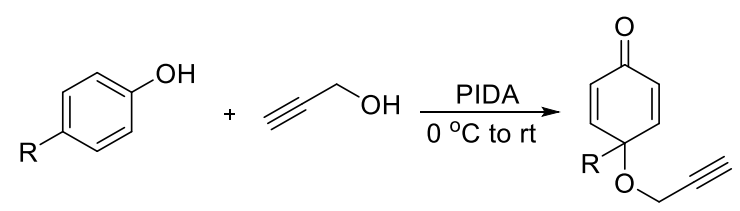

4-Substituted phenol (50 mmol) was dissolved in propargyl alcohol (100 $\mathrm{mL})$ and was stirred for $10 \mathrm{~min}$ at $0{ }^{\circ} \mathrm{C}$. Phenyliodine diacetate (PIDA, $50.0 \mathrm{mmol}$ ) was then added. The resulting mixture was warmed to room temperature and stirred $30 \mathrm{~min}$. Then it was quenched with saturated aqueous $\mathrm{NaHCO}_{3}(50 \mathrm{~mL})$ and extracted with EtOAc $(50 \mathrm{~mL}$ $\times 3)$. The combined organic phases were washed with brine $(100 \mathrm{~mL})$, dried over anhydrous $\mathrm{Na}_{2} \mathrm{SO}_{4}$ and concentrated under reduced pressure. The residue was purified by flash column chromatography using hexane/ethyl acetate eluent to afford the corresponding product 2.

\section{General Procedures for the $\mathrm{C}-\mathrm{H}$ alkenylation/Michael addition cascade}

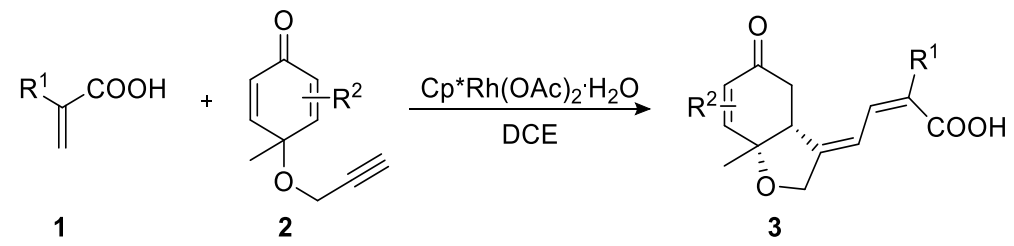

A mixture of acid $1(0.6 \mathrm{mmol}), \mathrm{Cp} * \mathrm{Rh}(\mathrm{OAc})_{2} \cdot \mathrm{H}_{2} \mathrm{O}(0.016 \mathrm{mmol}, 8.0 \mathrm{~mol} \%)$, and DCE $(6 \mathrm{~mL})$ were charged into a reaction tube. Then alkyne $2(0.2 \mathrm{mmol})$ was added in three portions $(0.067 \mathrm{mmol} / 3 \mathrm{~h})$. The reaction mixture was stirred under air at $60{ }^{\circ} \mathrm{C}$ by oil bath for totally $9 \mathrm{~h}$. After the solvent was removed under reduced pressure, the residue was purified by silica gel chromatography using PE/EA (2:1) to afford 3 . 


\section{Mechanism Studies}

H/D exchange experiments:<smiles>O=C(O)/C=C\Br</smiles>

$1 a$<smiles>C#CCOC1(C)C=CC(=O)C(C)=C1</smiles>

2b<smiles>O=[N+]([O-])[O-]</smiles>

1a-d

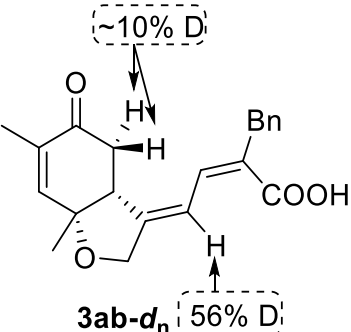

$3 a b-d_{n}: 56 \%$

A mixture of $\mathbf{1 a}\left(0.6 \mathrm{mmol}, 3\right.$ equiv), $\mathbf{2 b}(0.2 \mathrm{mmol}), \mathrm{Cp} * \mathrm{Rh}(\mathrm{OAc})_{2} \mathrm{H}_{2} \mathrm{O}(0.016 \mathrm{mmol}$, $8.0 \mathrm{~mol} \%)$, and $\mathrm{D}_{2} \mathrm{O}(10 \mathrm{eq})$ were added into a reaction tube. DCE $(6.0 \mathrm{~mL})$ was added and the mixture was stirred at $60^{\circ} \mathrm{C}$ by oil bath for $3 \mathrm{~h}$. Then the solvent was evaporated under reduced pressure and the residue was adsorbed onto small amounts of silica. The product 3ab- $\boldsymbol{d}_{\mathbf{n}}$ and the recovered 1a- $\boldsymbol{d}$ were obtained by flash column chromatography on silica gel (eluent: $\mathrm{PE} / \mathrm{EA}=6: 1$ to $2: 1$ ).

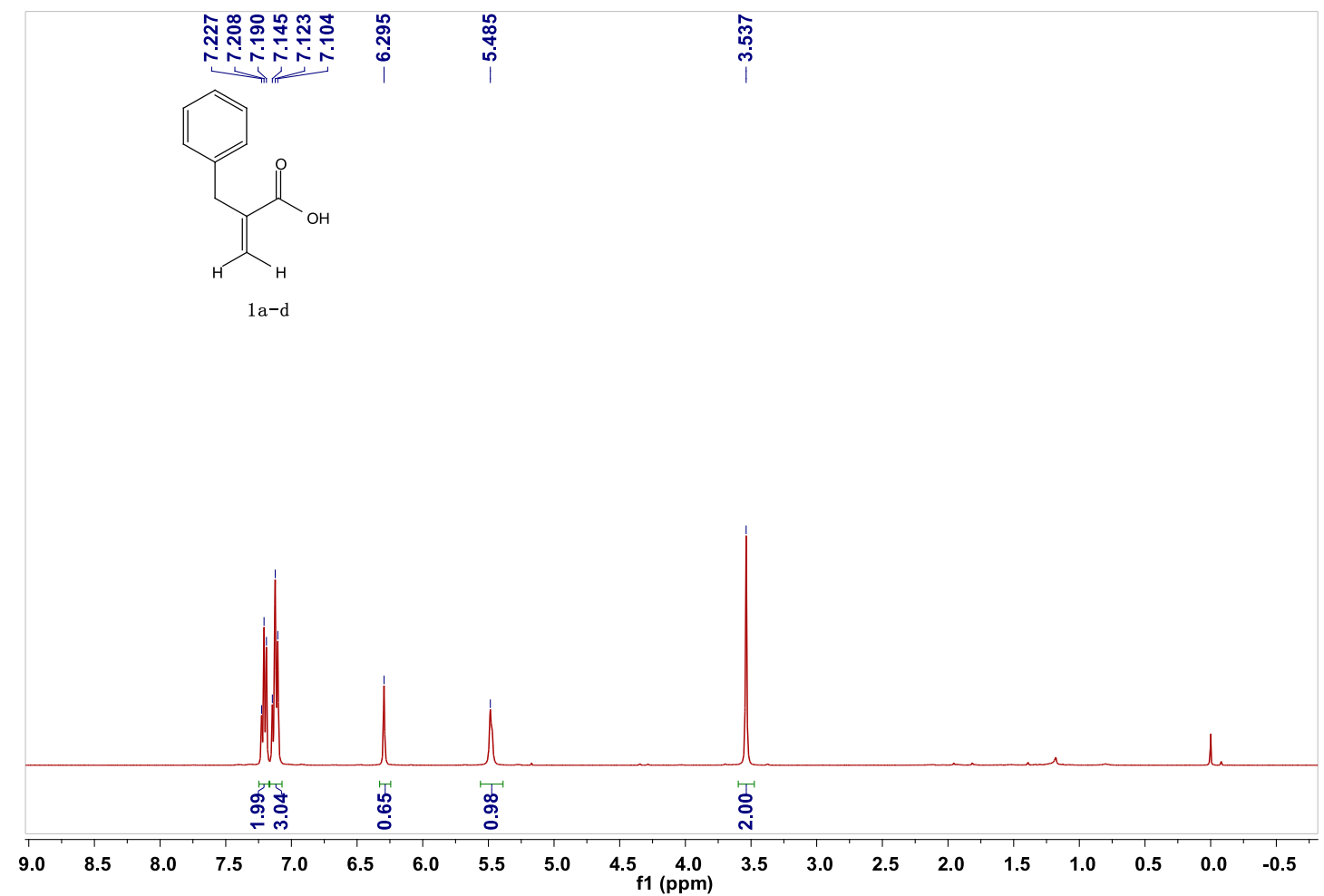



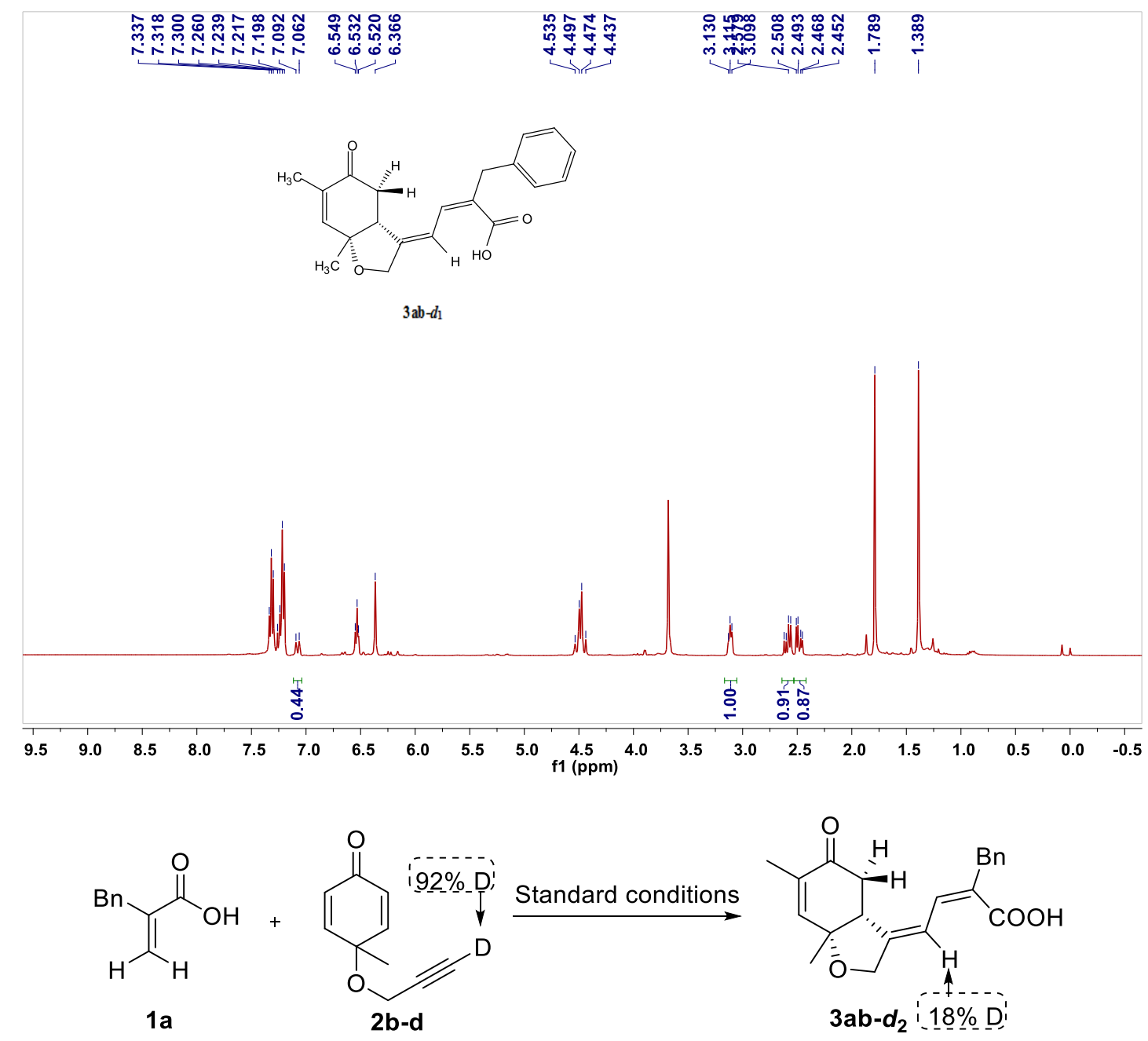

A mixture of $\mathbf{1 a}\left(0.6 \mathrm{mmol}, 3\right.$ equiv), $\mathbf{2 b}-\boldsymbol{d}(0.2 \mathrm{mmol})$, and $\mathrm{Cp} * \mathrm{Rh}(\mathrm{OAc})_{2} \cdot \mathrm{H}_{2} \mathrm{O}(0.016$ mmol, $8.0 \mathrm{~mol} \%)$ were added into a reaction tube. DCE $(6.0 \mathrm{~mL})$ was added and the mixture was stirred at $60{ }^{\circ} \mathrm{C}$ by oil bath for $3 \mathrm{~h}$. Then the solvent was evaporated under reduced pressure and the residue was adsorbed onto small amounts of silica. The product 3ab- $\boldsymbol{d}_{\mathbf{2}}$ were obtained by flash column chromatography on silica gel (eluent: $\mathrm{PE} / \mathrm{EA}=6: 1$ to $2: 1)$. 


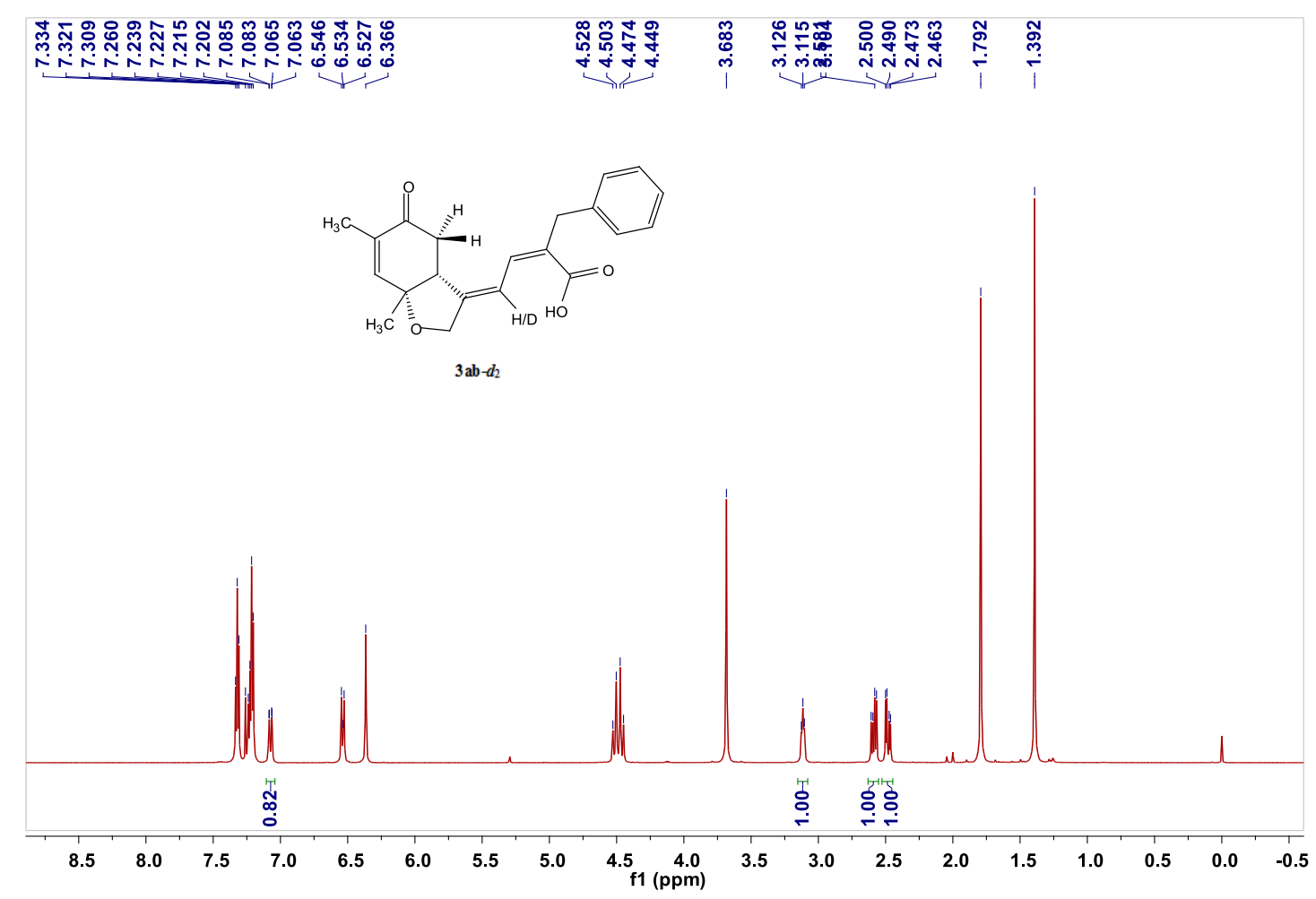

KIE experiments:

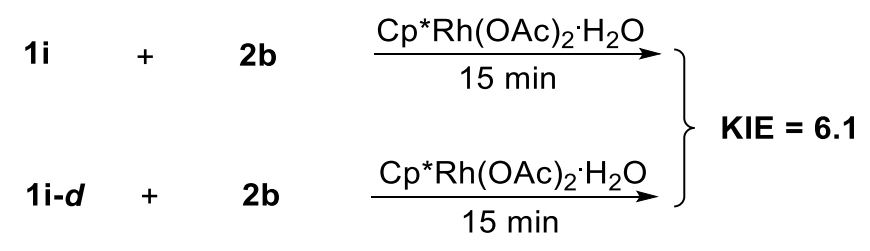

Two tubes each was charged with $\mathbf{1 i}(0.3 \mathrm{mmol}, 3$ equiv) or $\mathbf{1 i}-\boldsymbol{d}(0.3 \mathrm{mmol}, 3$ equiv). To each tube was added $\mathbf{2 b}(0.1 \mathrm{mmol}, 1 \mathrm{eq}), \mathrm{Cp} * \mathrm{Rh}(\mathrm{OAc})_{2} \mathrm{H}_{2} \mathrm{O}(0.008 \mathrm{mmol}, 8.0$ mol \%) and DCE (3 mL). Then the mixture was stirred at $60{ }^{\circ} \mathrm{C}$ by oil bath for 15 minutes. The two reaction tubes were quenched with $n$-pentane and the reaction mixtures were rapidly combined. The solvent was rapidly evaporated under reduced pressure. After the solvent was removed under reduced pressure, the residue was purified by silica gel chromatography using PE/EA (2:1) to afford 3ib and 3ib-d. The KIE value was determined to be $k_{\mathrm{H}} / k_{\mathrm{D}}=6.1$ on the basis of ${ }^{1} \mathrm{H}$ NMR analysis. 


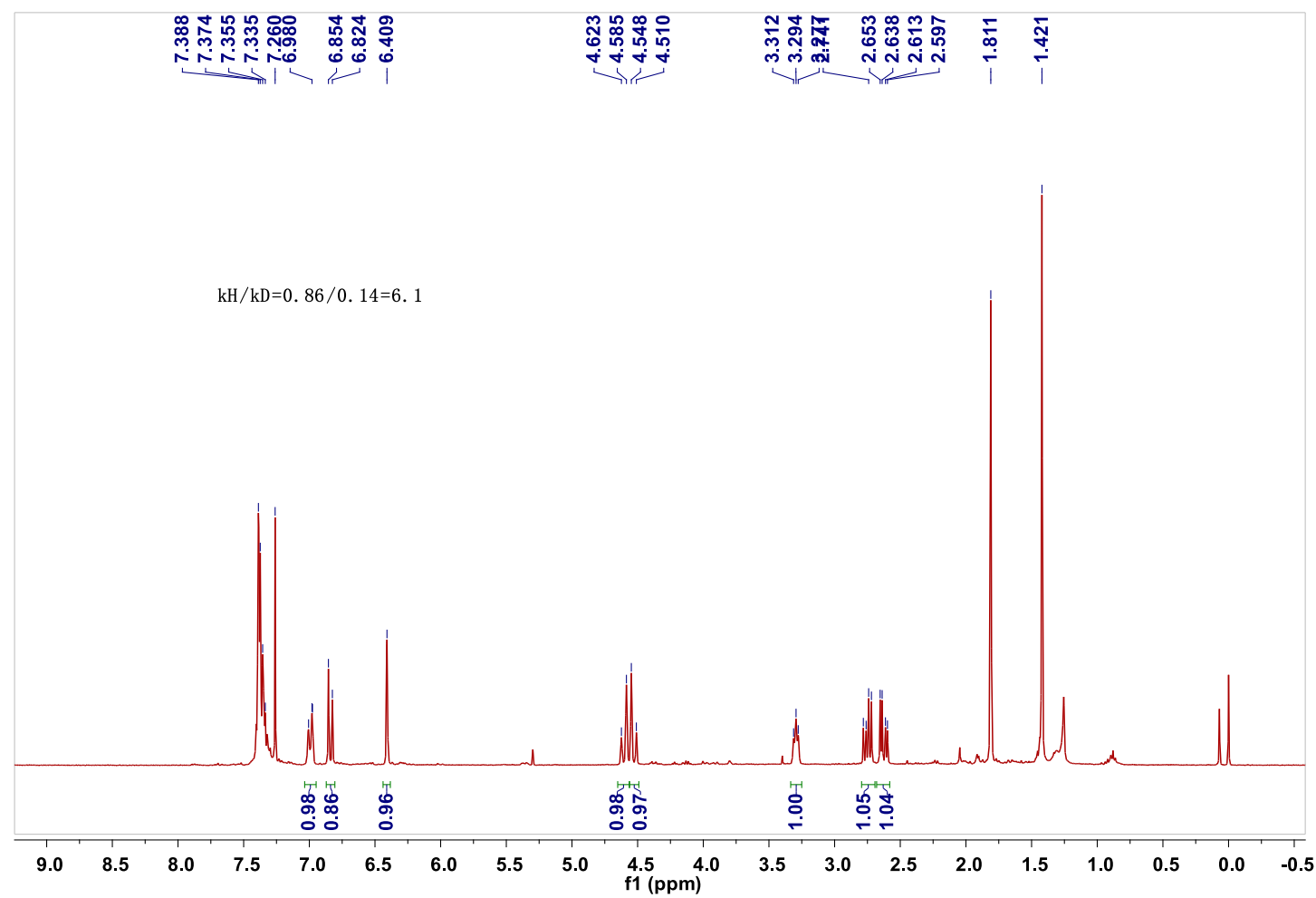

\section{Derivatization reactions}

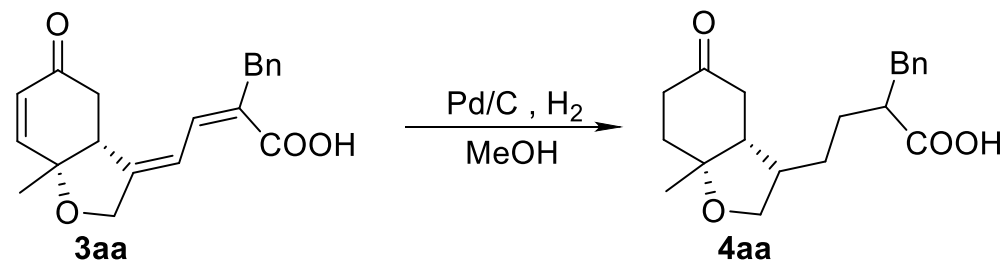

Compound 3aa $(0.1 \mathrm{mmol})$ was dissolved in $2 \mathrm{~mL} \mathrm{MeOH}$ and $10 \% \mathrm{Pd} / \mathrm{C}(0.015 \mathrm{mmol}$, 15 mol \%) was added. The reaction vessel was evacuated under gentle vacuum and backfilled with $\mathrm{H}_{2}$ (balloon) three times. The mixture was stirred at room temperature for 2.5 hours during which time TLC indicated completed conversion. The mixture was filtered through a short plug of silica gel and eluted with fresh EtOAc. The filtrate was concentrated in vacuo, and the crude product was purified by silica gel chromatography using PE/EA (2:1) to afford the product 4aa in $72 \%$ yield.

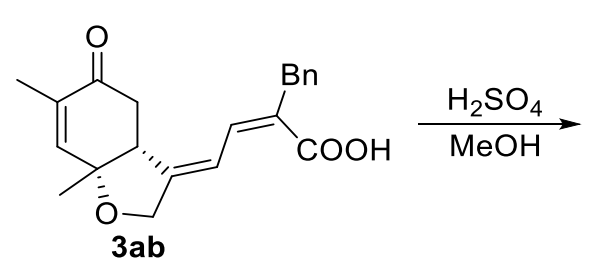<smiles>COC(=O)/C=C(\CO)c1cc(O)c(C)cc1C</smiles>

To a mixture of $\mathbf{3 a b}(0.2 \mathrm{mmol})$ and $\mathrm{MeOH}(5 \mathrm{~mL})$ was added one drop of conc. $\mathrm{H}_{2} \mathrm{SO}_{4}$. The reaction mixture was stirred at room temperature for $40 \mathrm{~h}$. Followed by 
quenching with $\mathrm{H}_{2} \mathrm{O}$, and the residue was extracted with EtOAc. The organic phase was combined, dried with $\mathrm{Na}_{2} \mathrm{SO}_{4}$, and evaporated under reduced pressure. The residue was purified by silica gel chromatography to afford the product $\mathbf{5 a b}$ in $99 \%$ yield.<smiles>CC1=C[C@]2(C)OC/C(=C/C=C(\Cc3ccccc3)C(=O)O)[C@H]2CC1=O</smiles>

$3 a b$<smiles>COC(=O)/C(=C\C=C1/CO[C@]2(C)C=C(C)C(=O)C[C@H]12)Cc1ccccc1</smiles>

6ab

A mixture of 3ab ( 1 mmol, 1 equiv) with $\mathrm{K}_{2} \mathrm{CO}_{3}$ ( 3 mmol, 3 equiv), MeI (6 mmol, 6 equiv), and DMF (5 mL) was stirred at room temperature for $10 \mathrm{~h}$. Followed by quenching with $\mathrm{H}_{2} \mathrm{O}$, and the residue was extracted with EtOAc. The organic phase was combined, dried with $\mathrm{Na}_{2} \mathrm{SO}_{4}$ and evaporated under reduced pressure to afford the product 6ab in $99 \%$ yield.<smiles>COC(=O)/C=C/C=C1/CO[C@]2(C)C=C(C)C(=O)C[C@H]12</smiles>

$6 a b$

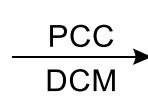<smiles>COC(=O)/C=C(\Cc1ccccc1)C1C(=O)O[C@]2(C)C=C(C)C(=O)C[C@H]12</smiles>

$7 \mathrm{ab}$

A mixture of $6 \mathbf{a b}(0.2 \mathrm{mmol})$ and PCC $(0.8 \mathrm{mmol})$ in DCM $(2 \mathrm{~mL})$ was stirred at $70{ }^{\circ} \mathrm{C}$ by oil bath for $24 \mathrm{~h}$. Then the mixture was concentrated under reduced pressure. The residue was purified by flash column chromatography using PE/EA to afford the product 7ab in $63 \%$ yield. 
Antitumor Bio-activities of Selected Compounds
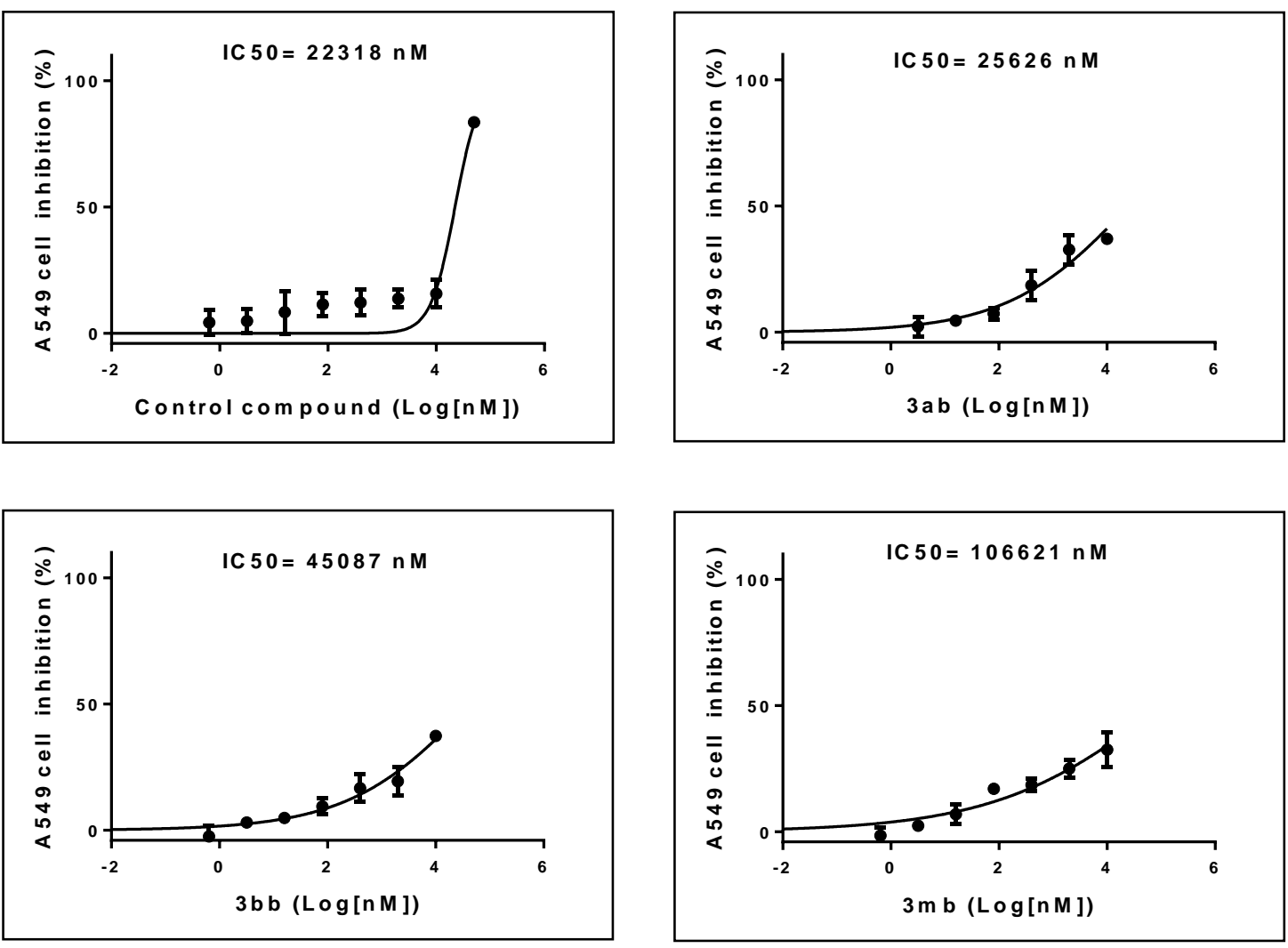


\section{Characterization Data}<smiles>CC12C=CC(=O)C[C@H]1/C(=C\C=C(\Cc1ccccc1)C(=O)O)CO2</smiles>

(Z)-2-benzyl-4-((E)-7a-methyl-5-oxo-3a,4,5,7a-tetrahydrobenzofuran-3(2H)ylidene)but-2-enoic acid (3aa).

Yellow solid (55 mg, 85\%, m.p. $61-63{ }^{\circ} \mathrm{C}$, eluent: PE/EA = 2:1). ${ }^{1} \mathrm{H}$ NMR $(600 \mathrm{MHz}$, $\left.\mathrm{CDCl}_{3}\right) \delta 7.32(\mathrm{t}, J=7.5 \mathrm{~Hz}, 2 \mathrm{H}), 7.24-7.20(\mathrm{~m}, 3 \mathrm{H}), 7.09(\mathrm{dd}, J=11.9,1.7 \mathrm{~Hz}, 1 \mathrm{H})$, $6.59(\mathrm{~d}, J=10.2 \mathrm{~Hz}, 1 \mathrm{H}), 6.52(\mathrm{~d}, J=11.9 \mathrm{~Hz}, 1 \mathrm{H}), 6.03(\mathrm{~d}, J=10.2 \mathrm{~Hz}, 1 \mathrm{H}), 4.52-$ $4.43(\mathrm{~m}, 2 \mathrm{H}), 3.68(\mathrm{~s}, 2 \mathrm{H}), 3.14-3.12(\mathrm{~m}, 1 \mathrm{H}), 2.59(\mathrm{dd}, J=16.4,7.3 \mathrm{~Hz}, 1 \mathrm{H}), 2.50$ $(\mathrm{dd}, J=16.4,6.1 \mathrm{~Hz}, 1 \mathrm{H}), 1.43(\mathrm{~s}, 3 \mathrm{H}) .{ }^{13} \mathrm{C} \mathrm{NMR}\left(100 \mathrm{MHz}, \mathrm{CDCl}_{3}\right) \delta 197.1,171.8$, $150.8,149.5,138.9,137.4,129.1,128.8,126.7,118.8,79.6,70.9,46.0,40.2,39.4,24.4$. HRMS (ESI-TOF) m/z: [M + Na ${ }^{+}$Calcd for $\mathrm{C}_{20} \mathrm{H}_{20} \mathrm{NaO}_{4}{ }^{+}$347.1254, Found: 347.1248.<smiles>CC1=C[C@]2(C)OC/C(=C/C=C(\Cc3ccccc3)C(=O)O)[C@H]2CC1=O</smiles>

(Z)-2-benzyl-4-((E)-6,7a-dimethyl-5-oxo-3a,4,5,7a-tetrahydrobenzofuran-3(2H)ylidene)but-2-enoic acid (3ab).

Yellow solid (62 mg, 91\%, m.p. $43-45^{\circ} \mathrm{C}$, eluent: PE/EA = 2:1). ${ }^{1} \mathrm{H}$ NMR $(600 \mathrm{MHz}$, $\left.\mathrm{CDCl}_{3}\right) \delta 7.32(\mathrm{t}, J=7.5 \mathrm{~Hz}, 2 \mathrm{H}), 7.24-7.20(\mathrm{~m}, 3 \mathrm{H}), 7.07(\mathrm{dd}, J=11.9,1.6 \mathrm{~Hz}, 1 \mathrm{H})$, $6.54(\mathrm{~d}, J=11.9 \mathrm{~Hz}, 1 \mathrm{H}), 6.37(\mathrm{~d}, J=0.9 \mathrm{~Hz}, 1 \mathrm{H}), 4.51(\mathrm{~d}, J=15.0 \mathrm{~Hz}, 1 \mathrm{H}), 4.46(\mathrm{~d}$, $J=15.1 \mathrm{~Hz}, 1 \mathrm{H}), 3.68(\mathrm{~s}, 2 \mathrm{H}), 3.12-3.10(\mathrm{~m}, 1 \mathrm{H}), 1.79(\mathrm{~s}, 3 \mathrm{H}), 1.39(\mathrm{~s}, 3 \mathrm{H}) .{ }^{13} \mathrm{C} \mathrm{NMR}$ $\left(150 \mathrm{MHz}, \mathrm{CDCl}_{3}\right) \delta 197.6,172.2,151.5,144.6,139.0,137.8,136.6,129.4,129.0$, 128.7, 126.7, 118.6, 80.1, 70.7, 46.2, 40.2, 39.6, 24.8, 15.8. HRMS (ESI-TOF) m/z: [M $+\mathrm{Na}]^{+}$Calcd for Chemical Formula: $\mathrm{C}_{21} \mathrm{H}_{22} \mathrm{NaO}_{4}{ }^{+}$361.1410, Found: 361.1414 .<smiles>CC(C)CC1=CC2(C)OC/C(=C/C=C(\Cc3ccccc3)C(=O)O)[C@H]2CC1=O</smiles> 
tetrahydrobenzofuran-3(2H)-ylidene)but-2-enoic acid (3ac).

Yellow solid (60 mg, 79\%, m.p. $184-186{ }^{\circ} \mathrm{C}$, eluent: PE/EA = 2:1). ${ }^{1} \mathrm{H}$ NMR (400 $\left.\mathrm{MHz}, \mathrm{CDCl}_{3}\right) \delta 7.32(\mathrm{t}, J=7.4 \mathrm{~Hz}, 2 \mathrm{H}), 7.24-7.20(\mathrm{~m}, 3 \mathrm{H}), 7.10-7.07$ (m, 1H), 6.56 $(\mathrm{d}, J=12.0 \mathrm{~Hz}, 1 \mathrm{H}), 6.32(\mathrm{~s}, 1 \mathrm{H}), 4.53-4.44(\mathrm{~m}, 2 \mathrm{H}), 3.68(\mathrm{~s}, 2 \mathrm{H}), 3.13-3.10(\mathrm{~m}$, 1H), $2.56(\mathrm{dd}, J=15.0,8.8 \mathrm{~Hz}, 1 \mathrm{H}), 2.42(\mathrm{dd}, J=15.0,5.8 \mathrm{~Hz}, 1 \mathrm{H}), 1.39$ (s, 3H), 1.17 (s, 9H). ${ }^{13} \mathrm{C}$ NMR $\left(100 \mathrm{MHz}, \mathrm{CDCl}_{3}\right) \delta 197.8,172.4,151.6,147.5,141.3,139.0,137.9$, 129.3, 129.0, 128.7, 126.6, 118.6, 80.3, 70.6, 45.6, 42.0, 40.2, 34.6, 29.3, 25.2. HRMS (ESI-TOF) m/z: [M + Na] $]^{+}$Calcd for $\mathrm{C}_{24} \mathrm{H}_{28} \mathrm{NaO}_{4}{ }^{+}$403.1880, Found: 403.1870 .<smiles>CC12C=C(Br)C(=O)C[C@H]1/C(=C\C=C(\Cc1ccccc1)C(=O)O)CO2</smiles>

(Z)-2-benzyl-4-((E)-6-bromo-7a-methyl-5-oxo-3a,4,5,7a-tetrahydrobenzofuran3(2H)-ylidene)but-2-enoic acid (3ad).

White solid (70 mg, 87\%, m.p. $79-81{ }^{\circ} \mathrm{C}$, eluent: PE/EA = 2:1). ${ }^{1} \mathrm{H}$ NMR (600 MHz, $\left.\mathrm{CDCl}_{3}\right) \delta 7.33(\mathrm{t}, J=7.6 \mathrm{~Hz}, 2 \mathrm{H}), 7.25-7.19(\mathrm{~m}, 3 \mathrm{H}), 7.09(\mathrm{dd}, J=11.9,1.8 \mathrm{~Hz}, 1 \mathrm{H})$, $7.03(\mathrm{~s}, 1 \mathrm{H}), 6.46(\mathrm{~d}, J=11.9 \mathrm{~Hz}, 1 \mathrm{H}), 4.58-4.41(\mathrm{~m}, 2 \mathrm{H}), 3.74-3.63(\mathrm{~m}, 2 \mathrm{H}), 3.15$ $-3.13(\mathrm{~m}, J=6.0 \mathrm{~Hz}, 1 \mathrm{H}), 2.77(\mathrm{dd}, J=16.3,7.1 \mathrm{~Hz}, 1 \mathrm{H}), 2.63(\mathrm{dd}, J=16.3,5.8 \mathrm{~Hz}$, 1H), $1.46(\mathrm{~s}, 3 \mathrm{H}) .{ }^{13} \mathrm{C} \mathrm{NMR}\left(150 \mathrm{MHz}, \mathrm{CDCl}_{3}\right) \delta 188.9,172.1,150.0,149.3,138.7$, $137.0,130.3,129.2,128.8,126.8,125.0,119.3,82.2,71.1,46.1,40.1,39.3,24.2$. HRMS (ESI-TOF) m/z: $[\mathrm{M}+\mathrm{Na}]{ }^{+}$Calcd for $\mathrm{C}_{20} \mathrm{H}_{19} \mathrm{BrNaO}_{4}{ }^{+} 425.0359$, Found: 425.0354 .<smiles>CC1=CC(=O)C[C@H](/C=C\C=C(\Cc2ccccc2)C(=O)O)CO1</smiles>

(Z)-2-benzyl-4-((E)-7,7a-dimethyl-5-oxo-3a,4,5,7a-tetrahydrobenzofuran-3(2H)ylidene)but-2-enoic acid (3ae).

Yellow solid (57 mg, 85\%, m.p. $49-51^{\circ} \mathrm{C}$, eluent: PE/EA = 2:1). ${ }^{1} \mathrm{H}$ NMR (600 MHz, $\left.\mathrm{CDCl}_{3}\right) \delta 7.32(\mathrm{t}, J=7.5 \mathrm{~Hz}, 2 \mathrm{H}), 7.23-7.20(\mathrm{~m}, 3 \mathrm{H}), 7.09-7.03(\mathrm{~m}, 1 \mathrm{H}), 6.57$ (d, $J$ $=11.9 \mathrm{~Hz}, 1 \mathrm{H}), 5.91(\mathrm{~s}, 1 \mathrm{H}), 4.42(\mathrm{~d}, J=14.9 \mathrm{~Hz}, 1 \mathrm{H}), 4.30(\mathrm{~d}, J=14.8 \mathrm{~Hz}, 1 \mathrm{H}), 3.68$ 
(s, 2H), $3.10(\mathrm{~m}, 1 \mathrm{H}), 2.65(\mathrm{dd}, J=16.6,6.0 \mathrm{~Hz}, 1 \mathrm{H}), 2.52(\mathrm{dd}, J=16.6,6.0 \mathrm{~Hz}, 1 \mathrm{H})$, $1.97(\mathrm{~s}, 3 \mathrm{H}), 1.46(\mathrm{~s}, 3 \mathrm{H}) .{ }^{13} \mathrm{C} \mathrm{NMR}\left(150 \mathrm{MHz}, \mathrm{CDCl}_{3}\right) \delta 196.4,172.2,160.3,150.3$, 138.9, 137.1, 129.6, 129.1, 128.7, 128.4, 126.6, 118.8, 82.3, 70.7, 47.1, 40.2, 39.1, 22.7, 18.5. HRMS (ESI-TOF) m/z: $[\mathrm{M}+\mathrm{Na}]^{+}$Calcd for $\mathrm{C}_{21} \mathrm{H}_{22} \mathrm{NaO}_{4}{ }^{+}$361.1410, Found: 361.1393.<smiles>CC12OC/C(=C/C=C(\Cc3ccccc3)C(=O)O)[C@H]1CC(=O)C=C2F</smiles>

(Z)-2-benzyl-4-((E)-7-fluoro-7a-methyl-5-oxo-3a,4,5,7a-tetrahydrobenzofuran3(2H)-ylidene)but-2-enoic acid (3af).

White solid (51 mg, 75\%, m.p. $145-147^{\circ} \mathrm{C}$, eluent: PE/EA = 2:1). ${ }^{1} \mathrm{H}$ NMR (600 MHz, $\left.\mathrm{CDCl}_{3}\right) \delta 7.32(\mathrm{t}, J=7.6 \mathrm{~Hz}, 2 \mathrm{H}), 7.25-7.19(\mathrm{~m}, 3 \mathrm{H}), 7.12(\mathrm{dd}, J=11.9,1.9 \mathrm{~Hz}, 1 \mathrm{H})$, $6.47(\mathrm{~d}, J=11.9 \mathrm{~Hz}, 1 \mathrm{H}), 5.80(\mathrm{~d}, J=13.7 \mathrm{~Hz}, 1 \mathrm{H}), 4.57(\mathrm{~d}, J=15.0 \mathrm{~Hz}, 1 \mathrm{H}), 4.52(\mathrm{~d}$, $J=15.1 \mathrm{~Hz}, 1 \mathrm{H}), 3.69(\mathrm{~s}, 2 \mathrm{H}), 3.17(\mathrm{~m}, 1 \mathrm{H}), 2.57(\mathrm{dd}, J=16.4,7.7 \mathrm{~Hz}, 1 \mathrm{H}), 2.50(\mathrm{dd}$, $J=16.5,6.2 \mathrm{~Hz}, 1 \mathrm{H}), 1.53(\mathrm{~s}, 3 \mathrm{H}) .{ }^{13} \mathrm{C} \mathrm{NMR}\left(150 \mathrm{MHz}, \mathrm{CDCl}_{3}\right) \delta 196.1(\mathrm{~d}, J=11.8$ Hz), 175.1 (d, $J=296.0 \mathrm{~Hz}), 172.0,148.8,138.7,136.9$, 130.4, 129.1, 128.8, 126.8, 119.3, $110.8(\mathrm{~d}, J=17.6 \mathrm{~Hz}), 80.1(\mathrm{~d}, J=21.9 \mathrm{~Hz}), 71.1,45.9(\mathrm{~d}, J=5.5 \mathrm{~Hz}), 40.2$, 39.7, 21.1. ${ }^{19} \mathrm{~F}$ NMR $\left(565 \mathrm{MHz}, \mathrm{CDCl}_{3}\right) \delta-94.61(\mathrm{~d}, J=12.5 \mathrm{~Hz}, 1 \mathrm{~F})$. HRMS (ESITOF) $\mathrm{m} / \mathrm{z}:[\mathrm{M}+\mathrm{Na}]^{+} \mathrm{Calcd}$ for $\mathrm{C}_{20} \mathrm{H}_{19} \mathrm{FNaO}_{4}{ }^{+}$365.1160, Found: 365.1156 .<smiles>CCC12C=CC(=O)C[C@H]1/C(=C\C=C(\Cc1ccccc1)C(=O)O)CO2</smiles>

(Z)-2-benzyl-4-((E)-7a-ethyl-5-oxo-3a,4,5,7a-tetrahydrobenzofuran-3(2H)ylidene)but-2-enoic acid (3ag).

Yellow oil (50 mg, 74\%, eluent: PE/EA = 2:1). ${ }^{1} \mathrm{H} \mathrm{NMR}\left(600 \mathrm{MHz}, \mathrm{CDCl}_{3}\right) \delta 7.32(\mathrm{t}$, $J=7.6 \mathrm{~Hz}, 2 \mathrm{H}), 7.24-7.20(\mathrm{~m}, 3 \mathrm{H}), 7.07(\mathrm{dd}, J=11.9,1.9 \mathrm{~Hz}, 1 \mathrm{H}), 6.62(\mathrm{~d}, J=10.3$ Hz, 1H), 6.53 (d, $J=11.9 \mathrm{~Hz}, 1 \mathrm{H}), 6.09$ (d, $J=10.3 \mathrm{~Hz}, 1 \mathrm{H}), 4.49$ (d, $J=14.9 \mathrm{~Hz}, 1 \mathrm{H})$, $4.43(\mathrm{~d}, J=15.0 \mathrm{~Hz}, 1 \mathrm{H}), 3.69(\mathrm{~s}, 2 \mathrm{H}), 3.20-3.18(\mathrm{~m}, 1 \mathrm{H}), 2.59(\mathrm{dd}, J=16.4,7.2 \mathrm{~Hz}$, 1H), $2.50(\mathrm{dd}, J=16.5,6.3 \mathrm{~Hz}, 1 \mathrm{H}), 1.77-1.70(\mathrm{~m}, 2 \mathrm{H}), 0.97(\mathrm{t}, J=7.5 \mathrm{~Hz}, 3 \mathrm{H}) .{ }^{13} \mathrm{C}$ 
NMR (150 MHz, $\left.\mathrm{CDCl}_{3}\right) \delta 197.2,171.9,151.4,148.4,138.9,137.6,130.7,129.5,129.1$, 128.8, 126.7, 118.6, 82.2, 70.8, 43.6, 40.3, 39.8, 3.48, 8.3. HRMS (ESI-TOF) m/z: [M $+\mathrm{Na}]^{+}$Calcd for $\mathrm{C}_{21} \mathrm{H}_{22} \mathrm{NaO}_{4}{ }^{+}$361.1410, Found: 361.1409 .<smiles>CCCCC1(C(C)(C)C)C=CC(=O)C[C@H]1/C=C/C=C(\Cc1ccccc1)C(=O)O</smiles>

(Z)-2-benzyl-4-((E)-7a-butyl-5-oxo-3a,4,5,7a-tetrahydrobenzofuran-3(2H)ylidene)but-2-enoic acid (3ah).

Yellow solid (59 mg, 88\%, m.p. $132-134{ }^{\circ} \mathrm{C}$, eluent: PE/EA = 2:1). ${ }^{1} \mathrm{H}$ NMR (400 $\left.\mathrm{MHz}, \mathrm{CDCl}_{3}\right) \delta 7.31(\mathrm{t}, J=7.3 \mathrm{~Hz}, 2 \mathrm{H}), 7.24-7.18(\mathrm{~m}, 3 \mathrm{H}), 7.11(\mathrm{~d}, J=11.9 \mathrm{~Hz}, 1 \mathrm{H})$, $6.92(\mathrm{~d}, J=10.5 \mathrm{~Hz}, 1 \mathrm{H}), 6.48(\mathrm{~d}, J=11.8 \mathrm{~Hz}, 1 \mathrm{H}), 6.08(\mathrm{~d}, J=10.5 \mathrm{~Hz}, 1 \mathrm{H}), 4.81(\mathrm{~d}$, $J=15.0 \mathrm{~Hz}, 1 \mathrm{H}), 4.58(\mathrm{~d}, J=15.0 \mathrm{~Hz}, 1 \mathrm{H}), 3.73-3.64(\mathrm{~m}, 2 \mathrm{H}), 3.62-3.57$ (m, 2H), $3.39(\mathrm{dd}, J=12.3,6.5 \mathrm{~Hz}, 1 \mathrm{H}), 2.52-2.32(\mathrm{~m}, 2 \mathrm{H}), 1.57-1.46(\mathrm{~m}, 2 \mathrm{H}), 1.39-1.28$ (m, 2H), 0.91 (t, $J=7.3 \mathrm{~Hz}, 3 \mathrm{H}) .{ }^{13} \mathrm{C} \mathrm{NMR}\left(150 \mathrm{MHz}, \mathrm{CDCl}_{3}\right) \delta 197.1,172.3,150.6$, $140.6,138.9,138.5,130.2,129.3,128.9,128.7,126.7,118.1,103.2,77.4,71.4,61.4$, 47.0, 41.1, 40.2, 32.2, 19.5, 14.0. HRMS (ESI-TOF) m/z: $[\mathrm{M}+\mathrm{Na}]^{+}$Calcd for $\mathrm{C}_{23} \mathrm{H}_{26} \mathrm{NaO}_{4}^{+}$389.1723, Found: 389.1729 .<smiles>CCCCCCC12C=CC(=O)C[C@H]1/C(=C\C=C(\Cc1ccccc1)C(=O)O)CO2</smiles>

(Z)-2-benzyl-4-((E)-5-oxo-7a-pentyl-3a,4,5,7a-tetrahydrobenzofuran-3(2H)ylidene)but-2-enoic acid (3ai).

Yellow oil (61 mg, 80\%, eluent: PE/EA = 2:1). ${ }^{1} \mathrm{H} \mathrm{NMR}\left(600 \mathrm{MHz}, \mathrm{CDCl}_{3}\right) \delta 7.32(\mathrm{t}$, $J=7.6 \mathrm{~Hz}, 2 \mathrm{H}), 7.25-7.18(\mathrm{~m}, 3 \mathrm{H}), 7.07(\mathrm{~d}, J=11.9 \mathrm{~Hz}, 1 \mathrm{H}), 6.62(\mathrm{~d}, J=10.3 \mathrm{~Hz}$, 1H), $6.53(\mathrm{~d}, J=11.9 \mathrm{~Hz}, 1 \mathrm{H}), 6.07$ (d, $J=10.3 \mathrm{~Hz}, 1 \mathrm{H}), 4.47(\mathrm{~d}, J=14.9 \mathrm{~Hz}, 1 \mathrm{H})$, $4.42(\mathrm{~d}, J=15.0 \mathrm{~Hz}, 1 \mathrm{H}), 3.69(\mathrm{~s}, 2 \mathrm{H}), 3.20-3.18(\mathrm{~m}, 1 \mathrm{H}), 2.59(\mathrm{dd}, J=16.5,6.9 \mathrm{~Hz}$, $1 \mathrm{H}), 2.50(\mathrm{dd}, J=16.5,6.3 \mathrm{~Hz}, 1 \mathrm{H}), 1.70-1.68(\mathrm{~m}, 2 \mathrm{H}), 1.42-1.23(\mathrm{~m}, 8 \mathrm{H}), 0.89(\mathrm{t}$, $J=6.9 \mathrm{~Hz}, 3 \mathrm{H}) .{ }^{13} \mathrm{C} \mathrm{NMR}\left(150 \mathrm{MHz}, \mathrm{CDCl}_{3}\right) \delta 197.2,172.2,151.2,148.8,139.0,137.5$, 130.4, 129.6, 129.0, 128.7, 126.7, 118.6, 82.0, 77.4, 70.7, 44.1, 40.2, 39.7, 37.8, 31.8, 
29.7, 23.9, 22.7, 14.2. HRMS (ESI-TOF) $\mathrm{m} / \mathrm{z}:[\mathrm{M}+\mathrm{Na}]^{+}$Calcd for $\mathrm{C}_{25} \mathrm{H}_{30} \mathrm{NaO}_{4}{ }^{+}$ 417.2036, Found:417.2041.<smiles>CCCC12C=CC(=O)C[C@H]1/C(=C\C=C(\Cc1ccccc1)C(=O)O)CO2</smiles>

(Z)-2-benzyl-4-((E)-7a-isopropyl-5-oxo-3a,4,5,7a-tetrahydrobenzofuran-3(2H)ylidene)but-2-enoic acid (3aj).

Yellow oil (43 mg, 61\%, eluent: PE/EA = 2:1). ${ }^{1} \mathrm{H} \mathrm{NMR}\left(600 \mathrm{MHz}, \mathrm{CDCl}_{3}\right) \delta 7.32(\mathrm{t}$, $J=7.5 \mathrm{~Hz}, 2 \mathrm{H}), 7.24-7.20(\mathrm{~m}, 3 \mathrm{H}), 7.05(\mathrm{~d}, J=11.8 \mathrm{~Hz}, 1 \mathrm{H}), 6.61(\mathrm{~d}, J=10.4 \mathrm{~Hz}$, $1 \mathrm{H}), 6.52(\mathrm{~d}, J=11.9 \mathrm{~Hz}, 1 \mathrm{H}), 6.16(\mathrm{~d}, J=10.4 \mathrm{~Hz}, 1 \mathrm{H}), 4.43(\mathrm{~d}, J=14.7 \mathrm{~Hz}, 1 \mathrm{H})$, $4.39(\mathrm{~d}, J=14.7 \mathrm{~Hz}, 1 \mathrm{H}), 3.74-3.64(\mathrm{~m}, 2 \mathrm{H}), 3.30-3.27(\mathrm{~m}, 1 \mathrm{H}), 2.58(\mathrm{dd}, J=16.6$, $6.3 \mathrm{~Hz}, 1 \mathrm{H}), 2.52(\mathrm{dd}, J=16.6,6.8 \mathrm{~Hz}, 1 \mathrm{H}), 2.01-1.95(\mathrm{~m}, 1 \mathrm{H}), 1.04(\mathrm{~d}, J=6.9 \mathrm{~Hz}$, $3 \mathrm{H}), 0.93(\mathrm{~d}, J=6.9 \mathrm{~Hz}, 3 \mathrm{H}) .{ }^{13} \mathrm{C} \mathrm{NMR}\left(150 \mathrm{MHz}, \mathrm{CDCl}_{3}\right) \delta 197.4,172.2,152.0,147.0$, 139.0, 137.5, 131.4, 129.6, 129.0, 128.7, 126.7, 118.2, 84.4, 70.4, 41.7, 40.6, 40.3, 34.8, 17.5, 17.0. HRMS (ESI-TOF) m/z: $[\mathrm{M}+\mathrm{Na}]^{+} \mathrm{Calcd}$ for $\mathrm{C}_{22} \mathrm{H}_{24} \mathrm{NaO}_{4}{ }^{+} 375.1567$, Found: 375.1556 .<smiles>O=C1C=C[C@@]2(Cc3ccccc3)OC/C(=C/C=C(\Cc3ccccc3)C(=O)O)[C@H]2C1</smiles>

(Z)-2-benzyl-4-((E)-7a-benzyl-5-oxo-3a,4,5,7a-tetrahydrobenzofuran-3(2H)ylidene)but-2-enoic acid (3ak).

Yellow solid (63 mg, 79\%, m.p. $47-49^{\circ} \mathrm{C}$, eluent: PE/EA = 2:1). ${ }^{1} \mathrm{H}$ NMR $(400 \mathrm{MHz}$, $\left.\mathrm{CDCl}_{3}\right) \delta 7.35-7.27(\mathrm{~m}, 5 \mathrm{H}), 7.25-7.15(\mathrm{~m}, 5 \mathrm{H}), 7.07(\mathrm{~d}, J=11.8 \mathrm{~Hz}, 1 \mathrm{H}), 6.58(\mathrm{~d}$, $J=10.3 \mathrm{~Hz}, 1 \mathrm{H}), 6.48(\mathrm{~d}, J=11.9 \mathrm{~Hz}, 1 \mathrm{H}), 6.05(\mathrm{~d}, J=10.3 \mathrm{~Hz}, 1 \mathrm{H}), 4.55-4.44(\mathrm{~m}$, 2H), $3.75-3.63(\mathrm{~m}, 2 \mathrm{H}), 3.24(\mathrm{~m}, 1 \mathrm{H}), 3.08(\mathrm{~d}, J=13.7 \mathrm{~Hz}, 1 \mathrm{H}), 2.96(\mathrm{~d}, J=13.7 \mathrm{~Hz}$, 1H), $2.51(\mathrm{dd}, J=16.5,6.5 \mathrm{~Hz}, 1 \mathrm{H}), 2.23(\mathrm{dd}, J=16.5,6.2 \mathrm{~Hz}, 1 \mathrm{H}) \cdot{ }^{13} \mathrm{C}$ NMR $(150$ $\left.\mathrm{MHz}, \mathrm{CDCl}_{3}\right) \delta 197.1,172.2,150.5,148.4,138.9,137.4,135.3,130.6,130.3,129.7$, 129.1, 128.7, 128.6, 127.3, 126.6, 118.7, 82.2, 70.9, 44.1, 43.9, 40.1, 39.4. HRMS (ESITOF) $\mathrm{m} / \mathrm{z}:[\mathrm{M}+\mathrm{Na}]^{+}$Calcd for $\mathrm{C}_{26} \mathrm{H}_{24} \mathrm{NaO}_{4}{ }^{+}$423.1567, Found: 423.1569 . 


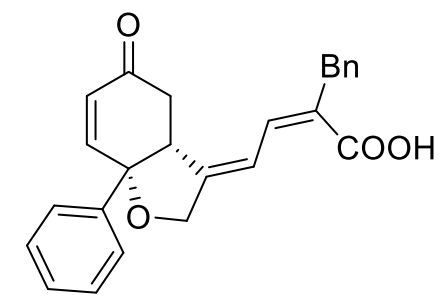

(Z)-2-benzyl-4-((E)-5-oxo-7a-phenyl-3a,4,5,7a-tetrahydrobenzofuran-3(2H)ylidene)but-2-enoic acid (3al).

Yellow solid (71 mg, 92\%, m.p. $76-78{ }^{\circ} \mathrm{C}$, eluent: PE/EA = 2:1). ${ }^{1} \mathrm{H}$ NMR $(600 \mathrm{MHz}$, $\left.\mathrm{CDCl}_{3}\right) \delta 7.44-7.38(\mathrm{~m}, 4 \mathrm{H}), 7.35-7.33(\mathrm{~m}, 1 \mathrm{H}), 7.30(\mathrm{t}, J=7.6 \mathrm{~Hz}, 2 \mathrm{H}), 7.21(\mathrm{t}, J$ $=7.4 \mathrm{~Hz}, 1 \mathrm{H}), 7.17(\mathrm{~d}, J=7.5 \mathrm{~Hz}, 2 \mathrm{H}), 7.05(\mathrm{dd}, J=11.9,1.7 \mathrm{~Hz}, 1 \mathrm{H}), 6.61(\mathrm{~d}, J=$ $10.2 \mathrm{~Hz}, 1 \mathrm{H}), 6.47$ (d, $J=11.9 \mathrm{~Hz}, 1 \mathrm{H}), 6.19(\mathrm{~d}, J=10.2 \mathrm{~Hz}, 1 \mathrm{H}), 4.68-4.59$ (m, 2H), $3.68-3.62(\mathrm{~m}, 2 \mathrm{H}), 3.51-3.49(\mathrm{~m}, 1 \mathrm{H}), 2.66(\mathrm{dd}, J=16.4,6.9 \mathrm{~Hz}, 1 \mathrm{H}), 2.61(\mathrm{dd}, J=$ 16.4, 6.1 Hz, 1H). ${ }^{13} \mathrm{C}$ NMR (150 MHz, $\left.\mathrm{CDCl}_{3}\right) \delta 197.1,172.1,149.9,148.0,141.2$, 138.8, 137.2, 130.1, 129.8, 129.0, 128.7, 128.5, 126.7, 125.4, 118.5, 83.7, 71.5, 47.7, 40.2, 39.3. HRMS (ESI-TOF) m/z: $[\mathrm{M}+\mathrm{Na}]^{+}$Calcd for $\mathrm{C}_{25} \mathrm{H}_{22} \mathrm{NaO}_{4}{ }^{+} 449.1410$, Found: 409.1406.

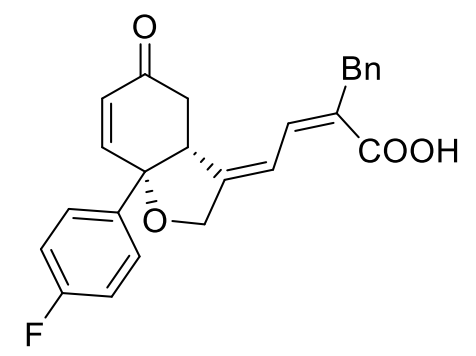

\section{(Z)-2-benzyl-4-((E)-7a-(4-fluorophenyl)-5-oxo-3a,4,5,7a-tetrahydrobenzofuran-}

\section{3(2H)-ylidene)but-2-enoic acid (3am).}

Yellow oil (60 mg, 74\%, eluent: PE/EA = 2:1). ${ }^{1} \mathrm{H} \mathrm{NMR}\left(600 \mathrm{MHz}, \mathrm{CDCl}_{3}\right) \delta 7.39(\mathrm{dd}$, $J=8.6,5.3 \mathrm{~Hz}, 2 \mathrm{H}), 7.30(\mathrm{t}, J=7.5 \mathrm{~Hz}, 2 \mathrm{H}), 7.21(\mathrm{t}, J=7.4 \mathrm{~Hz}, 1 \mathrm{H}), 7.17(\mathrm{~d}, J=7.5$ Hz, 2H), $7.12-7.02$ (m, 3H), $6.58(\mathrm{~d}, J=10.2 \mathrm{~Hz}, 1 \mathrm{H}), 6.46(\mathrm{~d}, J=11.9 \mathrm{~Hz}, 1 \mathrm{H}), 6.19$ $(\mathrm{d}, J=10.2 \mathrm{~Hz}, 1 \mathrm{H}), 4.66-4.57(\mathrm{~m}, 2 \mathrm{H}), 3.66(\mathrm{~s}, 2 \mathrm{H}), 3.45-3.43(\mathrm{~m}, 1 \mathrm{H}), 2.66(\mathrm{dd}$, $J=16.4,6.9 \mathrm{~Hz}, 1 \mathrm{H}), 2.59(\mathrm{dd}, J=16.4,6.0 \mathrm{~Hz}, 1 \mathrm{H}) .{ }^{13} \mathrm{C} \mathrm{NMR}\left(150 \mathrm{MHz}, \mathrm{CDCl}_{3}\right) \delta$ $196.8,171.9,162.8(\mathrm{~d}, J=246.0 \mathrm{~Hz}), 149.5,147.7,138.8,137.1,137.0(\mathrm{~d}, J=2.9 \mathrm{~Hz})$, 130.3, 130.0, 129.1, 128.8, 127.3 (d, $J=7.8 \mathrm{~Hz}), 126.7,118.7,116.0$ (d, $J=21.5 \mathrm{~Hz})$, 83.4, 71.5, 47.9, 40.2, 39.2. ${ }^{19} \mathrm{~F}$ NMR (565 MHz, $\left.\mathrm{CDCl}_{3}\right) \delta-113.6--113.7(\mathrm{~m}, 1 \mathrm{~F})$. 
HRMS (ESI-TOF) m/z: $\left[\mathrm{M}+\mathrm{Na}{ }^{+}\right.$Calcd for $\mathrm{C}_{25} \mathrm{H}_{21} \mathrm{FNaO}_{4}{ }^{+}$427.1316, Found: 427.1315 .

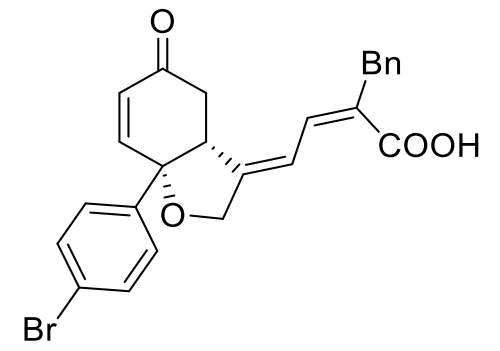

(Z)-2-benzyl-4-((E)-7a-(4-bromophenyl)-5-oxo-3a,4,5,7a-tetrahydrobenzofuran3(2H)-ylidene)but-2-enoic acid (3an).

White solid (85 mg, 91\%, m.p. $76-78{ }^{\circ} \mathrm{C}$, eluent: PE/EA = 2:1). ${ }^{1} \mathrm{H}$ NMR (600 MHz, $\left.\mathrm{CDCl}_{3}\right) \delta 7.52(\mathrm{~d}, J=8.4 \mathrm{~Hz}, 2 \mathrm{H}), 7.31-7.28(\mathrm{~m}, 4 \mathrm{H}), 7.21(\mathrm{t}, J=7.4 \mathrm{~Hz}, 1 \mathrm{H}), 7.17$ $(\mathrm{d}, J=7.6 \mathrm{~Hz}, 2 \mathrm{H}), 7.05(\mathrm{dd}, J=11.8,1.4 \mathrm{~Hz}, 1 \mathrm{H}), 6.56(\mathrm{~d}, J=10.2 \mathrm{~Hz}, 1 \mathrm{H}), 6.44(\mathrm{~d}$, $J=11.9 \mathrm{~Hz}, 1 \mathrm{H}), 6.20(\mathrm{~d}, J=10.2 \mathrm{~Hz}, 1 \mathrm{H}), 4.64(\mathrm{~d}, J=14.9 \mathrm{~Hz}, 1 \mathrm{H}), 4.60$ (d, $J=14.9$ $\mathrm{Hz}, 1 \mathrm{H}), 3.70-3.61(\mathrm{~m}, 2 \mathrm{H}), 3.44-3.42(\mathrm{~m}, 1 \mathrm{H}), 2.64(\mathrm{dd}, J=16.4,7.1 \mathrm{~Hz}, 1 \mathrm{H}), 2.58$ $(\mathrm{dd}, J=16.4,6.0 \mathrm{~Hz}, 1 \mathrm{H}) .{ }^{13} \mathrm{C} \mathrm{NMR}\left(150 \mathrm{MHz}, \mathrm{CDCl}_{3}\right) \delta 196.7,172.0,149.3,147.3$, 140.4, 138.7, 137.0, 132.2, 130.4, 130.1, 129.0, 128.8, 127.2, 126.7, 122.6, 118.7, 83.3, 71.5, 47.7, 40.2, 39.2. HRMS (ESI-TOF) $\mathrm{m} / \mathrm{z}:[\mathrm{M}+\mathrm{Na}]^{+}$Calcd for $\mathrm{C}_{25} \mathrm{H}_{21} \mathrm{BrNaO}_{4}{ }^{+}$ 487.0515, Found: 487.0515 .<smiles></smiles>

(2Z,4E)-2-benzyl-4-(8b-methyl-5-oxo-4,5,6,7,8,8b-hexahydro-2H-indeno[4,5b]furan-3(3aH)-ylidene)but-2-enoic acid (3ao).

Yellow solid (66 mg, 90\%, m.p. $56-58{ }^{\circ} \mathrm{C}$, eluent: PE/EA = 2:1). ${ }^{1} \mathrm{H}$ NMR (400 MHz, $\left.\mathrm{CDCl}_{3}\right) \delta 7.32(\mathrm{t}, J=7.3 \mathrm{~Hz}, 2 \mathrm{H}), 7.24-7.20(\mathrm{~m}, 3 \mathrm{H}), 7.07(\mathrm{~d}, J=11.8 \mathrm{~Hz}, 1 \mathrm{H}), 6.57$ $(\mathrm{d}, J=11.9 \mathrm{~Hz}, 1 \mathrm{H}), 4.45(\mathrm{~d}, J=14.9 \mathrm{~Hz}, 1 \mathrm{H}), 4.38(\mathrm{~d}, J=15.0 \mathrm{~Hz}, 1 \mathrm{H}), 3.68(\mathrm{~s}, 2 \mathrm{H})$, $3.15-3.13(\mathrm{~m}, 1 \mathrm{H}), 2.75-2.47(\mathrm{~m}, 6 \mathrm{H}), 1.99-1.84(\mathrm{~m}, 2 \mathrm{H}), 1.43(\mathrm{~s}, 3 \mathrm{H}) .{ }^{13} \mathrm{C}$ NMR $\left(150 \mathrm{MHz}, \mathrm{CDCl}_{3}\right) \delta 194.8,172.2,162.5,151.2,139.6,139.0,137.6,129.4,129.1$, 128.7, 126.6, 118.7, 80.7, 70.9, 48.0, 40.4, 40.2, 32.9, 29.8, 22.9, 21.8. HRMS (ESITOF) m/z: $[\mathrm{M}+\mathrm{Na}]^{+}$Chemical Formula: $\mathrm{C}_{23} \mathrm{H}_{24} \mathrm{NaO}_{4}{ }^{+}$387.1567, Found: 387.1561 . 
$\overbrace{i=1}^{C}$

(2Z,4E)-2-benzyl-4-(5-oxo-4,5,7,8,9,10-hexahydro-2H-naphtho[8a,1-b]furan3(3aH)-ylidene)but-2-enoic acid (3ap).

White solid (47 mg, 64\%, m.p. $222-223{ }^{\circ} \mathrm{C}$, eluent: PE/EA = 2:1). ${ }^{1} \mathrm{H}$ NMR (400 MHz, DMSO) $\delta 12.56(\mathrm{~s}, 1 \mathrm{H}), 7.28(\mathrm{t}, J=7.5 \mathrm{~Hz}, 2 \mathrm{H}), 7.22-7.15(\mathrm{~m}, 3 \mathrm{H}), 6.90(\mathrm{~d}, J=10.7$ $\mathrm{Hz}, 1 \mathrm{H}), 6.76(\mathrm{~d}, J=11.8 \mathrm{~Hz}, 1 \mathrm{H}), 5.84(\mathrm{~s}, 1 \mathrm{H}), 4.48-4.36(\mathrm{~m}, 2 \mathrm{H}), 3.68-3.58(\mathrm{~m}$, 2H), $3.30-3.26(\mathrm{~m}, 1 \mathrm{H}), 2.48-2.46(\mathrm{~m}, 2 \mathrm{H}), 2.41(\mathrm{dd}, J=13.2,4.0 \mathrm{~Hz}, 1 \mathrm{H}), 2.32(\mathrm{~d}$, $J=12.7 \mathrm{~Hz}, 1 \mathrm{H}), 1.96-1.81(\mathrm{~m}, 2 \mathrm{H}), 1.78-1.70(\mathrm{~m}, 1 \mathrm{H}), 1.60-1.57(\mathrm{~m}, 1 \mathrm{H}), 1.53$ $-1.45(\mathrm{~m}, 1 \mathrm{H}), 1.40-1.30(\mathrm{~m}, 1 \mathrm{H}) .{ }^{13} \mathrm{C} \mathrm{NMR}\left(150 \mathrm{MHz}, \mathrm{CDCl}_{3}\right) \delta 197.4,171.7,161.3$, 151.6, 139.0, 137.9, 129.0, 128.7, 126.7, 124.8, 118.7, 81.2, 70.0, 45.3, 40.3, 39.8, 36.8, 33.2, 27.3, 21.6. HRMS (ESI-TOF) $\mathrm{m} / \mathrm{z}:[\mathrm{M}+\mathrm{Na}]^{+} \mathrm{Calcd}$ for $\mathrm{C}_{23} \mathrm{H}_{24} \mathrm{NaO}_{4}{ }^{+} 387.1567$, Found:387.1556.<smiles>C[C@H]1O[C@]2(C)C=CC(=O)C[C@H]2/C1=C\C=C(\Br)C(=O)O</smiles>

(Z)-2-benzyl-4-((E)-2,7a-dimethyl-5-oxo-3a,4,5,7a-tetrahydrobenzofuran-3(2H)ylidene)but-2-enoic acid (3aq).

Yellow solid (55 mg, 82\%, m.p. $78-80{ }^{\circ} \mathrm{C}$, eluent: PE/EA = 2:1). ${ }^{1} \mathrm{H}$ NMR $(600 \mathrm{MHz}$, $\left.\mathrm{CDCl}_{3}\right) \delta 7.33(\mathrm{t}, J=7.6 \mathrm{~Hz}, 2 \mathrm{H}), 7.25-7.22(\mathrm{~m}, 3 \mathrm{H}), 6.94(\mathrm{dt}, J=11.8,2.4 \mathrm{~Hz}, 1 \mathrm{H})$, $6.53(\mathrm{dd}, J=10.3,0.8 \mathrm{~Hz}, 1 \mathrm{H}), 6.50$ (d, $J=11.8 \mathrm{~Hz}, 1 \mathrm{H}), 6.00$ (d, $J=10.3 \mathrm{~Hz}, 1 \mathrm{H})$, $4.45-4.35(\mathrm{~m}, 1 \mathrm{H}), 3.75-3.65(\mathrm{~m}, 2 \mathrm{H}), 3.15-3.14(\mathrm{~m}, 1 \mathrm{H}), 2.64(\mathrm{dd}, J=16.7,3.9$ $\mathrm{Hz}, 1 \mathrm{H}), 2.56(\mathrm{dd}, J=16.7,6.2 \mathrm{~Hz}, 1 \mathrm{H}), 1.49$ (s, 3H), 1.35 (d, $J=6.2 \mathrm{~Hz}, 3 \mathrm{H}) .{ }^{13} \mathrm{C}$ $\operatorname{NMR}\left(150 \mathrm{MHz}, \mathrm{CDCl}_{3}\right) \delta$ 196.7, 172.1, 154.8, 151.2, 138.9, 137.3, 130.0, 129.6, 129.3, 128.8, 126.7, 119.0, 78.2, 76.7, 47.0, 40.7, 39.6, 24.5, 20.1. HRMS (ESI-TOF) m/z: [M $+\mathrm{Na}]^{+}$Calcd for $\mathrm{C}_{21} \mathrm{H}_{22} \mathrm{NaO}_{4}{ }^{+}$361.1410, Found: 361.1406 . 


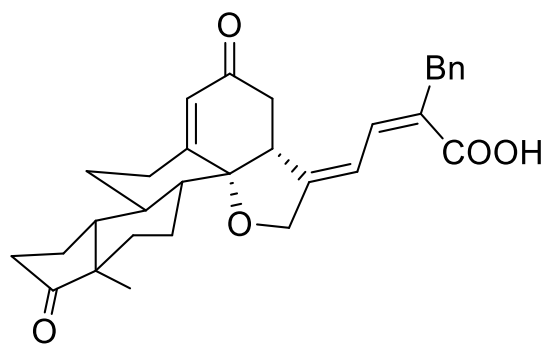

(2Z,4E)-2-benzyl-4-((3aS,5aS,13bS)-3a-methyl-3,10-dioxo-

\section{$1,2,3,3 a, 4,5,5 a, 8 a, 9,10,12,13,13 a, 13 b-$}

tetradecahydrocyclopenta[7,8]phenanthro[4a,4-b]furan-8(7H)-ylidene)but-2enoic acid (3ar).

Yellow solid (49 mg, 50\%, m.p. 96 - $98{ }^{\circ} \mathrm{C}$, eluent: PE/EA = 2:1). ${ }^{1} \mathrm{H}$ NMR $(600 \mathrm{MHz}$, $\left.\mathrm{CDCl}_{3}\right) \delta 7.32(\mathrm{t}, J=7.5 \mathrm{~Hz}, 2 \mathrm{H}), 7.23-7.20(\mathrm{~m}, 3 \mathrm{H}), 7.04(\mathrm{~d}, J=11.5 \mathrm{~Hz}, 1 \mathrm{H}), 6.59$ $(\mathrm{d}, J=11.9 \mathrm{~Hz}, 1 \mathrm{H}), 5.88(\mathrm{~s}, 1 \mathrm{H}), 4.42(\mathrm{~d}, J=14.6 \mathrm{~Hz}, 1 \mathrm{H}), 4.32(\mathrm{~d}, J=14.4 \mathrm{~Hz}, 1 \mathrm{H})$, $3.68(\mathrm{~s}, 2 \mathrm{H}), 3.38(\mathrm{~m}, 1 \mathrm{H}), 2.68(\mathrm{td}, J=13.0,4.5 \mathrm{~Hz}, 1 \mathrm{H}), 2.59(\mathrm{dd}, J=16.2,5.6 \mathrm{~Hz}$, 1H), $2.52-2.43(\mathrm{~m}, 2 \mathrm{H}), 2.27(\mathrm{~d}, J=12.8 \mathrm{~Hz}, 1 \mathrm{H}), 2.14-1.99(\mathrm{~m}, 3 \mathrm{H}), 1.98-1.91$ (m, 1H), $1.87-1.58(\mathrm{~m}, J=13.1 \mathrm{~Hz}, 1 \mathrm{H}), 1.70-1.63(\mathrm{~m}, 2 \mathrm{H}), 1.61-1.54(\mathrm{~m}, 1 \mathrm{H})$, $1.39-1.23(\mathrm{~m}, 3 \mathrm{H}), 1.17-1.10(\mathrm{~m}, 1 \mathrm{H}), 0.93(\mathrm{~s}, 3 \mathrm{H}) .{ }^{13} \mathrm{C} \mathrm{NMR}\left(150 \mathrm{MHz}, \mathrm{CDCl}_{3}\right) \delta$ 196.4, 171.7, 163.4, 150.7, 138.9, 137.0, 129.6, 129.1, 128.7, 126.7, 125.2, 118.2, 84.4, 71.2, 50.8, 50.5, 47.9, 41.3, 40.3, 39.7, 36.1, 35.9, 32.3, 31.5, 31.2, 21.9, 21.3, 13.9. HRMS (ESI-TOF) m/z: [M + Na ${ }^{+}$Calcd for $\mathrm{C}_{31} \mathrm{H}_{34} \mathrm{NaO}_{5}{ }^{+}$509.2298, Found: 509.2297.<smiles>CC1=C[C@]2(C)OC/C(=C/C=C(/C)C(=O)O)[C@H]2CC1=O</smiles>

(Z)-4-((E)-6,7a-dimethyl-5-oxo-3a,4,5,7a-tetrahydrobenzofuran-3(2H)-ylidene)-2methylbut-2-enoic acid (3bb).

Yellow oil (43 mg, 81\%, eluent: PE/EA = 2:1). ${ }^{1} \mathrm{H}$ NMR $\left(600 \mathrm{MHz}, \mathrm{CDCl}_{3}\right) \delta 7.08-$ $7.06(\mathrm{~m}, 1 \mathrm{H}), 6.62(\mathrm{~d}, J=11.9 \mathrm{~Hz}, 1 \mathrm{H}), 6.40(\mathrm{~s}, 1 \mathrm{H}), 4.56(\mathrm{~d}, J=14.9 \mathrm{~Hz}, 1 \mathrm{H}), 4.47$ $(\mathrm{d}, J=15.0 \mathrm{~Hz}, 1 \mathrm{H}), 3.26-3.24(\mathrm{~m}, 1 \mathrm{H}), 2.71(\mathrm{dd}, J=16.1,8.7 \mathrm{~Hz}, 1 \mathrm{H}), 2.61(\mathrm{dd}, J=$ 16.1, $6.1 \mathrm{~Hz}, 1 \mathrm{H}), 2.02(\mathrm{~s}, 3 \mathrm{H}), 1.80(\mathrm{~s}, 3 \mathrm{H}), 1.40(\mathrm{~s}, 3 \mathrm{H}) .{ }^{13} \mathrm{C} \mathrm{NMR}\left(150 \mathrm{MHz}, \mathrm{CDCl}_{3}\right)$ $\delta 198.0,172.7,150.4,144.5,137.0,136.6,125.8,118.8,79.9,70.7,46.0,39.8,25.0$, 
21.0, 15.8. HRMS (ESI-TOF) m/z: $[\mathrm{M}+\mathrm{Na}]^{+} \mathrm{Calcd}$ for $\mathrm{C}_{15} \mathrm{H}_{18} \mathrm{NaO}_{4}{ }^{+}$285.1097, Found: 285.1097.<smiles>CCCCC(=O)C(=CC=C1CO[C@]2(C)C=C(C)C(=O)C[C@@H]12)C(=O)O</smiles>

(Z)-2-(2-((E)-6,7a-dimethyl-5-ox0-3a,4,5,7a-tetrahydrobenzofuran-3(2H)ylidene)ethylidene)pentanoic acid (3cb).

Yellow oil (54 mg, 93\%, eluent: PE/EA = 2:1). ${ }^{1} \mathrm{H}$ NMR $\left(600 \mathrm{MHz}, \mathrm{CDCl}_{3}\right) \delta 7.04-$ $7.01(\mathrm{~m}, 1 \mathrm{H}), 6.56(\mathrm{~d}, J=11.9 \mathrm{~Hz}, 1 \mathrm{H}), 6.40(\mathrm{~d}, J=1.3 \mathrm{~Hz}, 1 \mathrm{H}), 4.55(\mathrm{~d}, J=14.9 \mathrm{~Hz}$, $1 \mathrm{H}), 4.47(\mathrm{~d}, J=15.0 \mathrm{~Hz}, 1 \mathrm{H}), 3.26-3.24(\mathrm{~m}, 1 \mathrm{H}), 2.70(\mathrm{dd}, J=16.1,8.6 \mathrm{~Hz}, 1 \mathrm{H})$, $2.60(\mathrm{dd}, J=16.2,6.1 \mathrm{~Hz}, 1 \mathrm{H}), 2.34-2.28(\mathrm{~m}, 2 \mathrm{H}), 1.80(\mathrm{~d}, J=1.2 \mathrm{~Hz}, 3 \mathrm{H}), 1.54-$ $1.50(\mathrm{~m}, 2 \mathrm{H}), 1.40(\mathrm{~s}, 3 \mathrm{H}), 0.93(\mathrm{t}, J=7.4 \mathrm{~Hz}, 3 \mathrm{H}) .{ }^{13} \mathrm{C} \mathrm{NMR}\left(150 \mathrm{MHz}, \mathrm{CDCl}_{3}\right) \delta$ $197.9,172.8,150.2,144.5,136.5,136.2,130.7,118.8,79.9,70.7,46.0,39.7,36.9,25.0$, 22.8, 15.8, 13.9. HRMS (ESI-TOF) $\mathrm{m} / \mathrm{z}:[\mathrm{M}+\mathrm{Na}]^{+}$Calcd for $\mathrm{C}_{17} \mathrm{H}_{22} \mathrm{NaO}_{4}{ }^{+}$313.1410, Found: 313.1403.<smiles>CCCCC(=CC=C1CO[C@]2(C)C=C(C)C(=O)C[C@@H]12)C(=O)O</smiles>

(Z)-2-(2-((E)-6,7a-dimethyl-5-oxo-3a,4,5,7a-tetrahydrobenzofuran-3(2H)ylidene)ethylidene)hexanoic acid (3db).

Yellow oil (55 mg, 90\%, eluent: PE/EA = 2:1). ${ }^{1} \mathrm{H}$ NMR (400 MHz, $\left.\mathrm{CDCl}_{3}\right) 7.02(\mathrm{dd}$, $J=11.9,1.9 \mathrm{~Hz}, 1 \mathrm{H}), 6.56(\mathrm{~d}, J=11.9 \mathrm{~Hz}, 1 \mathrm{H}), 6.40(\mathrm{~d}, J=1.3 \mathrm{~Hz}, 1 \mathrm{H}), 4.55(\mathrm{~d}, J=$ $14.9 \mathrm{~Hz}, 1 \mathrm{H}), 4.47(\mathrm{~d}, J=15.1 \mathrm{~Hz}, 1 \mathrm{H}), 3.27-3.23(\mathrm{~m}, 1 \mathrm{H}), 2.70(\mathrm{dd}, J=16.1,8.6 \mathrm{~Hz}$, 1H), 2.60 (dd, $J=16.2,6.1 \mathrm{~Hz}, 1 \mathrm{H}), 2.36-2.29(\mathrm{~m}, 2 \mathrm{H}), 1.80$ (d, $J=1.1 \mathrm{~Hz}, 3 \mathrm{H}), 1.50$ - $1.42(\mathrm{~m}, 2 \mathrm{H}), 1.40$ (s, 3H), 1.34 (dd, $J=14.9,7.3 \mathrm{~Hz}, 2 \mathrm{H}), 0.92$ (t, $J=7.3 \mathrm{~Hz}, 1 \mathrm{H})$. ${ }^{13} \mathrm{C}$ NMR $\left(100 \mathrm{MHz}, \mathrm{CDCl}_{3}\right) \delta 197.9,172.9,150.2,144.5,136.5,136.0,130.9,118.8$, 79.9, 70.7 46.0, 39.7, 34.7, 31.7, 25.0, 22.5, 15.8, 14.0. HRMS (ESI-TOF) m/z: [M + $\mathrm{Na}]^{+}$Calcd for $\mathrm{C}_{18} \mathrm{H}_{24} \mathrm{NaO}_{4}{ }^{+}$327.1567, Found: 327.1555 . 
<smiles>CCCC(=CC=C1CO[C@]2(C)C=C(C)C(=O)C[C@@H]12)C(=O)O</smiles>

(Z)-4-((E)-6,7a-dimethyl-5-oxo-3a,4,5,7a-tetrahydrobenzofuran-3(2H)-ylidene)-2isopropylbut-2-enoic acid (3eb).

Yellow oil (53 mg, 91\%, eluent: PE/EA = 2:1). ${ }^{1} \mathrm{H}$ NMR $\left(600 \mathrm{MHz}, \mathrm{CDCl}_{3}\right) \delta 6.92-$ $6.90(\mathrm{~m}, 1 \mathrm{H}), 6.52(\mathrm{~d}, J=11.7 \mathrm{~Hz}, 1 \mathrm{H}), 6.40(\mathrm{~d}, J=1.2 \mathrm{~Hz}, 1 \mathrm{H}), 4.54(\mathrm{~d}, J=14.8 \mathrm{~Hz}$, $1 \mathrm{H}), 4.47(\mathrm{~d}, J=14.8 \mathrm{~Hz}, 1 \mathrm{H}), 3.27-3.25(\mathrm{~m}, 1 \mathrm{H}), 2.88-2.83(\mathrm{~m}, 1 \mathrm{H}), 2.72(\mathrm{dd}, J=$ 16.2, 8.3 Hz, 1H), $2.62(\mathrm{dd}, J=16.2,6.1 \mathrm{~Hz}, 1 \mathrm{H}), 1.81(\mathrm{~d}, J=1.2 \mathrm{~Hz}, 3 \mathrm{H}), 1.42$ (s, 3H), $1.16-1.14(\mathrm{~m}, 6 \mathrm{H}) .{ }^{13} \mathrm{C} \mathrm{NMR}\left(150 \mathrm{MHz}, \mathrm{CDCl}_{3}\right) \delta 197.8,172.7,149.7,144.6,137.3$, 136.6, 131.7, 118.7, 80.0, 70.7, 46.1, 39.6, 31.6, 25.0, 22.3, 22.2, 15.8. HRMS (ESITOF) m/z: $[\mathrm{M}+\mathrm{Na}]^{+}$Calcd for $\mathrm{C}_{17} \mathrm{H}_{22} \mathrm{NaO}_{4}{ }^{+}$313.1410, Found: 313.1402 .<smiles>CC1=C[C@]2(C)OC/C(=C/C=C(\C(=O)O)C(C)(C)C)[C@H]2CC1=O</smiles>

(Z)-2-(2-((E)-6,7a-dimethyl-5-oxo-3a,4,5,7a-tetrahydrobenzofuran-3(2H)ylidene)ethylidene)-4-methylpentanoic acid (3fb).

Yellow oil (52 mg, 85\%, eluent: PE/EA = 2:1). ${ }^{1} \mathrm{H}$ NMR (600 MHz, CDCl3) $87.04-$ $7.01(\mathrm{~m}, 1 \mathrm{H}), 6.53(\mathrm{~d}, J=11.9 \mathrm{~Hz}, 1 \mathrm{H}), 6.40(\mathrm{~m}, 1 \mathrm{H}), 4.56(\mathrm{~d}, J=14.9 \mathrm{~Hz}, 1 \mathrm{H}), 4.48$ $(\mathrm{d}, J=15.0 \mathrm{~Hz}, 1 \mathrm{H}), 3.26-3.24(\mathrm{~m}, 1 \mathrm{H}), 2.71(\mathrm{dd}, J=16.1,8.6 \mathrm{~Hz}, 1 \mathrm{H}), 2.59(\mathrm{dd}, J=$ 16.1, $6.0 \mathrm{~Hz}, 1 \mathrm{H}), 2.26-2.17(\mathrm{~m}, 2 \mathrm{H}), 1.86-1.82(\mathrm{~m}, 1 \mathrm{H}), 1.80(\mathrm{~s}, 3 \mathrm{H}), 1.41(\mathrm{~s}, 3 \mathrm{H})$, $0.94-0.86(\mathrm{~m}, 6 \mathrm{H}) .{ }^{13} \mathrm{C} \mathrm{NMR}\left(150 \mathrm{MHz}, \mathrm{CDCl}_{3}\right) \delta 197.8,173.0,150.3,144.5,137.0$, 136.5, 129.9, 118.7, 79.9, 70.7, 46.0, 44.2, 39.7, 28.2, 24.9, 22.5, 22.4, 15.8. HRMS (ESI-TOF) m/z: [M + Na $]^{+}$Calcd for $\mathrm{C}_{18} \mathrm{H}_{24} \mathrm{NaO}_{4}{ }^{+}$327.1567, Found: 327.1549 .<smiles>C=CC/C(=C/C=C1/CO[C@]2(C)C=C(C)C(=O)C[C@H]12)C(=O)O</smiles>

(Z)-2-(2-((E)-6,7a-dimethyl-5-oxo-3a,4,5,7a-tetrahydrobenzofuran-3(2H)ylidene)ethylidene)pent-4-enoic acid (3gb). 
Yellow oil (44 mg, 76\%, eluent: PE/EA = 2:1). ${ }^{1} \mathrm{H}$ NMR $\left(600 \mathrm{MHz}, \mathrm{CDCl}_{3}\right) \delta 7.09-$ $7.06(\mathrm{~m}, 1 \mathrm{H}), 6.61(\mathrm{~d}, J=11.9 \mathrm{~Hz}, 1 \mathrm{H}), 6.40(\mathrm{~d}, J=1.2 \mathrm{~Hz}, 1 \mathrm{H}), 5.91-5.84(\mathrm{~m}, 1 \mathrm{H})$, $5.15-5.11(\mathrm{~m}, 1 \mathrm{H}), 5.10(\mathrm{~s}, 1 \mathrm{H}), 4.55(\mathrm{~d}, J=15.0 \mathrm{~Hz}, 1 \mathrm{H}), 4.48(\mathrm{~d}, J=15.1 \mathrm{~Hz}, 1 \mathrm{H})$, $3.26-3.24(\mathrm{~m}, 1 \mathrm{H}), 3.15-3.06(\mathrm{~m}, 2 \mathrm{H}), 2.70(\mathrm{dd}, J=16.2,8.4 \mathrm{~Hz}, 1 \mathrm{H}), 2.60(\mathrm{dd}, J=$ 16.2, $6.1 \mathrm{~Hz}, 1 \mathrm{H}), 1.80(\mathrm{~d}, J=1.1 \mathrm{~Hz}, 3 \mathrm{H}), 1.41(\mathrm{~s}, 3 \mathrm{H}) \cdot{ }^{13} \mathrm{C} \mathrm{NMR}\left(150 \mathrm{MHz}, \mathrm{CDCl}_{3}\right)$ $\delta 197.8,172.1,151.3,144.5,137.1,136.6,135.5,128.3,118.6,117.2,80.0,70.7,46.1$, 39.7, 38.3, 24.9, 15.8. HRMS (ESI-TOF) m/z: $[\mathrm{M}+\mathrm{Na}]^{+}$Calcd for $\mathrm{C}_{17} \mathrm{H}_{20} \mathrm{NaO}_{4}{ }^{+}$ 311.1254, Found: 311.1248 .<smiles>CC1=C[C@]2(C)OC/C(=C/C=C(\C(=O)O)c3ccccc3)[C@H]2CC1=O</smiles>

(Z)-4-((E)-6,7a-dimethyl-5-oxo-3a,4,5,7a-tetrahydrobenzofuran-3(2H)-ylidene)-2phenylbut-2-enoic acid (3hb).

Yellow solid (53 mg, 82\%, m.p. $73-75^{\circ} \mathrm{C}$, eluent: PE/EA = 2:1). ${ }^{1} \mathrm{H}$ NMR (600 MHz, $\mathrm{CDCl} 3) \delta 7.43-7.32(\mathrm{~m}, 5 \mathrm{H}), 7.00(\mathrm{~d}, J=12.0,1 \mathrm{H}), 6.85(\mathrm{~d}, J=12.0 \mathrm{~Hz}, 1 \mathrm{H}), 6.41(\mathrm{~s}, 1 \mathrm{H})$, $4.60(\mathrm{~d}, J=15.2 \mathrm{~Hz}, 1 \mathrm{H}), 4.53(\mathrm{~d}, J=15.3 \mathrm{~Hz}, 1 \mathrm{H}), 3.31-3.28(\mathrm{~m}, 1 \mathrm{H}), 2.75(\mathrm{dd}, J=$ 16.2, 8.5 Hz, 1H), $2.63(\mathrm{dd}, J=16.2,6.1 \mathrm{~Hz}, 1 \mathrm{H}), 1.81(\mathrm{~s}, 3 \mathrm{H}), 1.42(\mathrm{~s}, 3 \mathrm{H}) .{ }^{13} \mathrm{C} \mathrm{NMR}$ $\left(150 \mathrm{MHz}, \mathrm{CDCl}_{3}\right) \delta 197.7,172.1,152.6,144.5,138.1,136.6,136.1,131.7,128.5$, 128.4, 128.3, 118.7, 80.0, 70.8, 46.4, 39.7, 24.9, 15.8. HRMS (ESI-TOF) m/z: $[\mathrm{M}+\mathrm{H}]^{+}$ Calcd for $\mathrm{C}_{20} \mathrm{H}_{21} \mathrm{O}_{4}{ }^{+}$325.1434, Found: 325.1433.<smiles>COc1ccc(/C(=C/C=C2/CO[C@]3(C)C=C(C)C(=O)C[C@H]23)C(=O)O)cc1</smiles>

(Z)-4-((E)-6,7a-dimethyl-5-oxo-3a,4,5,7a-tetrahydrobenzofuran-3(2H)-ylidene)-2(4-methoxyphenyl)but-2-enoic acid (3ib).

Yellow solid (55 mg, 78\%, m.p. $76-78{ }^{\circ} \mathrm{C}$, eluent: PE/EA = 2:1). ${ }^{1} \mathrm{H}$ NMR $(600 \mathrm{MHz}$, $\left.\mathrm{CDCl}_{3}\right) \delta 7.33(\mathrm{~d}, J=8.6 \mathrm{~Hz}, 2 \mathrm{H}), 6.94(\mathrm{~d}, J=12.0 \mathrm{~Hz}, 1 \mathrm{H}), 6.90(\mathrm{~d}, J=8.6 \mathrm{~Hz}, 2 \mathrm{H})$, 
$6.78(\mathrm{~d}, J=12.0 \mathrm{~Hz}, 1 \mathrm{H}), 6.41(\mathrm{~s}, 1 \mathrm{H}), 4.59(\mathrm{~d}, J=15.1 \mathrm{~Hz}, 1 \mathrm{H}), 4.52(\mathrm{~d}, J=15.2 \mathrm{~Hz}$, $1 \mathrm{H}), 3.83(\mathrm{~s}, 3 \mathrm{H}), 3.30-3.28(\mathrm{~m}, 1 \mathrm{H}), 2.75(\mathrm{dd}, J=16.2,8.5 \mathrm{~Hz}, 1 \mathrm{H}), 2.63(\mathrm{dd}, J=$ 16.2, $6.0 \mathrm{~Hz}, 1 \mathrm{H}), 1.81$ (s, 3H), 1.42 (s, 3H). ${ }^{13} \mathrm{C} \mathrm{NMR}\left(150 \mathrm{MHz}, \mathrm{CDCl}_{3}\right) \delta$ 197.6, 172.1, 159.7, 151.4, 144.4, 136.5, 134.3, 131.3, 130.3, 129.5, 118.6, 113.9, 76.8, 70.7, 55.4, 46.2, 39.5, 24.8, 15.7. HRMS (ESI-TOF) m/z: $[\mathrm{M}+\mathrm{Na}]^{+} \mathrm{Calcd}$ for $\mathrm{C}_{21} \mathrm{H}_{22} \mathrm{NaO}_{5}{ }^{+}$ 377.1359, Found: 377.1345 .

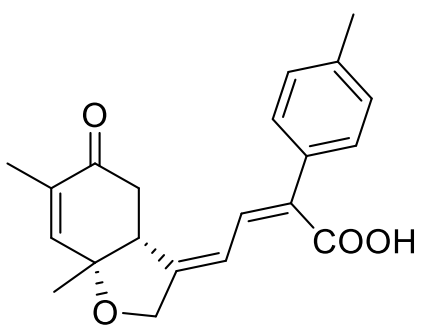

(Z)-4-((E)-6,7a-dimethyl-5-oxo-3a,4,5,7a-tetrahydrobenzofuran-3(2H)-ylidene)-2(p-tolyl)but-2-enoic acid (3jb).

White solid (46 mg, 68\%, m.p. $80-81{ }^{\circ} \mathrm{C}$, eluent: PE/EA = 2:1). ${ }^{1} \mathrm{H}$ NMR $(600 \mathrm{MHz}$, $\left.\mathrm{CDCl}_{3}\right) \delta 7.28(\mathrm{~d}, J=8.0 \mathrm{~Hz}, 2 \mathrm{H}), 7.18(\mathrm{~d}, J=7.9 \mathrm{~Hz}, 2 \mathrm{H}), 7.00-6.91(\mathrm{~m}, 1 \mathrm{H}), 6.81$ $(\mathrm{d}, J=12.0 \mathrm{~Hz}, 1 \mathrm{H}), 6.41(\mathrm{~m}, 1 \mathrm{H}), 4.59(\mathrm{~d}, J=15.1 \mathrm{~Hz}, 1 \mathrm{H}), 4.52(\mathrm{~d}, J=15.2 \mathrm{~Hz}, 1 \mathrm{H})$, $3.3-3.27(\mathrm{~m}, 1 \mathrm{H}), 2.74(\mathrm{dd}, J=16.2,8.5 \mathrm{~Hz}, 1 \mathrm{H}), 2.62(\mathrm{dd}, J=16.2,6.1 \mathrm{~Hz}, 1 \mathrm{H}), 2.37$ $(\mathrm{s}, 3 \mathrm{H}), 1.81(\mathrm{~d}, J=1.1 \mathrm{~Hz}, 3 \mathrm{H}), 1.42(\mathrm{~s}, 3 \mathrm{H}) .{ }^{13} \mathrm{C} \mathrm{NMR}\left(150 \mathrm{MHz}, \mathrm{CDCl}_{3}\right) \delta 197.7$, 171.9, 151.9, 144.5, 138.4, 136.6, 135.1, 135.0, 131.8, 129.3, 128.2, 118.7, 80.0, 70.8, 46.4, 39.7, 25.0, 21.4, 15.8.HRMS (ESI-TOF) m/z: $[\mathrm{M}+\mathrm{Na}]^{+}$Calcd for $\mathrm{C}_{21} \mathrm{H}_{22} \mathrm{NaO}_{4}{ }^{+}$ 361.1410, Found: 361.1393.<smiles>CC1=C[C@]2(C)OC/C(=C/C=C(\C(=O)O)c3ccc(F)cc3)[C@H]2CC1=O</smiles>

(Z)-4-((E)-6,7a-dimethyl-5-oxo-3a,4,5,7a-tetrahydrobenzofuran-3(2H)-ylidene)-2(4-fluorophenyl)but-2-enoic acid (3kb).

Yellow solid (54 mg, 79\%, m.p. $178-180{ }^{\circ} \mathrm{C}$, eluent: PE/EA = 2:1). ${ }^{1} \mathrm{H}$ NMR (400 $\left.\mathrm{MHz} \mathrm{CDCl}_{3}\right) \delta 7.35(\mathrm{dd}, J=8.7,5.3 \mathrm{~Hz}, 2 \mathrm{H}), 7.02(\mathrm{dt}, J=12.1,5.1 \mathrm{~Hz}, 3 \mathrm{H}), 6.81(\mathrm{~d}$, $J=12.0 \mathrm{~Hz}, 1 \mathrm{H}), 6.41(\mathrm{~d}, J=1.2 \mathrm{~Hz}, 1 \mathrm{H}), 4.59(\mathrm{~d}, J=15.2 \mathrm{~Hz}, 1 \mathrm{H}), 4.52(\mathrm{~d}, J=15.3$ 
$\mathrm{Hz}, 1 \mathrm{H}), 3.31-3.27(\mathrm{~m}, 1 \mathrm{H}), 2.75(\mathrm{dd}, J=16.2,8.3 \mathrm{~Hz}, 1 \mathrm{H}), 2.63(\mathrm{dd}, J=16.2,6.1$ $\mathrm{Hz}, 1 \mathrm{H}), 1.80(\mathrm{~m}, J=1.0 \mathrm{~Hz}, 3 \mathrm{H}), 1.42$ (s, 3H). ${ }^{13} \mathrm{C} \mathrm{NMR}\left(100 \mathrm{MHz}, \mathrm{CDCl}_{3}\right) \delta 197.7$, 172.0, $162.7(\mathrm{~d}, J=246.9 \mathrm{~Hz}), 152.8,144.6,136.6,136.4,134.2(\mathrm{~d}, J=3.5 \mathrm{~Hz}), 130.6$, $130.3(\mathrm{~d}, J=8.1 \mathrm{~Hz}), 118.6,115.4(\mathrm{~d}, J=21.6 \mathrm{~Hz}), 80.1,70.8,46.4,39.7,24.8,15.8$. ${ }^{19} \mathrm{~F} \mathrm{NMR}\left(565 \mathrm{MHz}, \mathrm{CDCl}_{3}\right) \delta-113.58--113.59$ (m, 1F). HRMS (ESI-TOF) m/z: [M $+\mathrm{Na}]^{+}$Calcd for $\mathrm{C}_{20} \mathrm{H}_{19} \mathrm{FNaO}_{4}{ }^{+}$365.1160, Found: 365.1150 .<smiles>CC1=C[C@]2(C)OC/C(=C/C=C(\C(=O)O)c3ccc(Br)cc3)[C@H]2CC1=O</smiles>

(Z)-2-(4-bromophenyl)-4-((E)-6,7a-dimethyl-5-oxo-3a,4,5,7atetrahydrobenzofuran-3(2H)-ylidene)but-2-enoic acid (3lb).

Yellow solid (66 mg, 82\%, m.p. $190-192{ }^{\circ} \mathrm{C}$, eluent: PE/EA = 2:1). ${ }^{1} \mathrm{H}$ NMR (400 $\left.\mathrm{MHz}, \mathrm{CDCl}_{3}\right) \delta 7.48(\mathrm{~d}, J=8.5 \mathrm{~Hz}, 2 \mathrm{H}), 7.25(\mathrm{~d}, J=8.5 \mathrm{~Hz}, 2 \mathrm{H}), 7.02-6.99(\mathrm{~m}, 1 \mathrm{H})$, $6.84(\mathrm{~d}, J=12.0 \mathrm{~Hz}, 1 \mathrm{H}), 6.41-6.40(\mathrm{~m}, 1 \mathrm{H}), 4.58(\mathrm{~d}, J=15.3 \mathrm{~Hz}, 1 \mathrm{H}), 4.52(\mathrm{~d}, J=$ $15.4 \mathrm{~Hz}, 1 \mathrm{H}), 3.30-3.27(\mathrm{~m}, 1 \mathrm{H}), 2.74(\mathrm{dd}, J=16.2,8.2 \mathrm{~Hz}, 1 \mathrm{H}), 2.62(\mathrm{dd}, J=16.2$, $6.1 \mathrm{~Hz}, 1 \mathrm{H}), 1.80(\mathrm{~m}, J=1.0 \mathrm{~Hz}, 3 \mathrm{H}), 1.43(\mathrm{~s}, 3 \mathrm{H}) .{ }^{13} \mathrm{C} \mathrm{NMR}\left(100 \mathrm{MHz}, \mathrm{CDCl}_{3}\right) \delta$ 197.6, 171.7, 153.4, 144.6, 137.1, 136.7, 136.6, 131.6, 130.5, 130.1, 122.5, 118.6, 80.2, 70.8, 46.4, 39.7, 24.8, 15.8. HRMS (ESI-TOF) m/z: $[\mathrm{M}+\mathrm{Na}]^{+}$Calcd for $\mathrm{C}_{20} \mathrm{H}_{19} \mathrm{BrNaO}_{4}{ }^{+} 425.0359$, Found: 425.0344.<smiles>CC1=C[C@]2(C)OC/C(=C/C=C(\C(=O)O)c3ccccc3[N+](=O)[O-])[C@H]2CC1=O</smiles>

(Z)-4-((E)-6,7a-dimethyl-5-oxo-3a,4,5,7a-tetrahydrobenzofuran-3(2H)-ylidene)-2(2-nitrophenyl)but-2-enoic acid (3mb).

Yellow solid (65 mg, 89\%, m.p. $112-113{ }^{\circ} \mathrm{C}$, eluent: PE/EA = 2:1). ${ }^{1} \mathrm{H}$ NMR (600 $\left.\mathrm{MHz}, \mathrm{CDCl}_{3}\right) \delta 8.14(\mathrm{~d}, J=8.2 \mathrm{~Hz}, 1 \mathrm{H}), 7.69-7.66(\mathrm{~m}, 1 \mathrm{H}), 7.56-7.49(\mathrm{~m}, 1 \mathrm{H}), 7.41$ $-7.39(\mathrm{~m}, 1 \mathrm{H}), 7.37-7.34(\mathrm{~m}, 1 \mathrm{H}), 6.82(\mathrm{~d}, J=12.0 \mathrm{~Hz}, 1 \mathrm{H}), 6.42(\mathrm{~m},, 1 \mathrm{H}), 4.64(\mathrm{~d}$, 
$J=15.6 \mathrm{~Hz}, 1 \mathrm{H}), 4.57(\mathrm{~d}, J=15.7 \mathrm{~Hz}, 1 \mathrm{H}), 3.29-3.22(\mathrm{~m}, 1 \mathrm{H}), 2.73(\mathrm{dd}, J=16.1,9.1$ $\mathrm{Hz}, 1 \mathrm{H}), 2.58(\mathrm{dd}, J=16.1,5.9 \mathrm{~Hz}, 1 \mathrm{H}), 1.80(\mathrm{~m}, 3 \mathrm{H}), 1.40(\mathrm{~s}, 3 \mathrm{H}) .{ }^{13} \mathrm{C}$ NMR $(150$ $\left.\mathrm{MHz}, \mathrm{CDCl}_{3}\right) \delta 197.7,169.6,154.8,147.9,144.4,138.5,136.6,134.8,134.1,132.7$, 129.3, 128.6, 124.9, 118.5, 80.1, 70.9, 46.4, 39.7, 24.9, 15.8. HRMS (ESI-TOF) m/z: $[\mathrm{M}+\mathrm{Na}]^{+}$Calcd for $\mathrm{C}_{20} \mathrm{H}_{19} \mathrm{NNaO}_{6}{ }^{+}$392.1105, Found: 392.1103 .<smiles>CC1=C[C@]2(C)OC/C(=C/C=C(\C(=O)O)c3cccc4ccccc34)[C@H]2CC1=O</smiles>

(Z)-4-((E)-6,7a-dimethyl-5-oxo-3a,4,5,7a-tetrahydrobenzofuran-3(2H)-ylidene)-2(naphthalen-1-yl)but-2-enoic acid (3nb).

Yellow solid (63 mg, 84\%, m.p. $99-101{ }^{\circ} \mathrm{C}$, eluent: PE/EA = 2:1). ${ }^{1} \mathrm{H}$ NMR (600 MHz, $\left.\mathrm{CDCl}_{3}\right) \delta 7.87(\mathrm{t}, J=9.0 \mathrm{~Hz}, 2 \mathrm{H}), 7.81(\mathrm{~d}, J=7.8 \mathrm{~Hz}, 1 \mathrm{H}), 7.53-7.47(\mathrm{~m}, 3 \mathrm{H}), 7.39$ $(\mathrm{d}, J=6.9 \mathrm{~Hz}, 1 \mathrm{H}), 7.29-7.27(\mathrm{~m}, 1 \mathrm{H}), 6.88(\mathrm{~d}, J=12.0 \mathrm{~Hz}, 1 \mathrm{H}), 6.37(\mathrm{~s}, 1 \mathrm{H}), 4.59$ $(\mathrm{d}, J=15.4 \mathrm{~Hz}, 1 \mathrm{H}), 4.52(\mathrm{~d}, J=15.5 \mathrm{~Hz}, 1 \mathrm{H}), 3.20-3.18(\mathrm{~m}, 1 \mathrm{H}), 2.68(\mathrm{dd}, J=16.2$, $8.4 \mathrm{~Hz}, 1 \mathrm{H}), 2.51(\mathrm{dd}, J=16.2,6.0 \mathrm{~Hz}, 1 \mathrm{H}), 1.78(\mathrm{~s}, 3 \mathrm{H}), 1.38(\mathrm{~s}, 3 \mathrm{H}) .{ }^{13} \mathrm{C}$ NMR $(150$ $\left.\mathrm{MHz}, \mathrm{CDCl}_{3}\right) \delta 197.4,171.9,171.8,153.8,144.4,140.4,136.6,136.6,133.6,132.2$, $129.7,129.6,128.8,128.6,127.4,126.6,126.2$, 125.5, 125.4, 118.5, 80.0, 70.8, 46.3, 39.8, 24.8, 15.8. HRMS (ESI-TOF) $\mathrm{m} / \mathrm{z}:[\mathrm{M}+\mathrm{Na}]^{+}$Calcd for $\mathrm{C}_{24} \mathrm{H}_{22} \mathrm{NaO}_{4}{ }^{+} 397.1410$, Found: 397.1397.<smiles>CC1=C[C@]2(C)OC/C(=C/C3=C(C(=O)O)CCC3)[C@H]2CC1=O</smiles>

(E)-2-((6,7a-dimethyl-5-oxo-3a,4,5,7a-tetrahydrobenzofuran-3(2H)ylidene)methyl)cyclopent-1-ene-1-carboxylic acid (3ob).

Yellow oil (34 mg, 59\%, eluent: PE/EA = 2:1). ${ }^{1} \mathrm{H} \mathrm{NMR}\left(600 \mathrm{MHz}, \mathrm{CDCl}_{3}\right) \delta 7.01$ (s, 1H), $6.42-6.43$ (m, 1H), 4.67 (d, $J=14.5 \mathrm{~Hz}, 1 \mathrm{H}), 4.52$ (d, $J=14.5 \mathrm{~Hz}, 1 \mathrm{H}), 3.30$ $3.24(\mathrm{~m}, 1 \mathrm{H}), 2.83-2.73(\mathrm{~m}, 2 \mathrm{H}), 2.70(\mathrm{~m}, 2 \mathrm{H}), 2.59$ (dd, $J=16.1,9.9 \mathrm{~Hz}, 1 \mathrm{H}), 2.50$ $(\mathrm{dd}, J=16.1,5.8 \mathrm{~Hz}, 1 \mathrm{H}), 1.94-1.89(\mathrm{~m}, 2 \mathrm{H}), 1.80$ (m, $J=1.2 \mathrm{~Hz}, 3 \mathrm{H}), 1.37$ (s, 3H). 
${ }^{13} \mathrm{C}$ NMR $\left(150 \mathrm{MHz}, \mathrm{CDCl}_{3}\right) \delta 198.1,170.5,152.8,148.0,144.2,136.5,130.4,117.3$, 80.0, 71.7, 47.1, 39.4, 37.2, 33.3, 25.4, 22.0, 15.8. HRMS (ESI-TOF) m/z: $[\mathrm{M}+\mathrm{Na}]^{+}$ Calcd for $\mathrm{C}_{17} \mathrm{H}_{20} \mathrm{NaO}_{4}{ }^{+}$311.1254, Found: 311.1248.

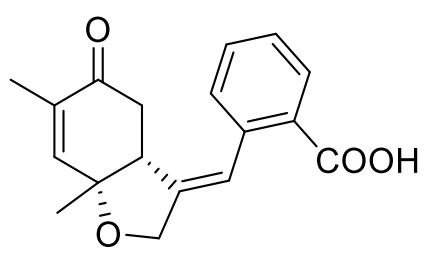

(E)-2-((6,7a-dimethyl-5-oxo-3a,4,5,7a-tetrahydrobenzofuran-3(2H)ylidene)methyl)benzoic acid (3pb).

Yellow oil (38 mg, 63\%, eluent: PE/EA = 2:1). ${ }^{1} \mathrm{H} \mathrm{NMR}\left(600 \mathrm{MHz}, \mathrm{CDCl}_{3}\right) \delta 8.11(\mathrm{~d}$, $J=7.8 \mathrm{~Hz}, 1 \mathrm{H}), 7.53-7.51(\mathrm{~m}, 1 \mathrm{H}), 7.39(\mathrm{t}, J=7.6 \mathrm{~Hz}, 1 \mathrm{H}), 7.29(\mathrm{~d}, J=7.6 \mathrm{~Hz}, 1 \mathrm{H})$, $6.84(\mathrm{~d}, J=1.7 \mathrm{~Hz}, 1 \mathrm{H}), 6.30(\mathrm{~s}, 1 \mathrm{H}), 4.57(\mathrm{~d}, J=13.3 \mathrm{~Hz}, 1 \mathrm{H}), 4.48-4.46(\mathrm{~m}, 1 \mathrm{H})$, $3.17(\mathrm{~m}, 1 \mathrm{H}), 2.27(\mathrm{dd}, J=16.6,5.5 \mathrm{~Hz}, 1 \mathrm{H}), 2.22(\mathrm{dd}, J=16.5,5.1 \mathrm{~Hz}, 1 \mathrm{H}), 1.72(\mathrm{~m}$, 3H), $1.46(\mathrm{~s}, 3 \mathrm{H}) .{ }^{13} \mathrm{C} \mathrm{NMR}\left(150 \mathrm{MHz}, \mathrm{CDCl}_{3}\right) \delta 197.4,171.2,145.5,141.7,139.2$, 136.4, 132.9, 131.8, 129.9, 128.0, 127.7, 122.5, 81.0, 71.0, 46.6, 37.2, 23.9, 15.7. HRMS (ESI-TOF) m/z: [M + H] $]^{+}$Calcd for $\mathrm{C}_{18} \mathrm{H}_{18} \mathrm{NaO}_{4}{ }^{+}$321.1097, Found: 321.2005.<smiles>CC1=C[C@]2(C)OC/C(=C\c3ccc4ccccc4c3C(=O)O)[C@H]2CC1=O</smiles>

(E)-2-((6,7a-dimethyl-5-oxo-3a,4,5,7a-tetrahydrobenzofuran-3(2H)ylidene)methyl)-1-naphthoic acid (3qb).

Yellow oil (55 mg, 78\%, eluent: PE/EA = 2:1). ${ }^{1} \mathrm{H} \mathrm{NMR}\left(600 \mathrm{MHz}, \mathrm{CDCl}_{3}\right) \delta 8.05(\mathrm{~d}$, $J=8.4 \mathrm{~Hz}, 1 \mathrm{H}), 7.90(\mathrm{~d}, J=8.4 \mathrm{~Hz}, 1 \mathrm{H}), 7.86(\mathrm{~d}, J=8.0 \mathrm{~Hz}, 1 \mathrm{H}), 7.57(\mathrm{t}, J=7.5 \mathrm{~Hz}$, 1H), $7.52(\mathrm{t}, J=7.4 \mathrm{~Hz}, 1 \mathrm{H}), 7.36(\mathrm{~d}, J=8.4 \mathrm{~Hz}, 1 \mathrm{H}), 6.73(\mathrm{~s}, 1 \mathrm{H}), 6.33$ (s, 1H), 4.60 $(\mathrm{d}, J=13.6 \mathrm{~Hz}, 1 \mathrm{H}), 4.44(\mathrm{~d}, J=13.5 \mathrm{~Hz}, 1 \mathrm{H}), 3.32(\mathrm{~m}, 1 \mathrm{H}), 2.43-2.28(\mathrm{~m}, 2 \mathrm{H}), 1.74$ (s, 3H), 1.50 (s, 3H). ${ }^{13} \mathrm{C}$ NMR $\left(150 \mathrm{MHz}, \mathrm{CDCl}_{3}\right) \delta 199.0,171.9,145.9,145.5,136.6$, $132.5,132.2$, 130.5, 129.9, 129.8, 128.3, 127.9, 126.7, 126.0, 125.3, 120.3, 81.4, 70.9, 47.1, 37.4, 23.8, 15.7. HRMS (ESI-TOF) m/z: $[\mathrm{M}+\mathrm{H}]^{+}$Calcd for $\mathrm{C}_{22} \mathrm{H}_{21} \mathrm{O}_{4}{ }^{+}$349.1434, Found: 349.1421. 
$\sum_{\bar{O}=1}^{\mathrm{COOH}}$

2-benzyl-4-((3R,3aR,7aR)-7a-methyl-5-oxooctahydrobenzofuran-3-yl)butanoic acid (4aa)

Yellow oil (24 mg, 72\%, eluent: PE/EA = 2:1), inseparable diastereoisomers (3:1 dr). 1H NMR (600 MHz, CDCl3) $\delta 7.32-7.27$ (m, 2H), $7.24-7.20(\mathrm{~m}, 1 \mathrm{H}), 7.19-7.14$ (m, 2H), $3.99-3.93(\mathrm{~m}, 1 \mathrm{H}), 3.46-3.28(\mathrm{~m}, 1 \mathrm{H}), 3.05-2.94(\mathrm{~m}, 1 \mathrm{H}), 2.77-2.61(\mathrm{~m}$, $2 \mathrm{H}), 2.53-2.43(\mathrm{~m}, 1 \mathrm{H}), 2.42-1.76(\mathrm{~m}, 6 \mathrm{H}), 1.70-1.40(\mathrm{~m}, 3 \mathrm{H}), 1.33-1.26(\mathrm{~m}$, $3 \mathrm{H}) .{ }^{13} \mathrm{C}$ NMR $(150 \mathrm{MHz}, \mathrm{CDCl} 3) \delta 213.4,213.3,212.6,179.8,138.7,138.6,128.9$, $128.6,128.5,126.6,126.6,82.0,82.0,80.9,80.7,71.0,70.9,70.4,70.3,49.3,49.3,47.4$ 47.3, 47.3, 47.2, 47.2, 47.0, 45.2, 45.0, 42.3, 42.2, 41.4, 41.1, 38.2, 38.0, 37.8, 37.6, $35.7,34.9,34.3,32.9,30.2,30.1,30.1,29.8,29.8,27.4,27.4,27.6,24.9,24.8$. HRMS (ESI-TOF) m/z: [M + Na $]^{+}$Calcd for $\mathrm{C}_{20} \mathrm{H}_{26} \mathrm{NaO}_{4}{ }^{+}$353.1723, Found: 353.1722 .<smiles>CC(=O)/C=C(\CO)c1cc(O)c(C)cc1C</smiles>

methyl $(2 Z, 4 E)$-2-benzyl-6-hydroxy-5-(5-hydroxy-2,4-dimethylphenyl)hexa-2,4dienoate (5ab).

Yellow oil (70 mg, 99\%, eluent: PE/EA $=2: 1),{ }^{1} \mathrm{H}$ NMR $\left(600 \mathrm{MHz}, \mathrm{CDCl}_{3}\right) \delta 7.47(\mathrm{~d}$, $J=11.6 \mathrm{~Hz}, 1 \mathrm{H}), 7.19(\mathrm{t}, J=7.4 \mathrm{~Hz}, 2 \mathrm{H}), 7.14(\mathrm{t}, J=7.3 \mathrm{~Hz}, 1 \mathrm{H}), 7.06(\mathrm{~d}, J=7.3 \mathrm{~Hz}$, 2H), $6.95(\mathrm{~s}, 1 \mathrm{H}), 6.47(\mathrm{~s}, 1 \mathrm{H}), 6.27(\mathrm{~d}, J=11.6 \mathrm{~Hz}, 1 \mathrm{H}), 4.38(\mathrm{~s}, 2 \mathrm{H}), 3.77(\mathrm{~s}, 3 \mathrm{H})$, $3.53(\mathrm{~s}, 2 \mathrm{H}), 2.20(\mathrm{~s}, 3 \mathrm{H}), 2.05(\mathrm{~s}, 3 \mathrm{H}) .{ }^{13} \mathrm{C} \mathrm{NMR}\left(150 \mathrm{MHz}, \mathrm{CDCl}_{3}\right) \delta$ 155.8, 150.9, $139.9,139.4,134.4,132.7,129.1,128.7,128.4,127.4,126.5,126.3,122.6,110.6,67.0$, 55.6, 40.2, 18.6, 16.0. HRMS (ESI-TOF) m/z: $[\mathrm{M}+\mathrm{Na}]^{+}$Chemical Formula: $\mathrm{C}_{22} \mathrm{H}_{24} \mathrm{NaO}_{4}{ }^{+}$375.1567, Found: 375.1556. 
<smiles>COC(=O)/C(=C\C=C1/CO[C@]2(C)C=C(C)C(=O)C[C@H]12)Cc1ccccc1</smiles>

methyl(Z)-2-benzyl-4-((E)-6,7a-dimethyl-5-oxo-3a,4,5,7a-tetrahydrobenzofuran3(2H)-ylidene)but-2-enoate (6ab).

Yellow oil (350 mg, 99\%, eluent: PE/EA = 6:1), ${ }^{1} \mathrm{H}$ NMR $\left(600 \mathrm{MHz}, \mathrm{CDCl}_{3}\right) \delta 7.31(\mathrm{t}$, $J=7.6 \mathrm{~Hz}, 2 \mathrm{H}), 7.22(\mathrm{t}, J=7.4 \mathrm{~Hz}, 1 \mathrm{H}), 7.19(\mathrm{~d}, J=7.3 \mathrm{~Hz}, 2 \mathrm{H}), 6.97-7.00(\mathrm{~m}, 1 \mathrm{H})$, $6.42(\mathrm{~d}, J=11.8 \mathrm{~Hz}, 1 \mathrm{H}), 6.37(\mathrm{~d}, J=1.2 \mathrm{~Hz}, 1 \mathrm{H}), 4.51(\mathrm{~d}, J=14.8 \mathrm{~Hz}, 1 \mathrm{H}), 4.45(\mathrm{~d}$, $J=14.9 \mathrm{~Hz}, 1 \mathrm{H}), 3.70(\mathrm{~s}, 3 \mathrm{H}), 3.68(\mathrm{~s}, 2 \mathrm{H}), 3.13-3.10(\mathrm{~m}, 1 \mathrm{H}), 2.60(\mathrm{dd}, J=16.2,8.1$ $\mathrm{Hz}, 1 \mathrm{H}), 2.49$ (dd, $J=16.2,6.0 \mathrm{~Hz}, 1 \mathrm{H}), 1.79(\mathrm{~m}, J=1.2 \mathrm{~Hz}, 3 \mathrm{H}), 1.39(\mathrm{~s}, 3 \mathrm{H}) .{ }^{13} \mathrm{C}$ $\mathrm{NMR}\left(150 \mathrm{MHz}, \mathrm{CDCl}_{3}\right) \delta 197.6,167.6,150.2,144.6,139.1,136.5,135.3,130.5,129.0$, 128.7, 126.6, 118.4, 80.0, 70.7, 51.7, 46.1, 40.6, 39.6, 24.8, 15.8. HRMS (ESI-TOF) m/z: $[\mathrm{M}+\mathrm{Na}]^{+}$Chemical Formula: $\mathrm{C}_{22} \mathrm{H}_{24} \mathrm{NaO}_{4}{ }^{+} 375.1567$, Found: 375.1560 .<smiles>COC(=O)/C=C/C=C1C(=O)O[C@]2(C)C=C(C)C(=O)C[C@H]12</smiles>

(Z)-2-benzyl-4-((E)-6,7a-dimethyl-2,5-dioxo-3a,4,5,7a-tetrahydrobenzofuran3(2H)-ylidene)but-2-enoic acid (7ab).

Yellow oil (46 mg, 63\%, eluent: PE/EA = 2:1), ${ }^{1} \mathrm{H}$ NMR (600 MHz, $\left.\mathrm{CDCl}_{3}\right) \delta 7.91$ (dd, $J=12.2,1.9 \mathrm{~Hz}, 1 \mathrm{H}), 7.35(\mathrm{t}, J=7.6 \mathrm{~Hz}, 2 \mathrm{H}), 7.28-7.27(\mathrm{~m}, 1 \mathrm{H}), 7.20(\mathrm{~d}, J=7.2 \mathrm{~Hz}$, 2H), $6.48(\mathrm{~d}, J=1.3 \mathrm{~Hz}, 1 \mathrm{H}), 6.37(\mathrm{~d}, J=12.2 \mathrm{~Hz}, 1 \mathrm{H}), 3.80(\mathrm{~s}, 3 \mathrm{H}), 3.79(\mathrm{~s}, 2 \mathrm{H}), 3.39$ - $3.34(\mathrm{~m}, 1 \mathrm{H}), 2.57$ (dd, $J=16.0,6.3 \mathrm{~Hz}, 1 \mathrm{H}), 2.48$ (dd, $J=16.0,10.1 \mathrm{~Hz}, 1 \mathrm{H}), 1.85$ $(\mathrm{d}, J=1.2 \mathrm{~Hz}, 3 \mathrm{H}), 1.53$ (s, 3H). ${ }^{13} \mathrm{C} \mathrm{NMR}\left(150 \mathrm{MHz}, \mathrm{CDCl}_{3}\right) \delta 196.1,169.1,166.7$, 141.5, 140.5, 137.4, 137.3, 133.8, 133.0, 130.5, 129.2, 128.9, 127.1, 78.8, 52.4, 42.7, 41.0, 39.3, 27.8, 15.9. HRMS (ESI-TOF) m/z: $[\mathrm{M}+\mathrm{Na}]^{+}$Chemical Formula: $\mathrm{C}_{22} \mathrm{H}_{22} \mathrm{NaO}_{5}{ }^{+} 389.1359$, Found: 389.1350 . 


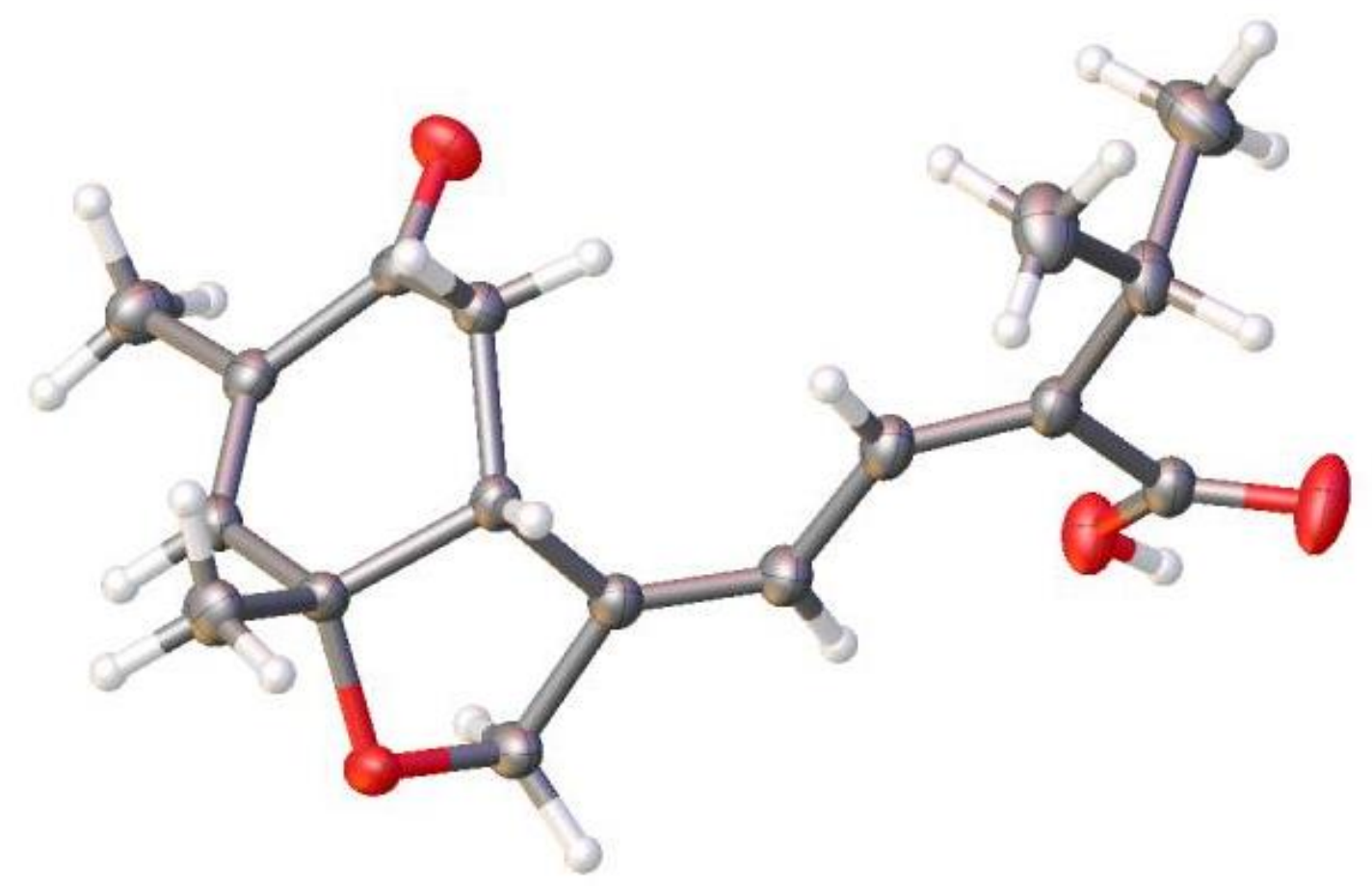

Table S1 Crystal data and structure refinement for 3eb

Identification code

Empirical formula

Formula weight

Temperature/K

Crystal system

Space group

$\mathrm{a} / \AA$

$\mathrm{b} / \AA$

c/

$\alpha^{\circ}$

$\beta /{ }^{\circ}$

$\gamma /{ }^{\circ}$

Volume $/ \AA^{3}$

Z 3eb

$\mathrm{C}_{17} \mathrm{H}_{22} \mathrm{O}_{4}$

290.34

170

monoclinic

$\mathrm{P} 2{ }_{1} / \mathrm{c}$

12.1876(2)

11.1367(2)

11.3580(2)

90

91.296(2)

90

1541.22(5)

4 
$\rho_{\text {calc }} \mathrm{g} / \mathrm{cm}^{3}$

$\mu / \mathrm{mm}^{-1}$

$\mathrm{F}(000)$

Crystal size $/ \mathrm{mm}^{3}$

Radiation

$2 \Theta$ range for data collection $/{ }^{\circ}$

Index ranges

Reflections collected

Independent reflections

Data/restraints/parameters

Goodness-of-fit on $\mathrm{F}^{2}$

Final $\mathrm{R}$ indexes $[\mathrm{I}>=2 \sigma(\mathrm{I})]$

Final R indexes [all data]

Largest diff. peak/hole / e $\AA^{-3}$
1.251

0.716

624.0

$0.2 \times 0.15 \times 0.15$

$\operatorname{CuK} \alpha(\lambda=1.54184)$

7.256 to 142.752

$-14 \leq \mathrm{h} \leq 14,-4 \leq \mathrm{k} \leq 13,-13 \leq 1 \leq 13$

6545

$2936\left[R_{\text {int }}=0.0212, R_{\text {sigma }}=0.0260\right]$

2936/0/198

1.029

$\mathrm{R}_{1}=0.0457, \mathrm{wR}_{2}=0.1213$

$\mathrm{R}_{1}=0.0501, \mathrm{wR}_{2}=0.1263$

$0.34 /-0.24$ 
NMR Spectra
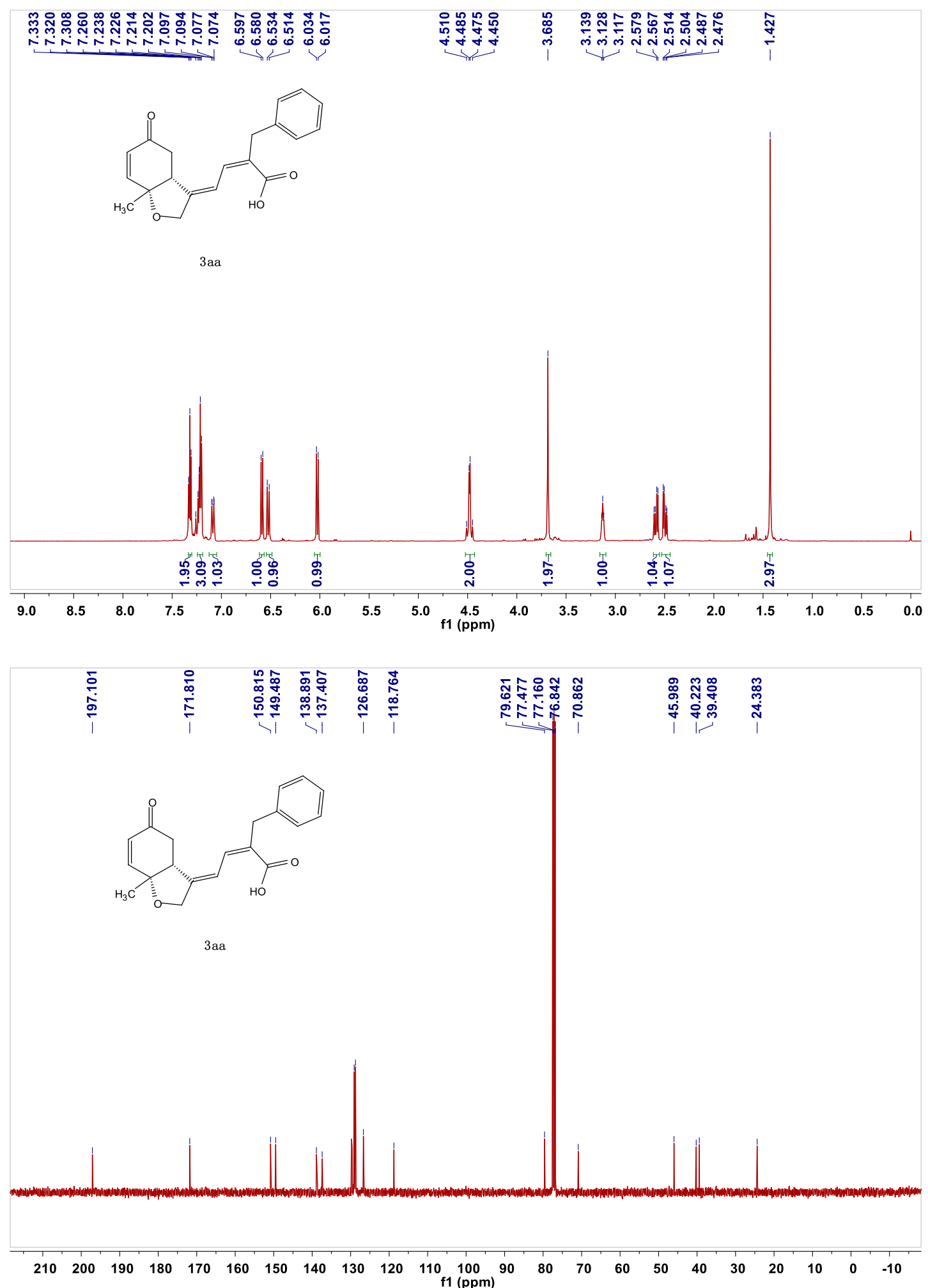

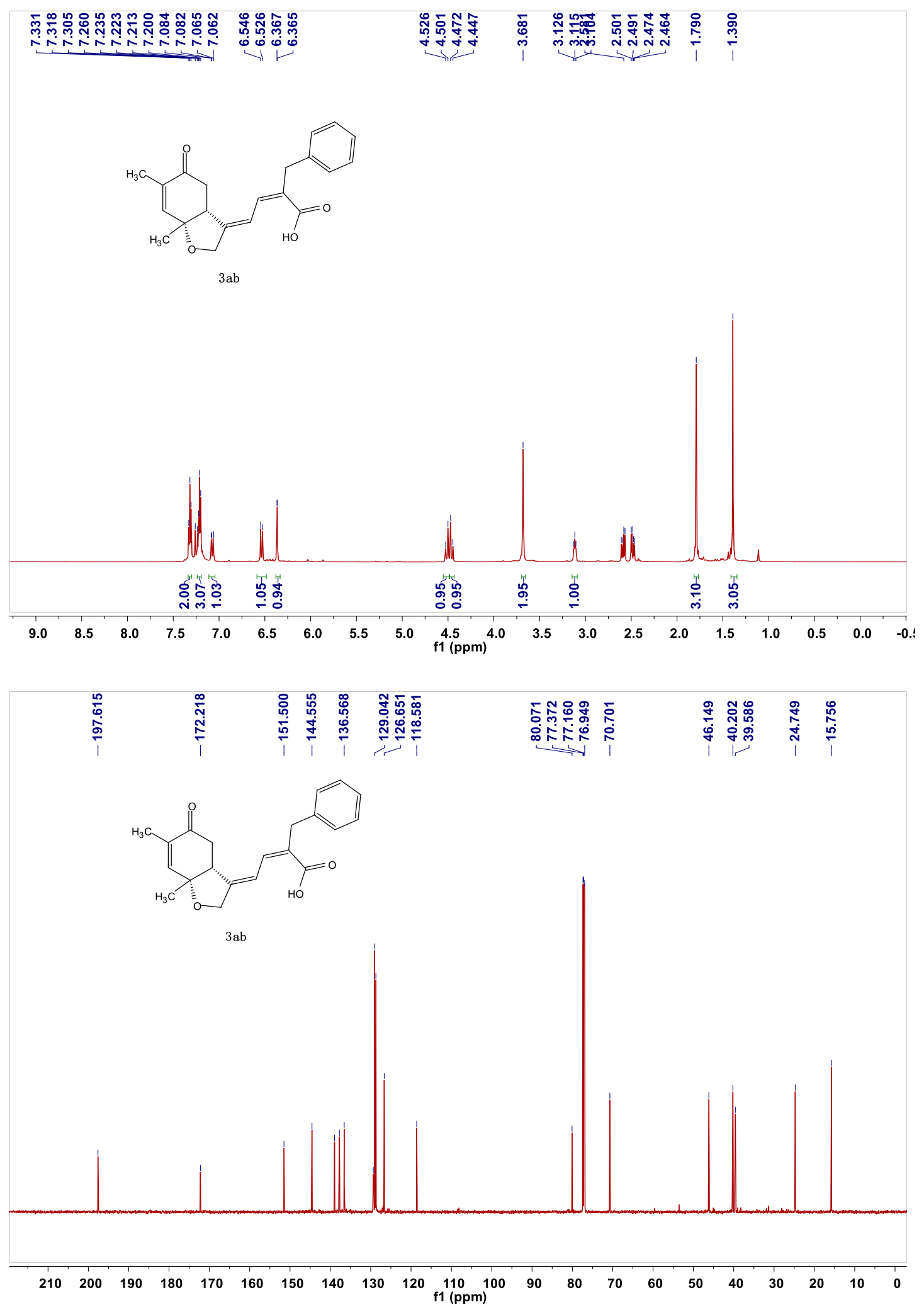

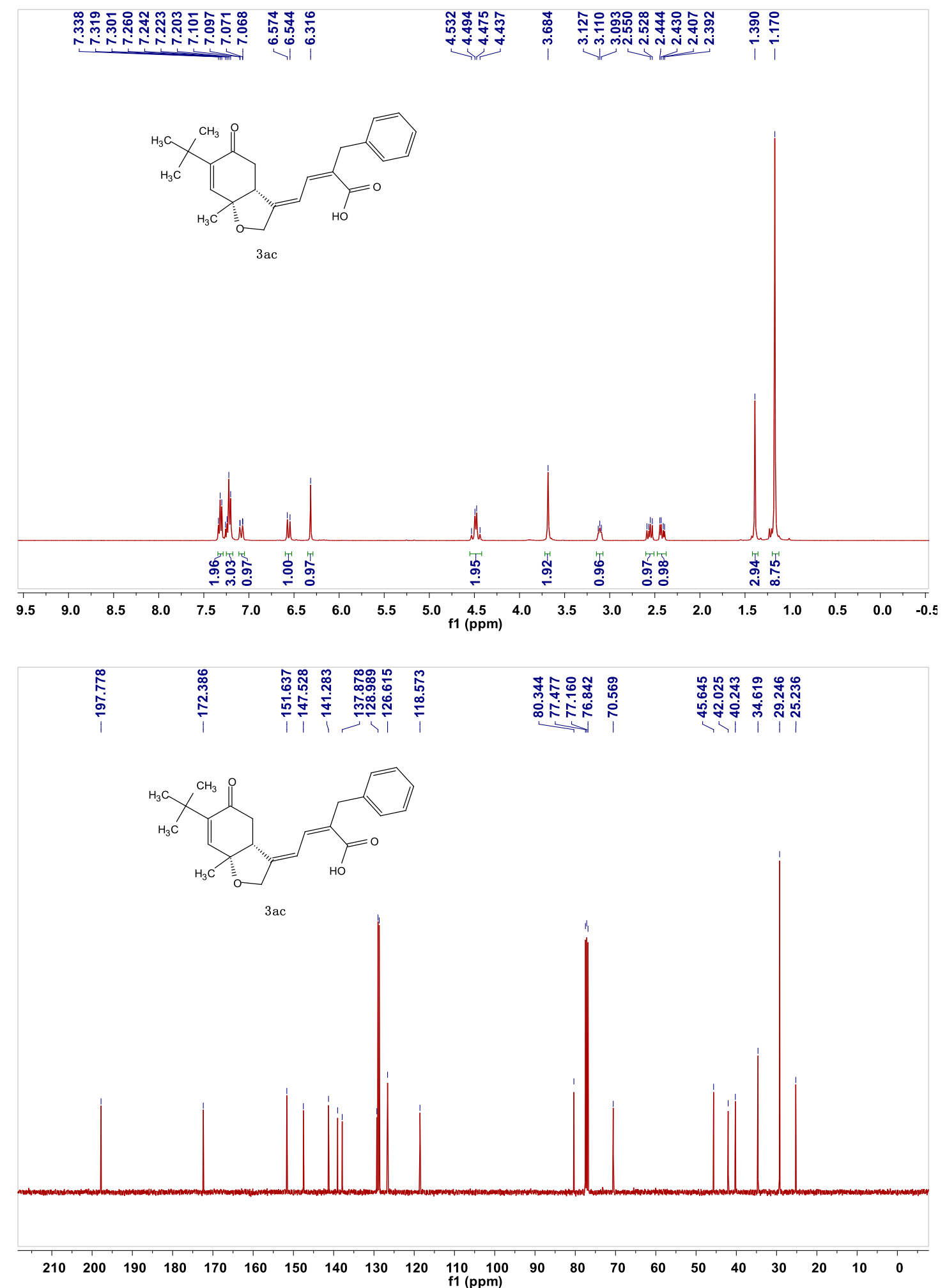


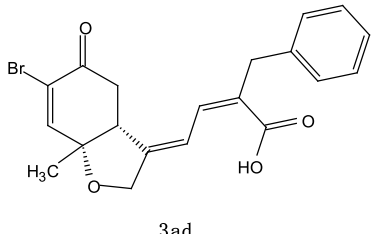

$3 \mathrm{ad}$

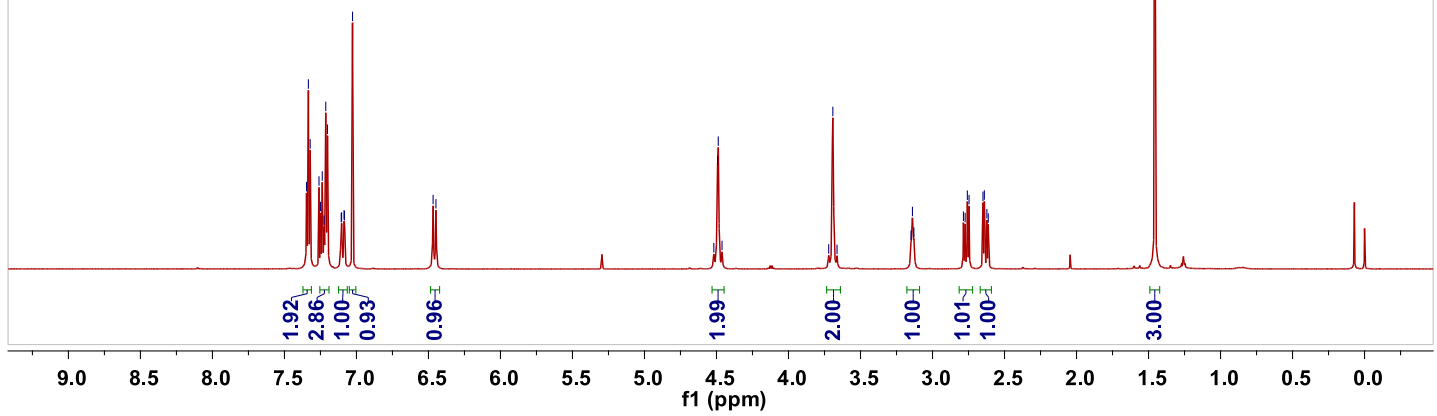

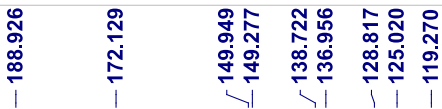

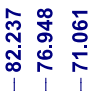

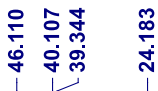

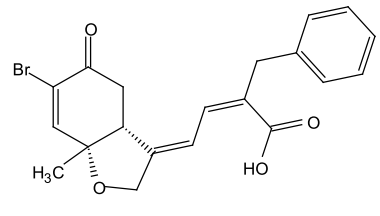

$3 a d$
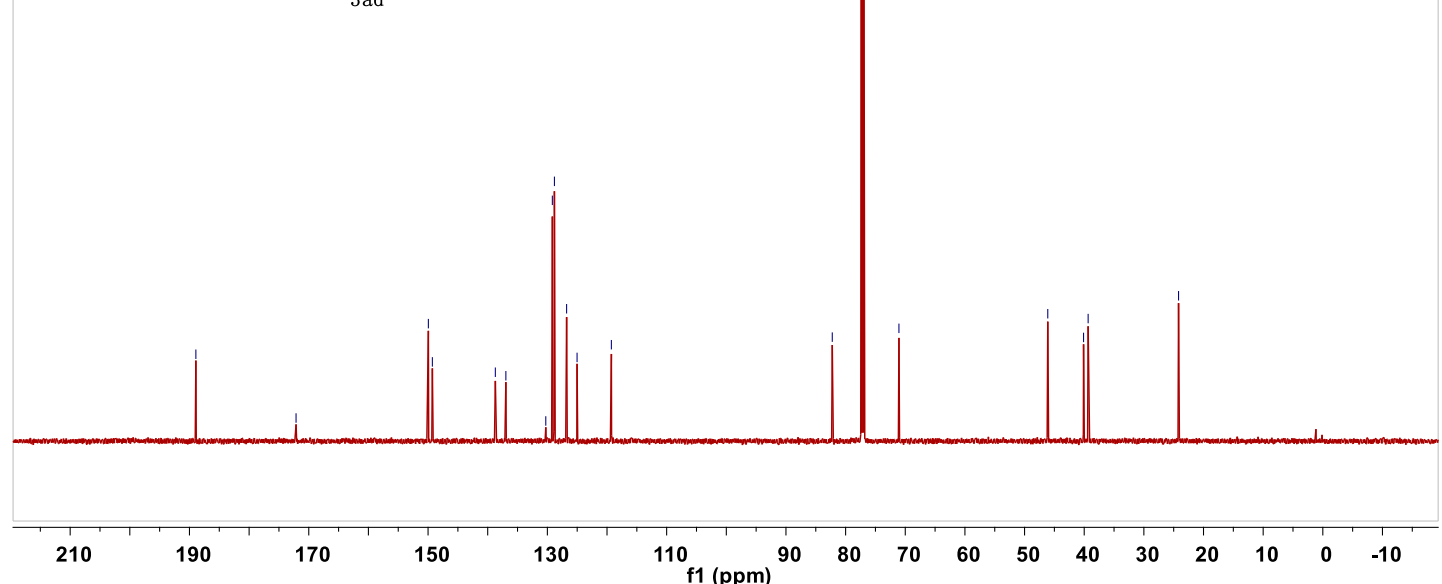

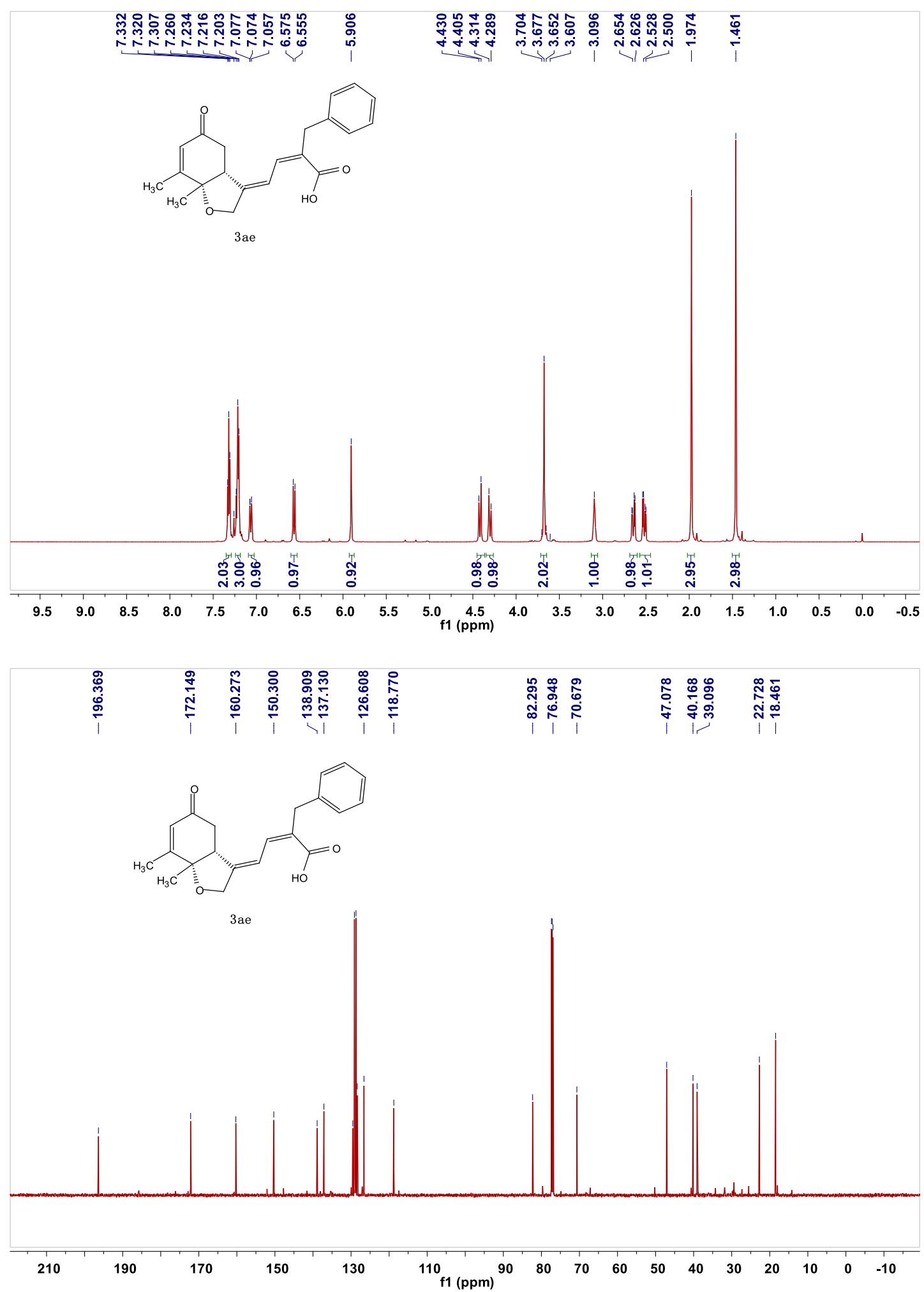

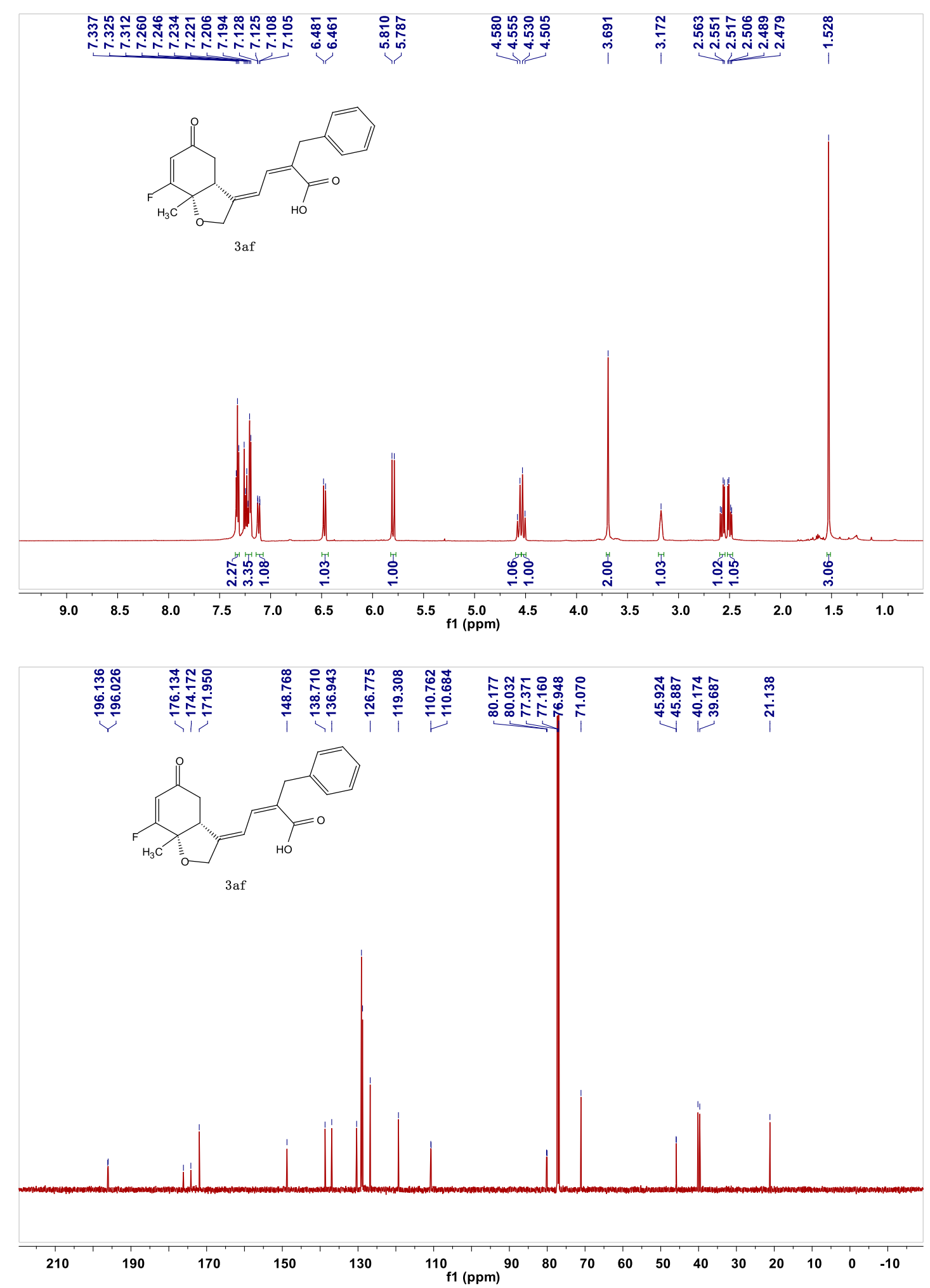

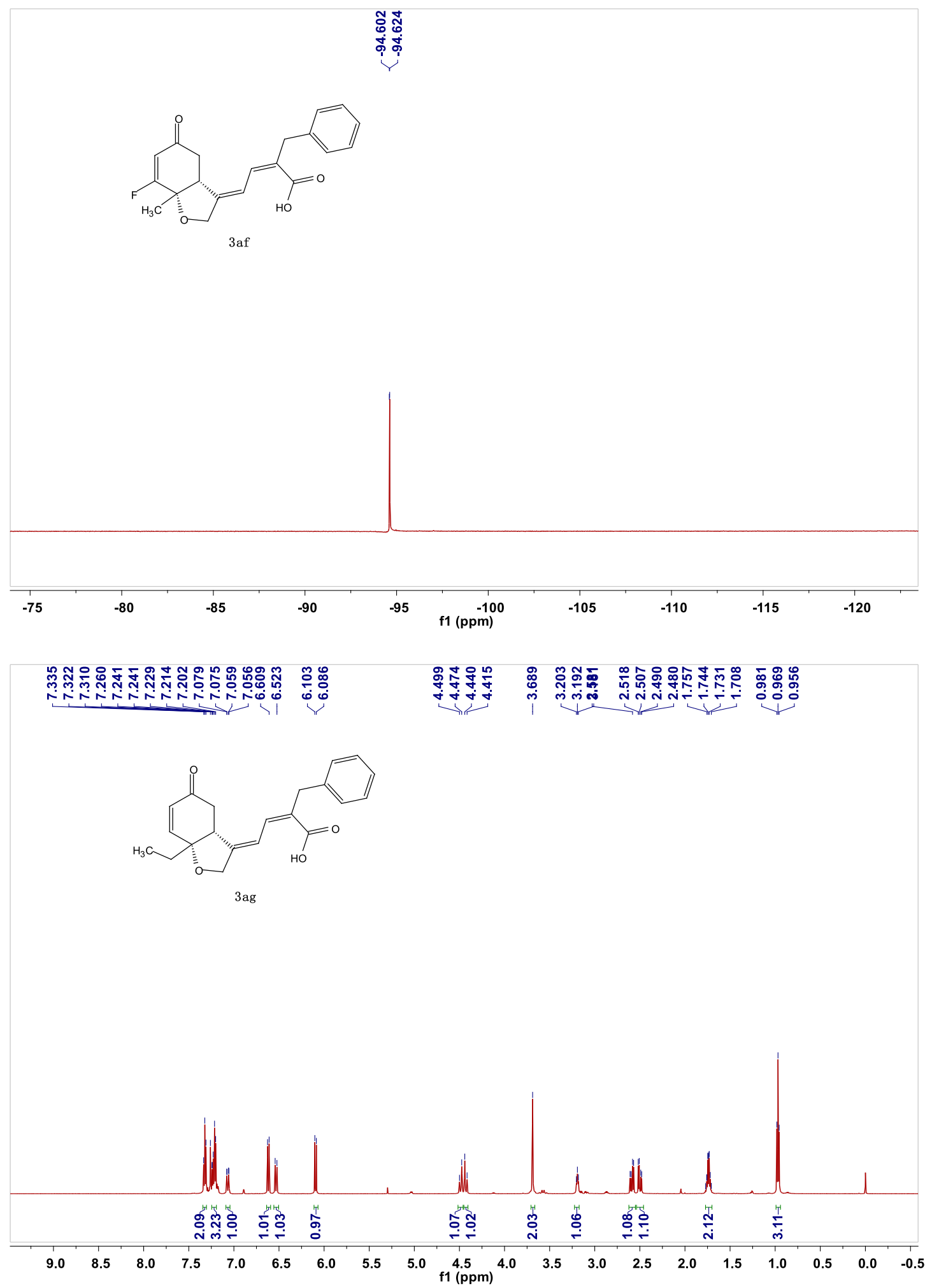

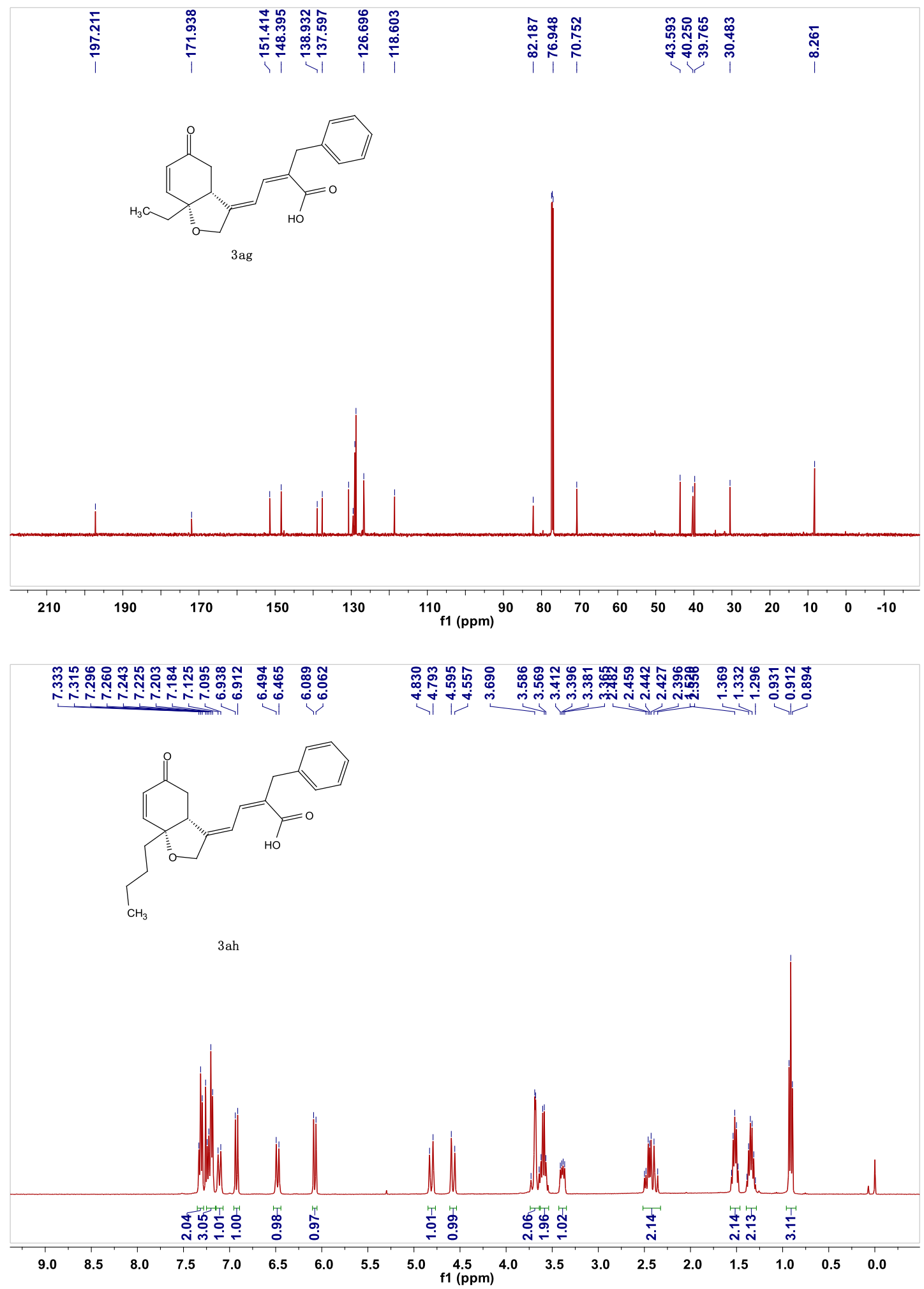


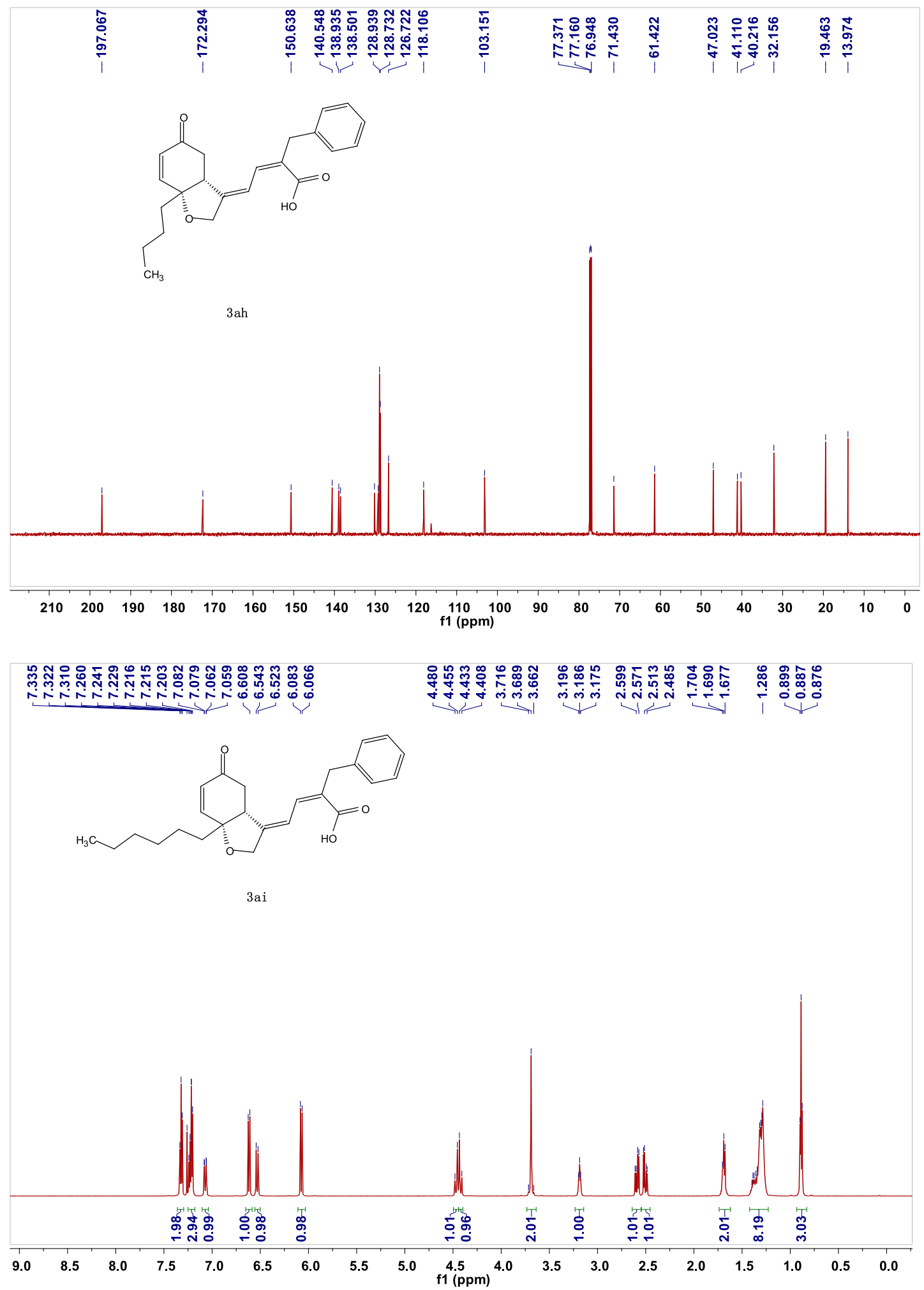



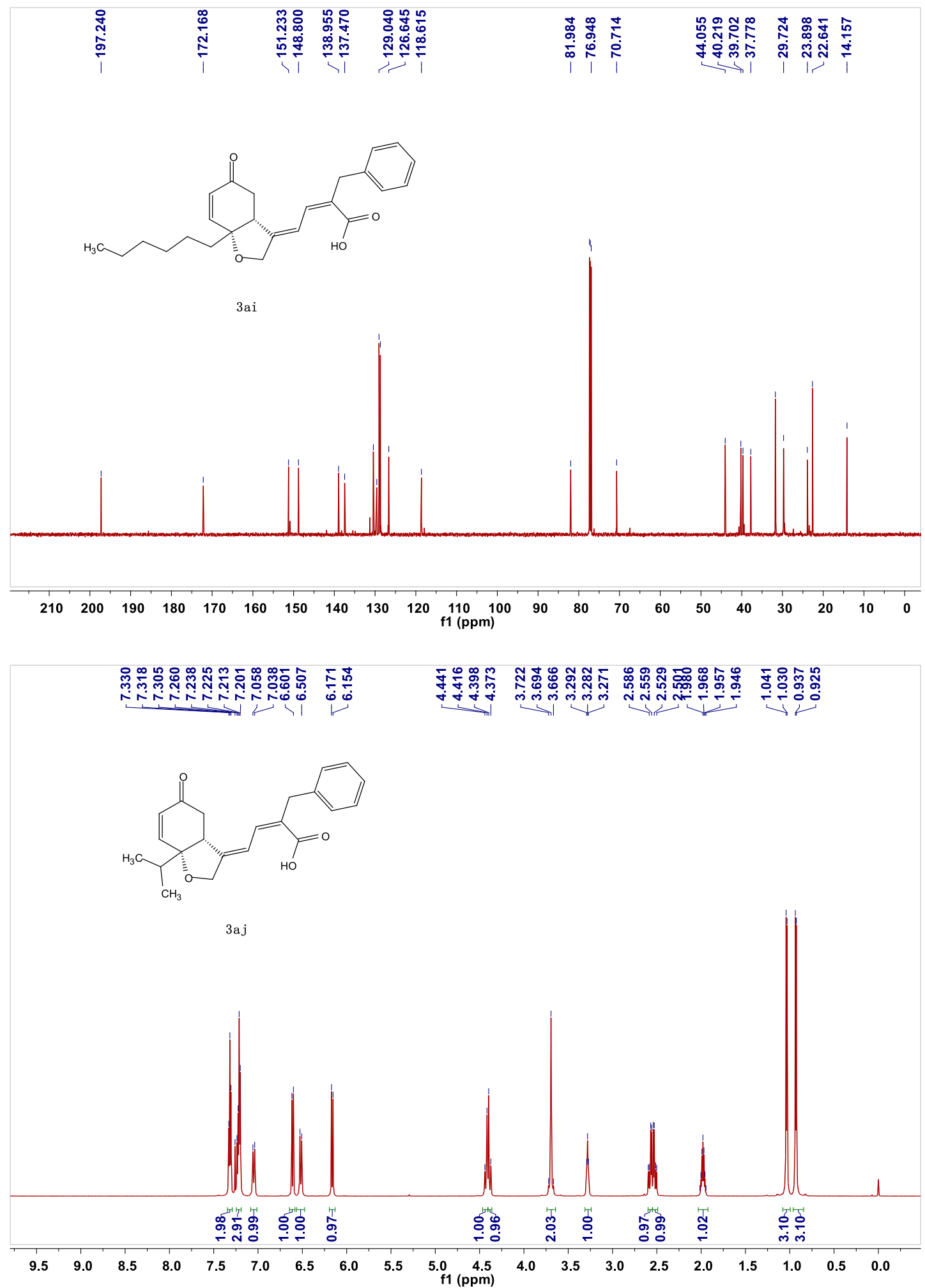

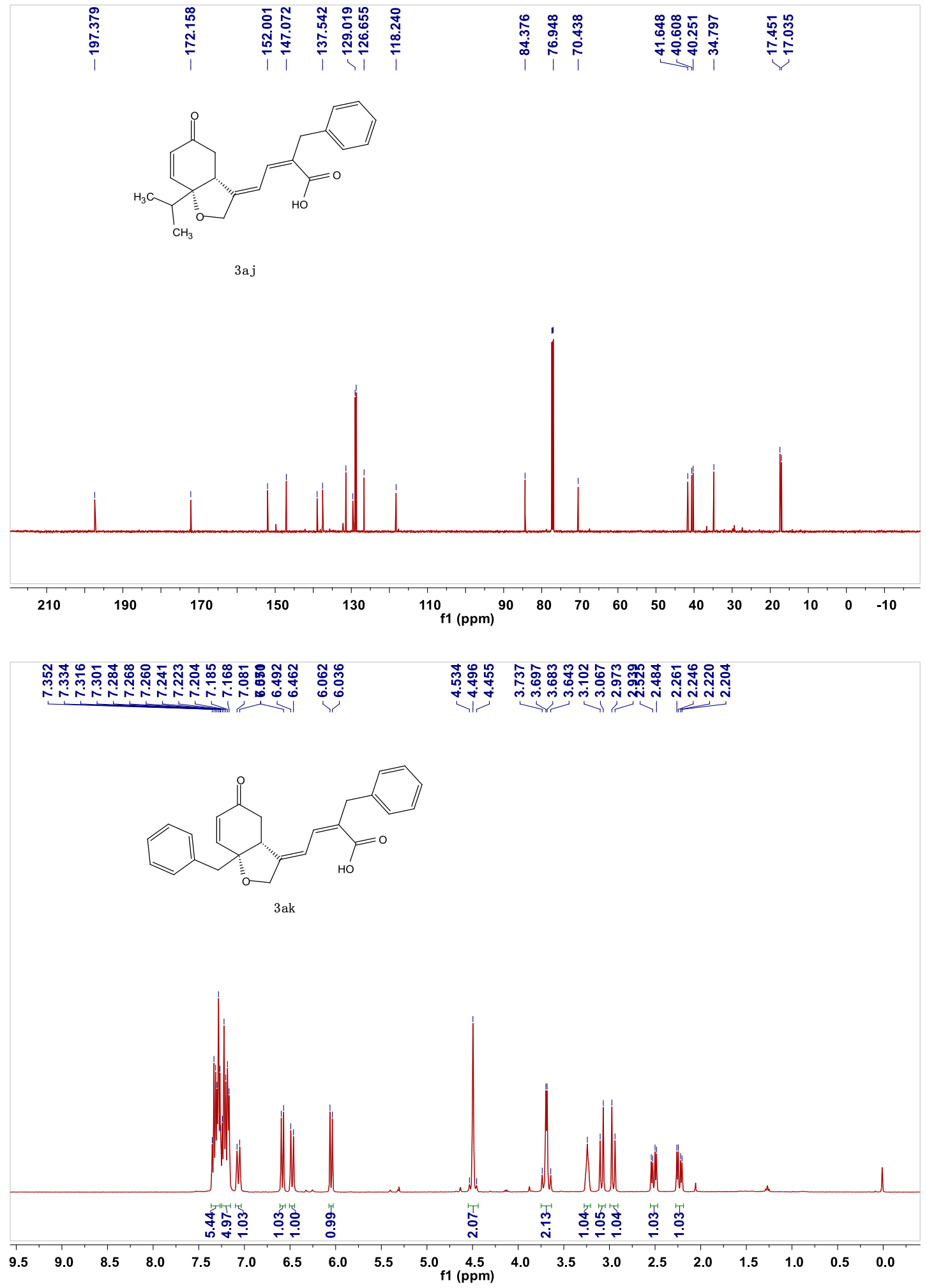

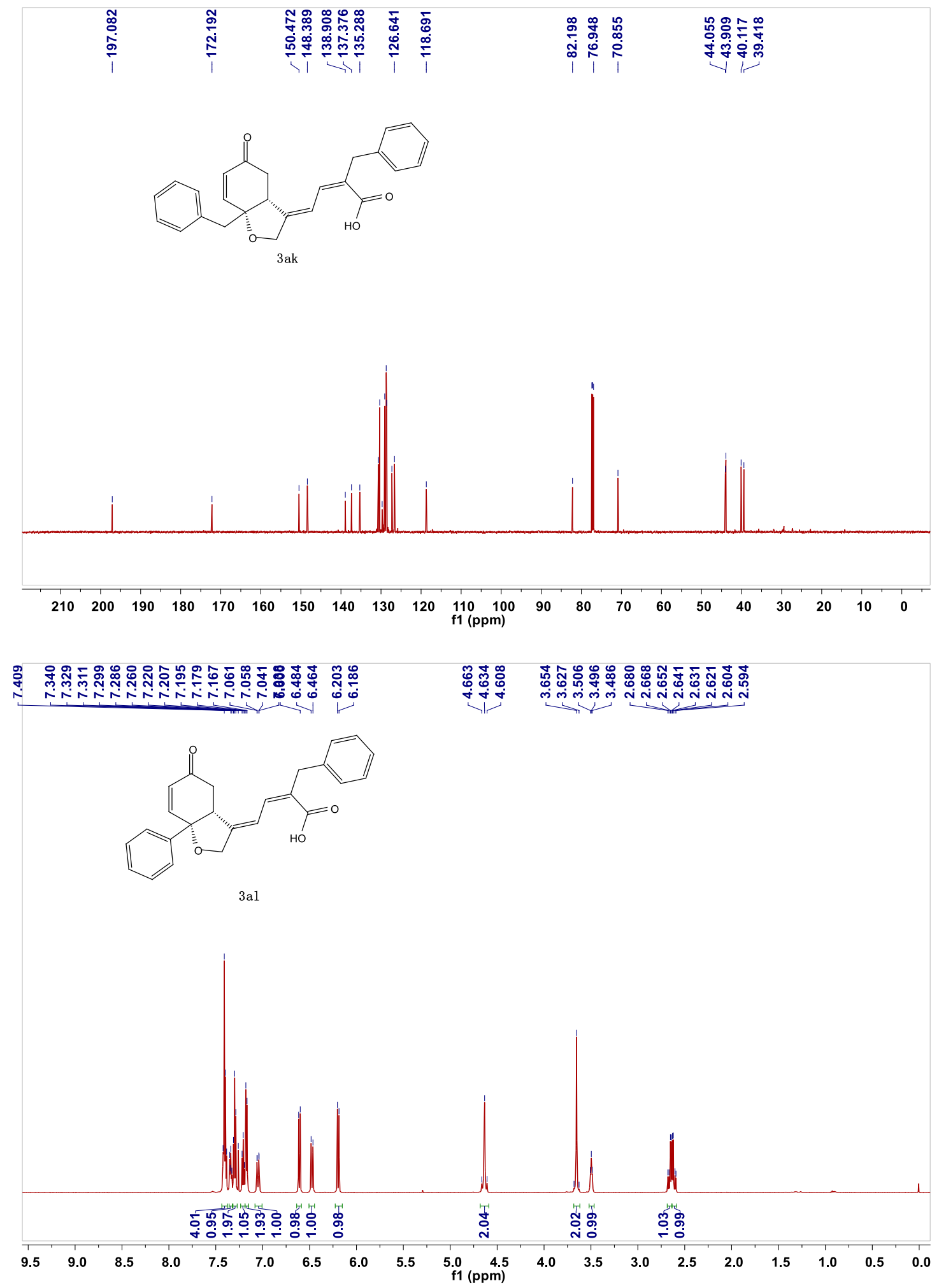

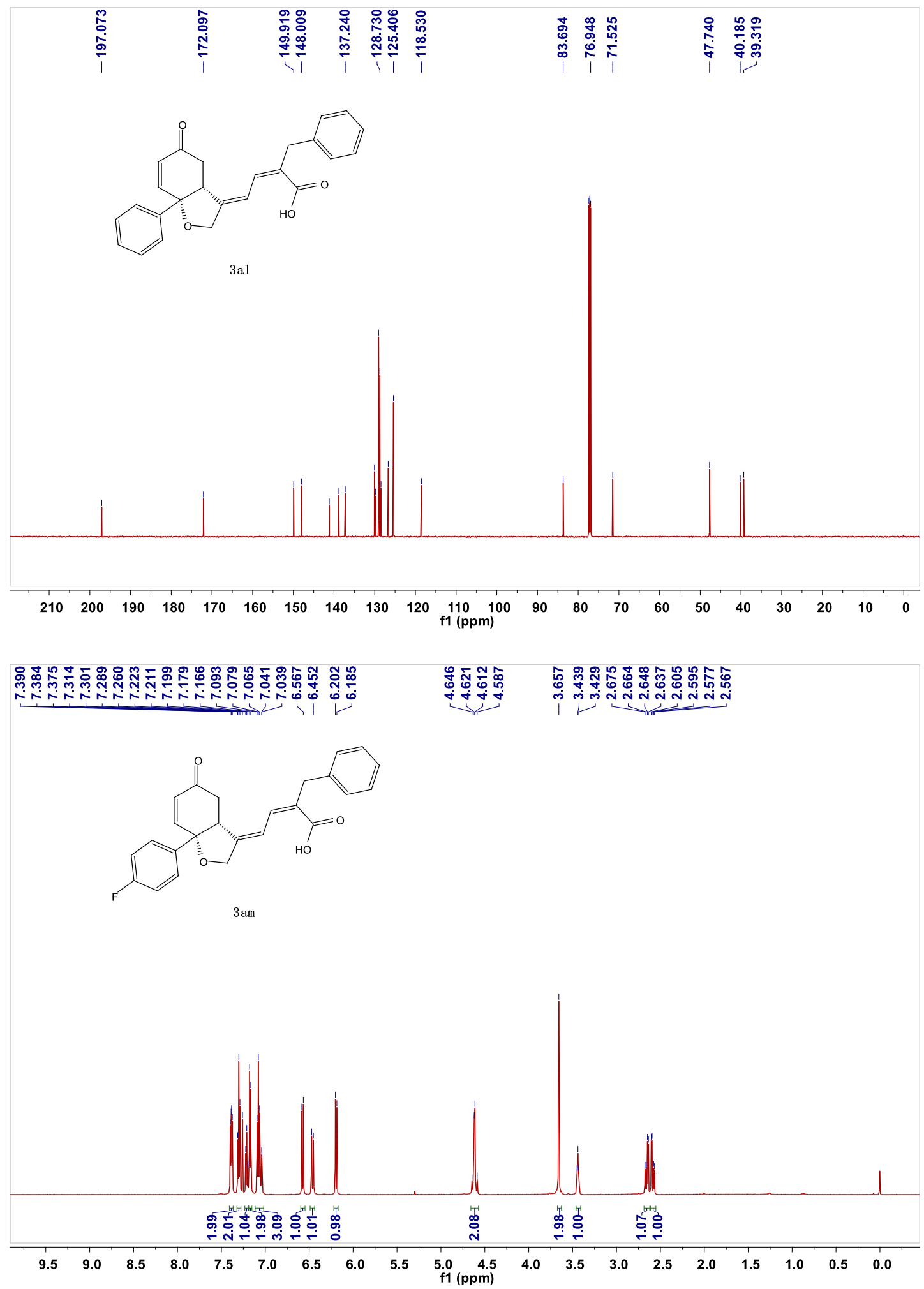

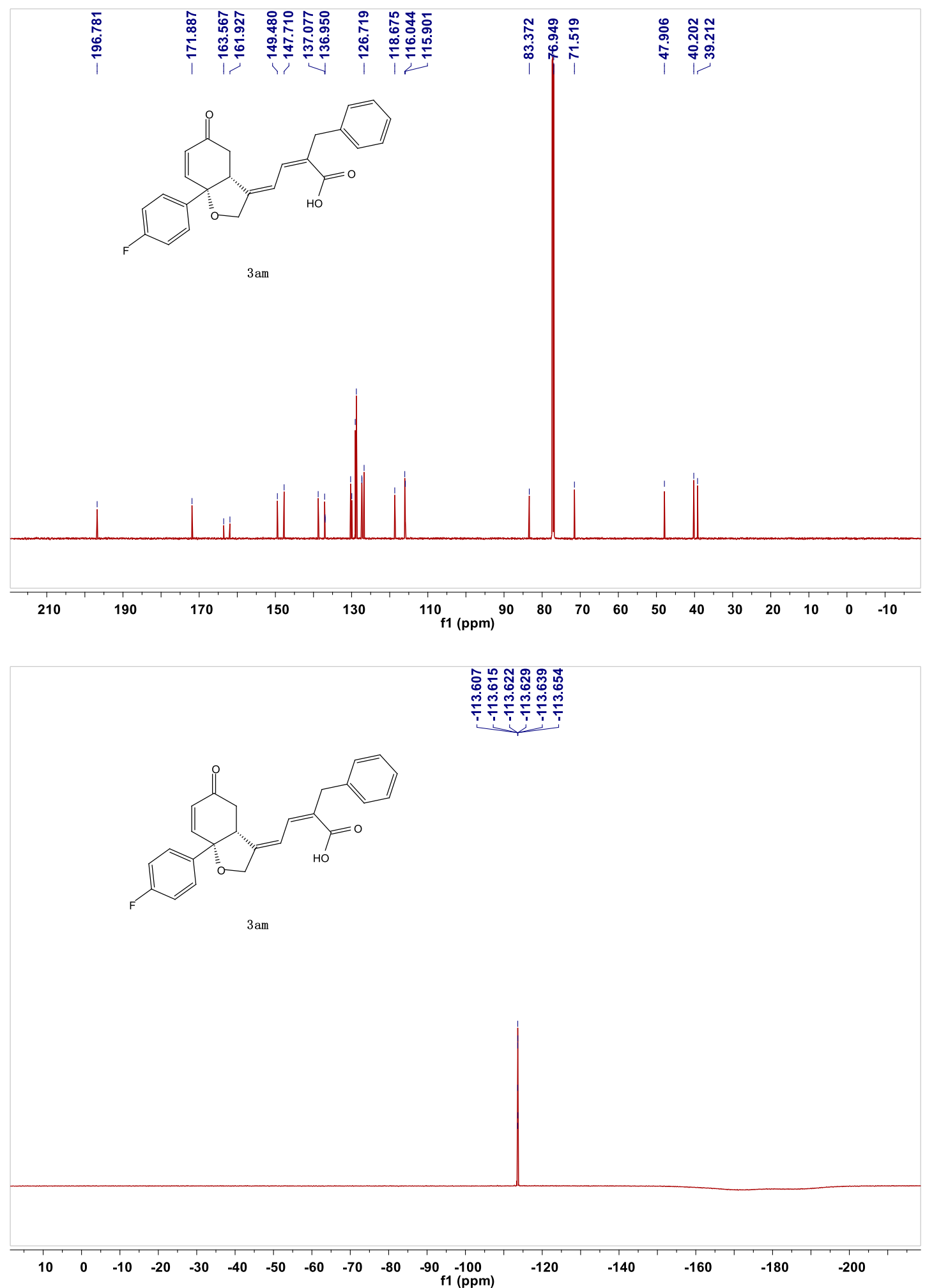

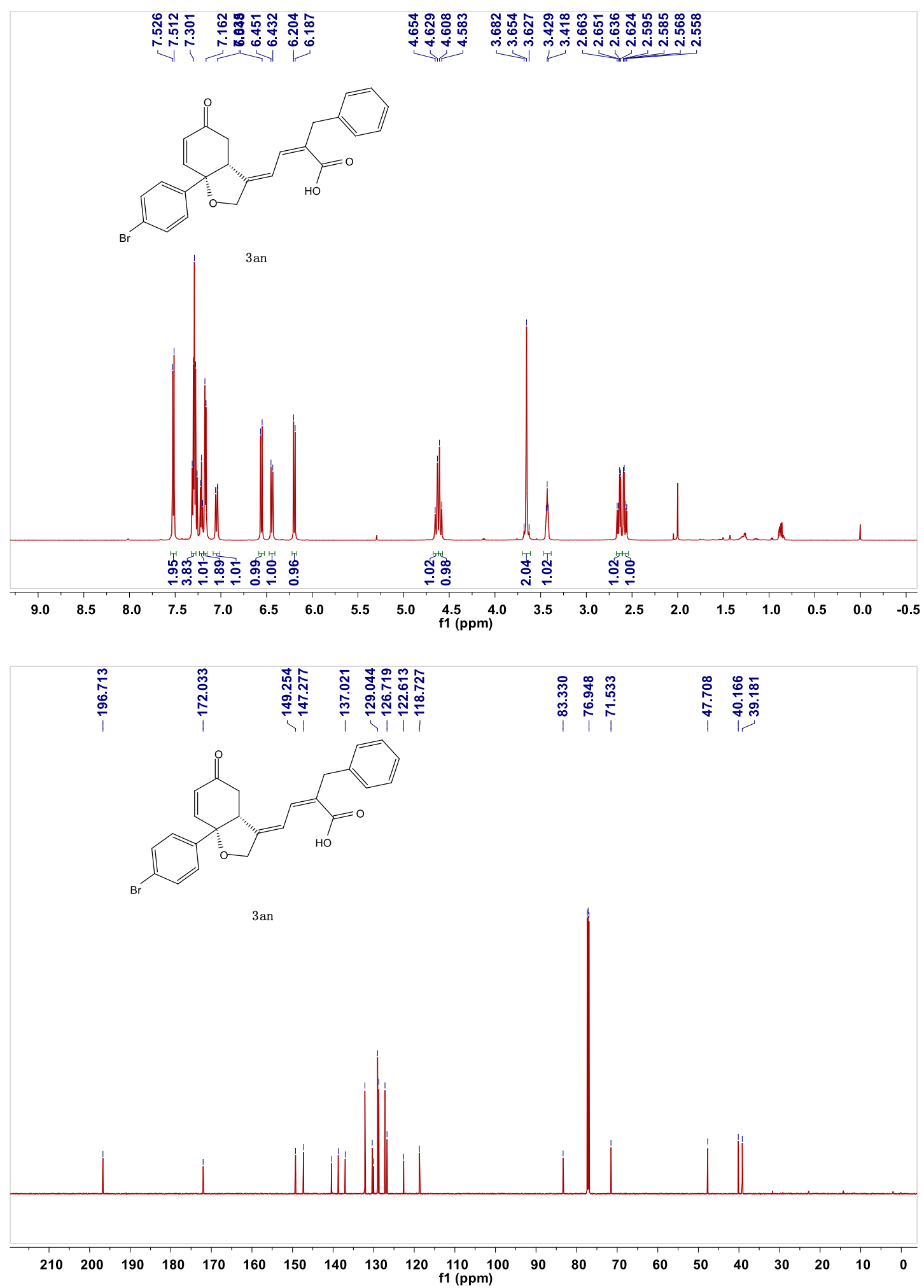

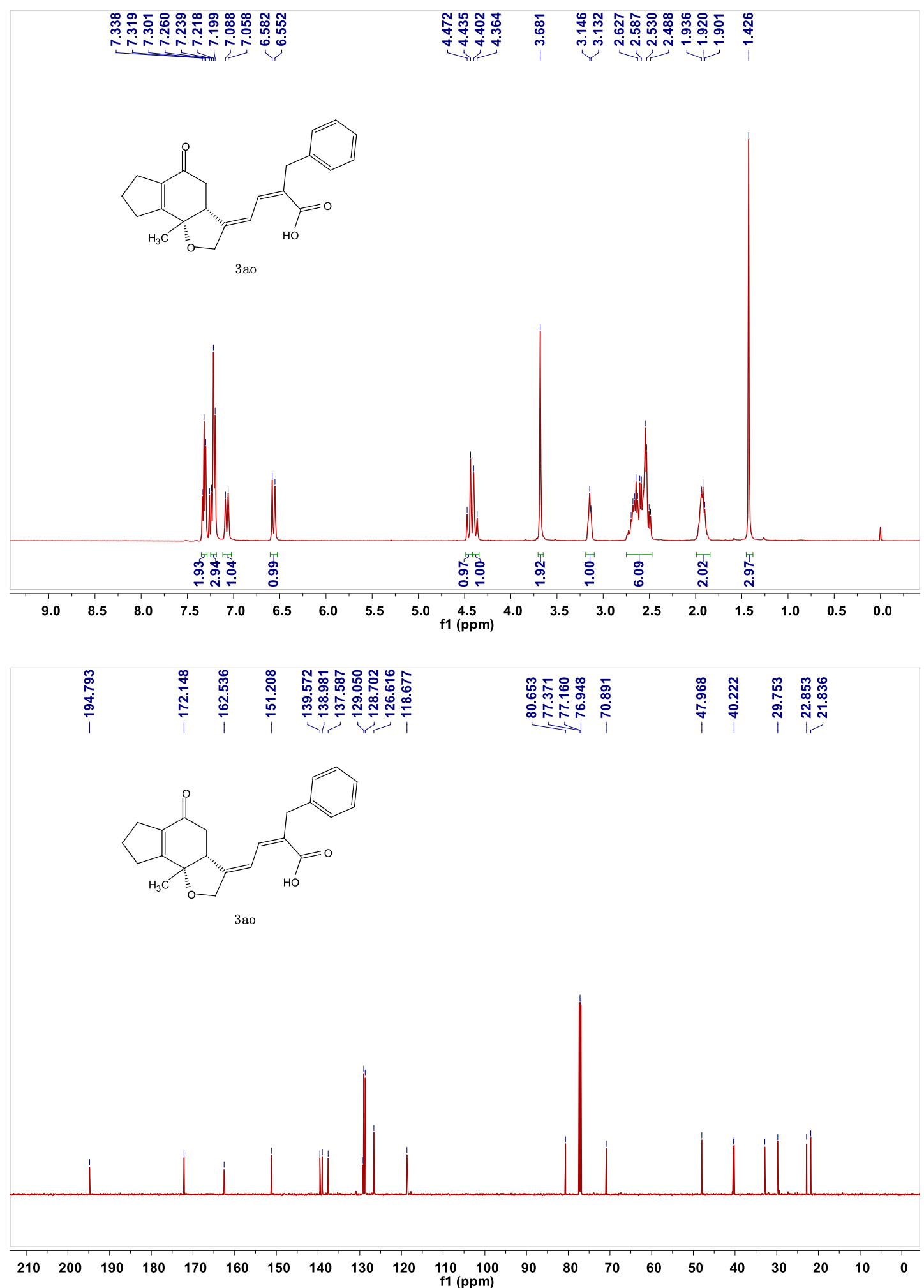

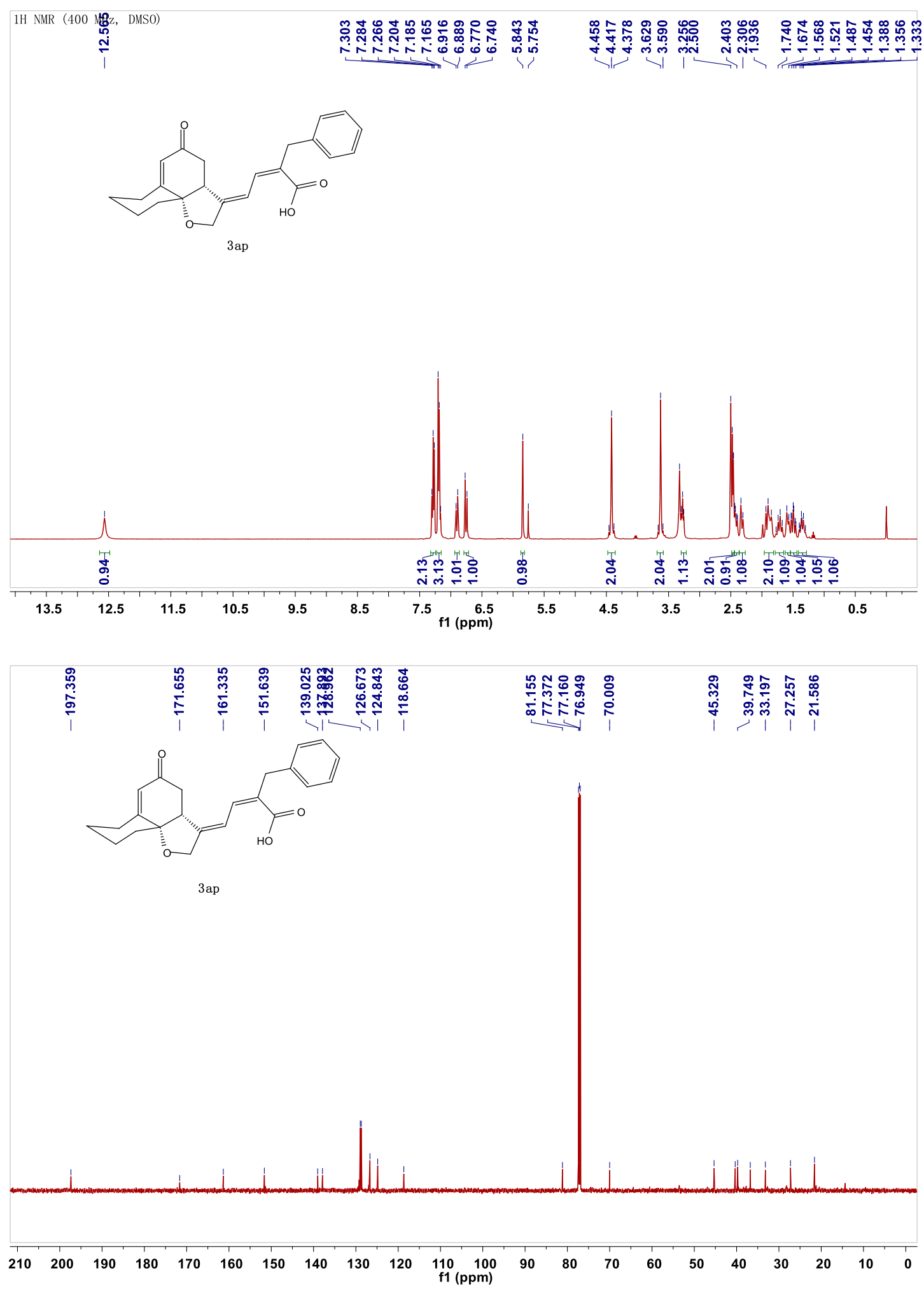

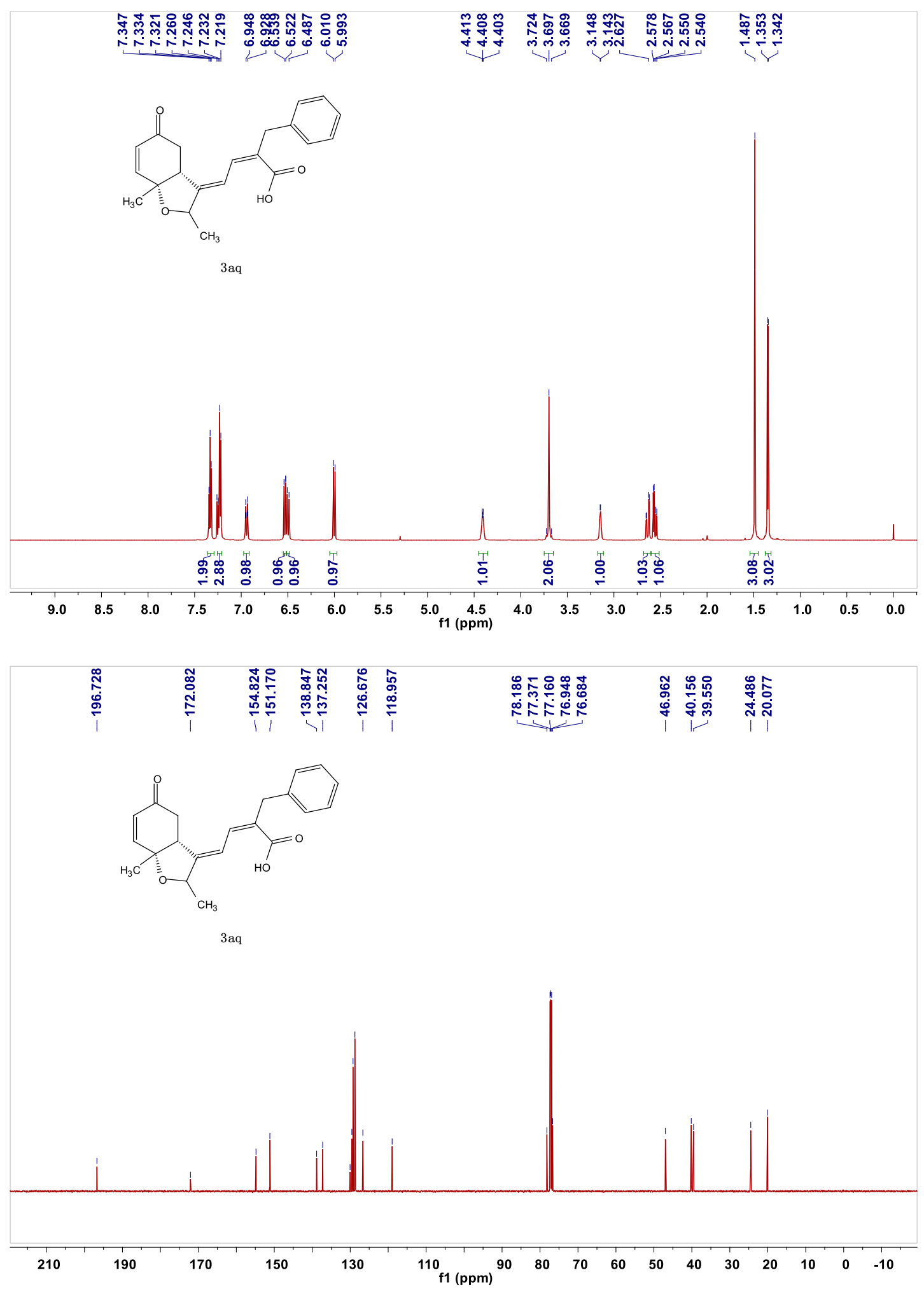

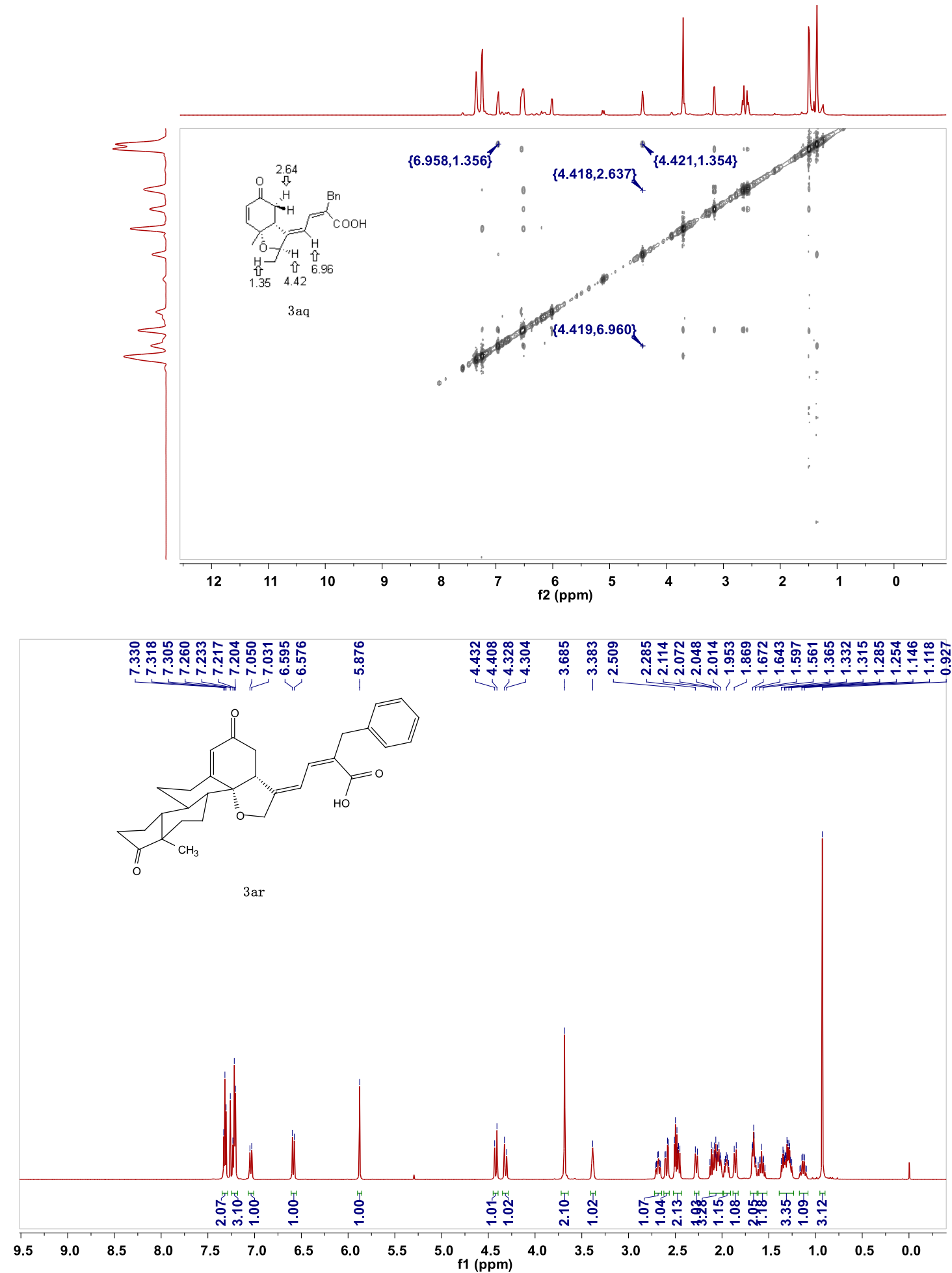


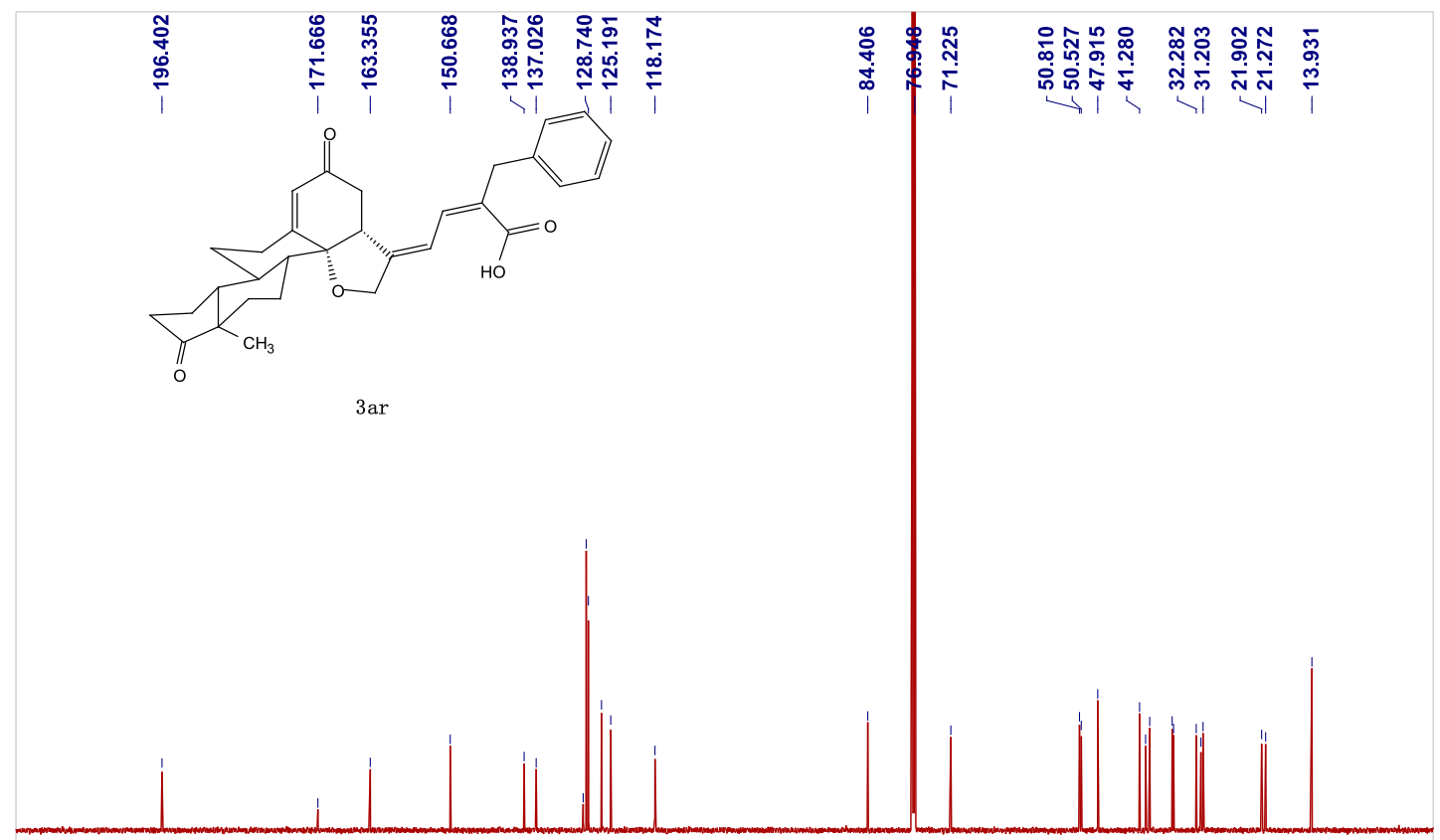

$\begin{array}{llllllllllllllllllllllllllll}210 & 200 & 190 & 180 & 170 & 160 & 150 & 140 & 130 & 120 & 110 & 100 & 90 & 80 & 70 & 60 & 50 & 40 & 30 & 20 & 10 & 0\end{array}$

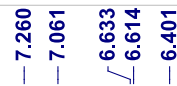

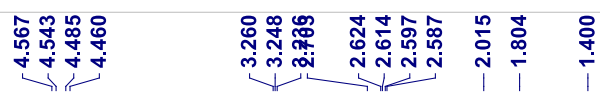

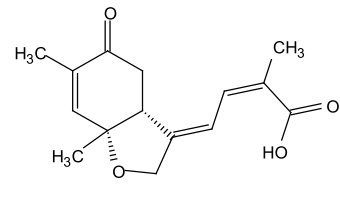

$3 \mathrm{bb}$

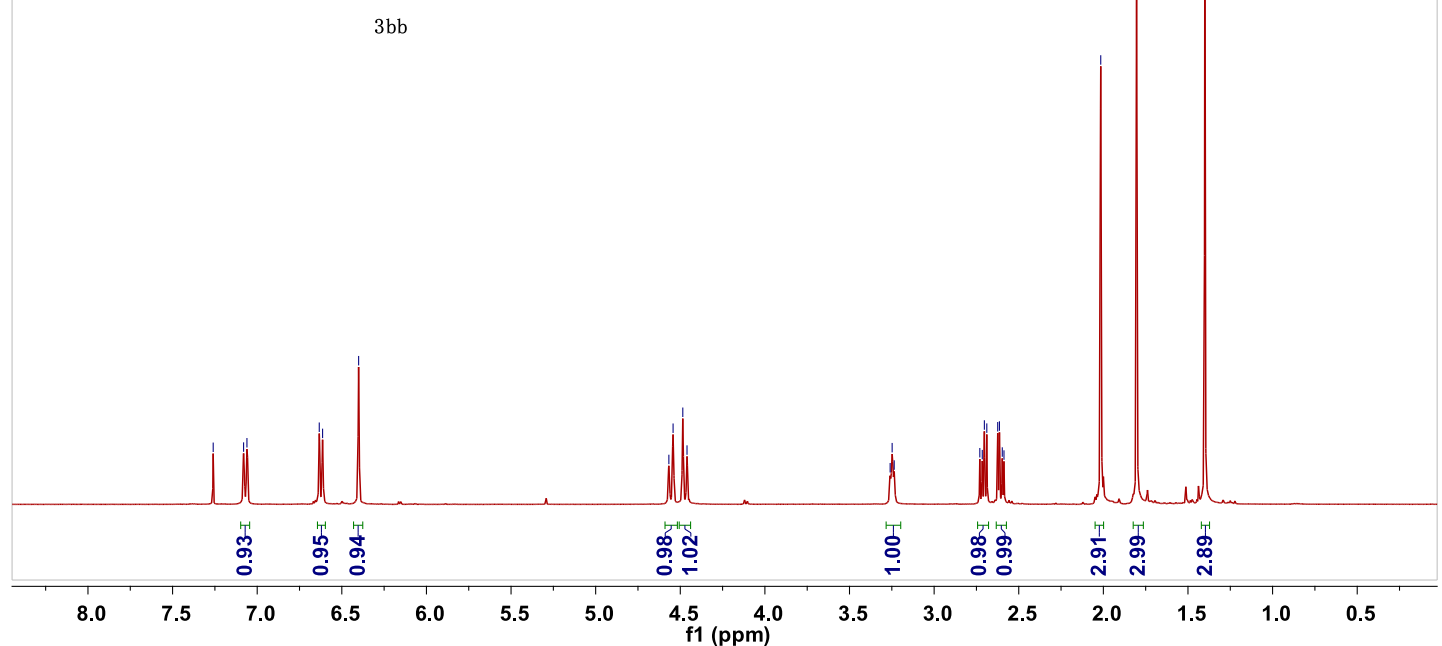



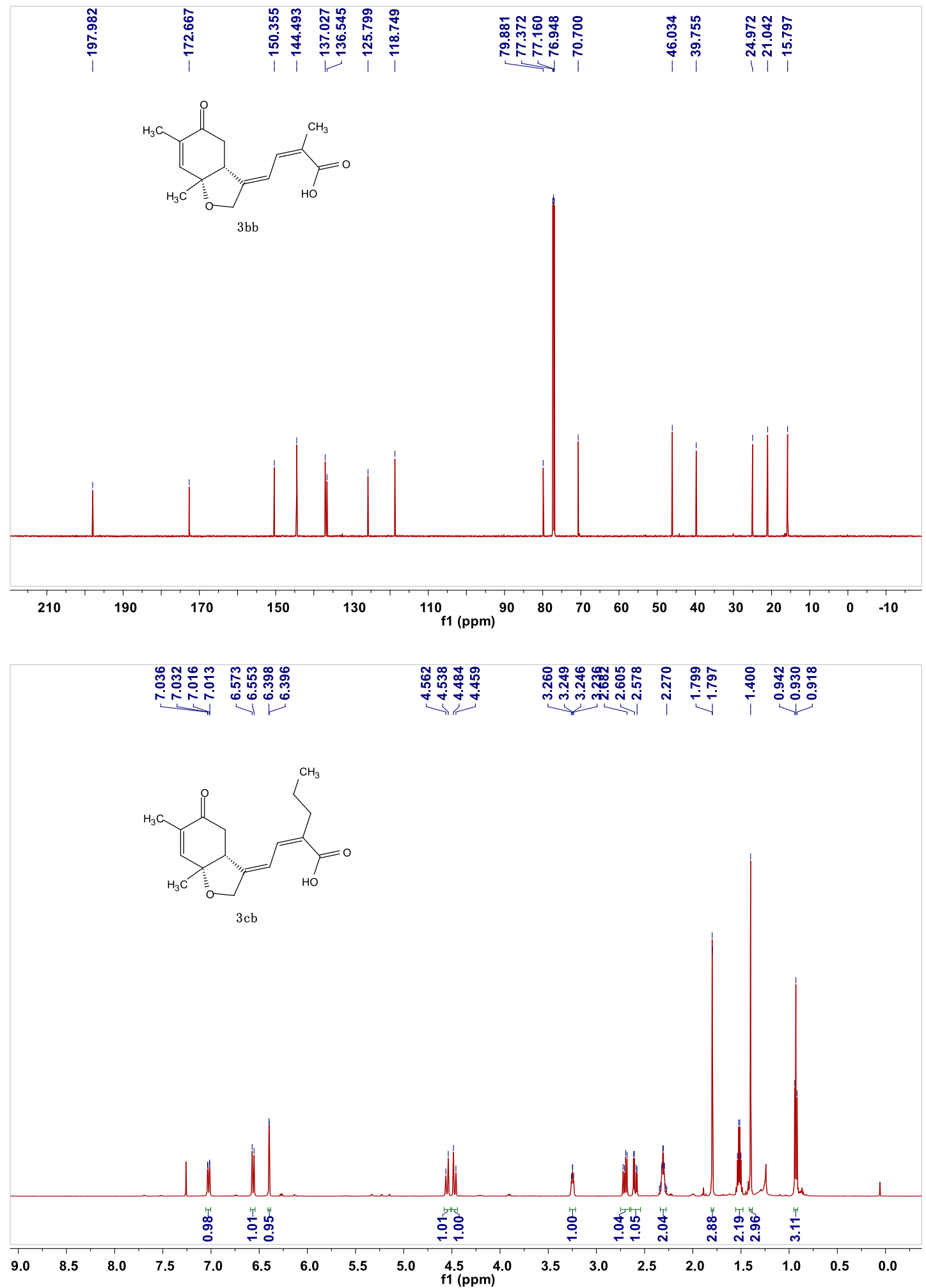

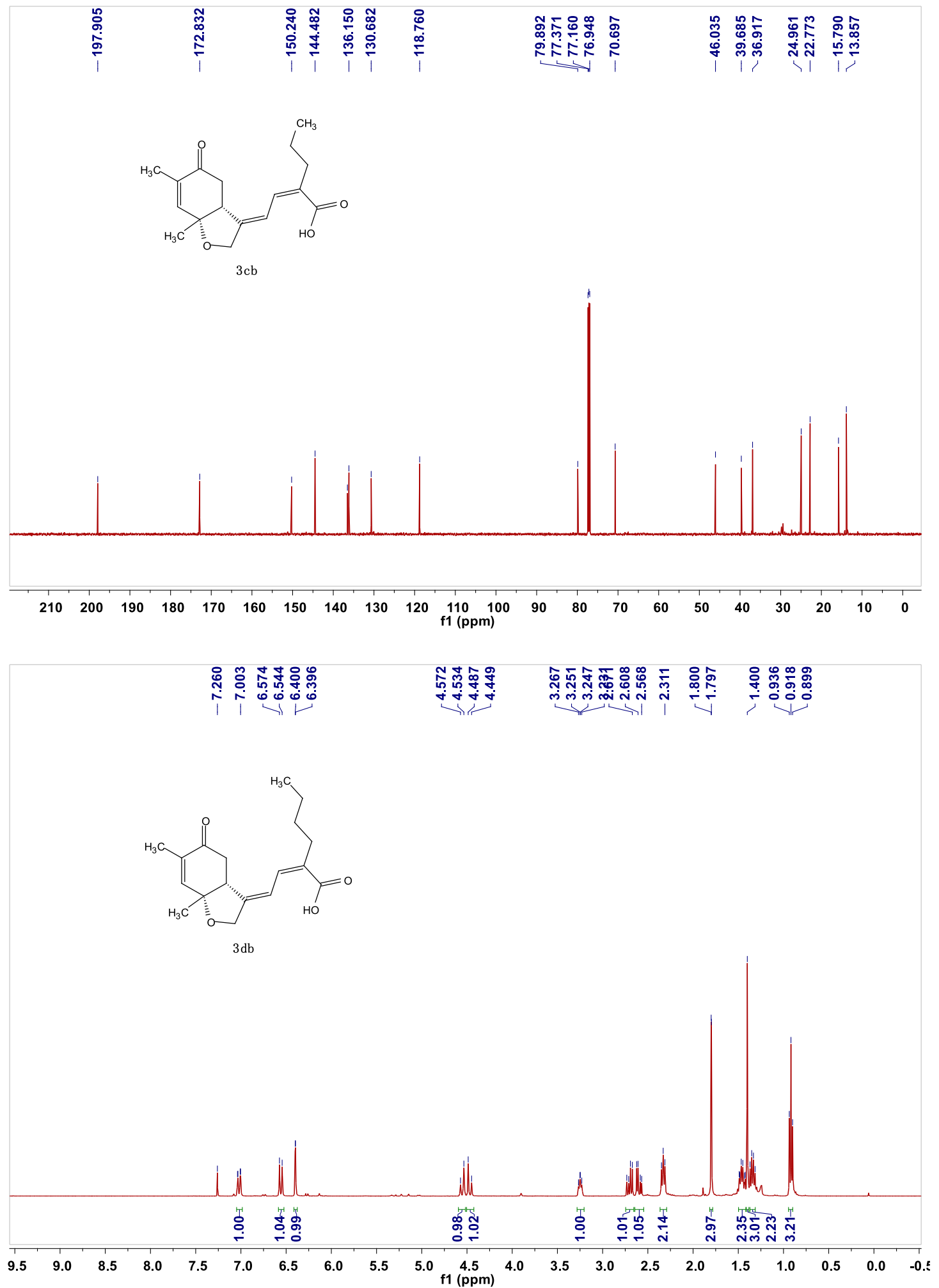


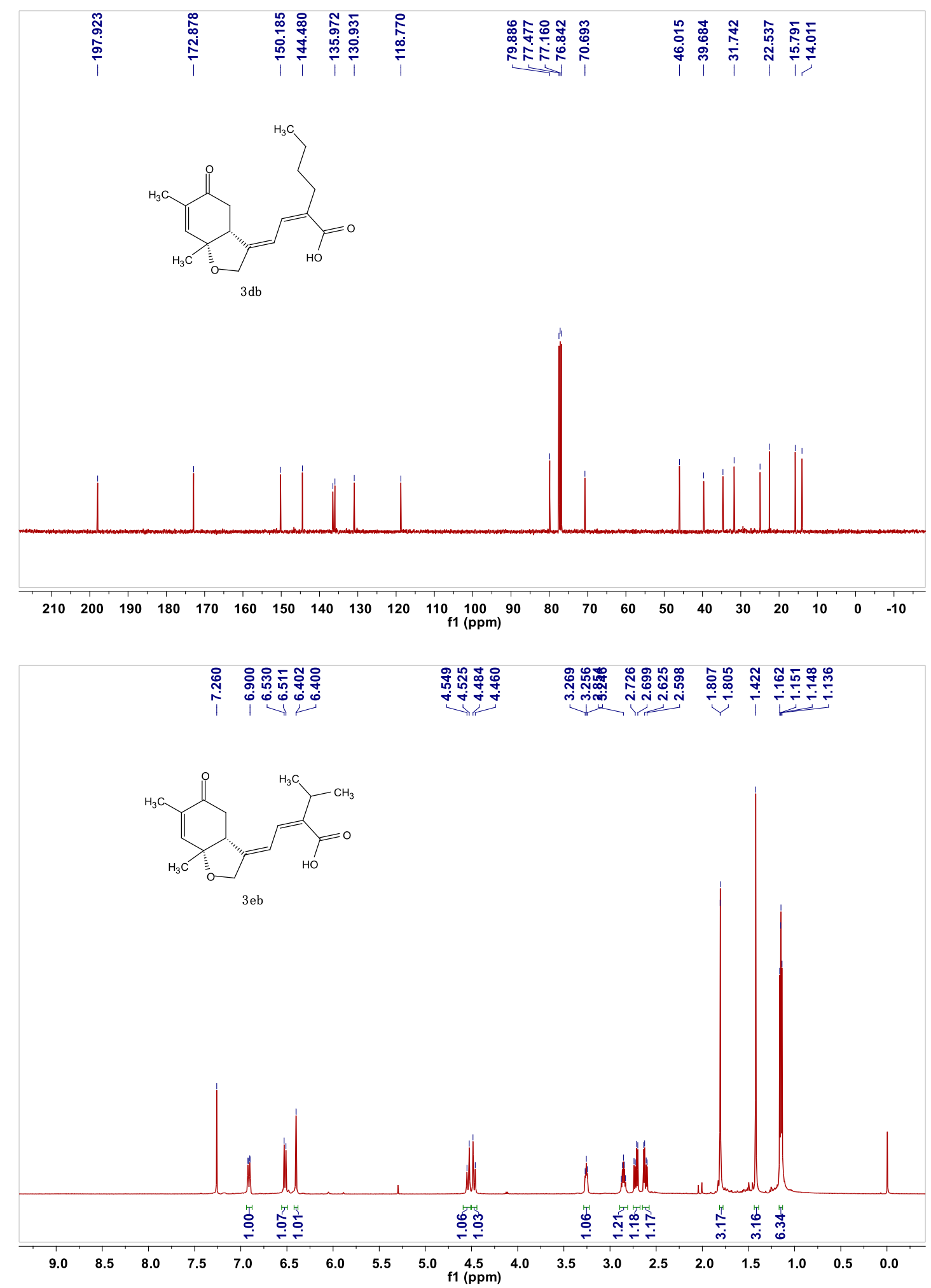




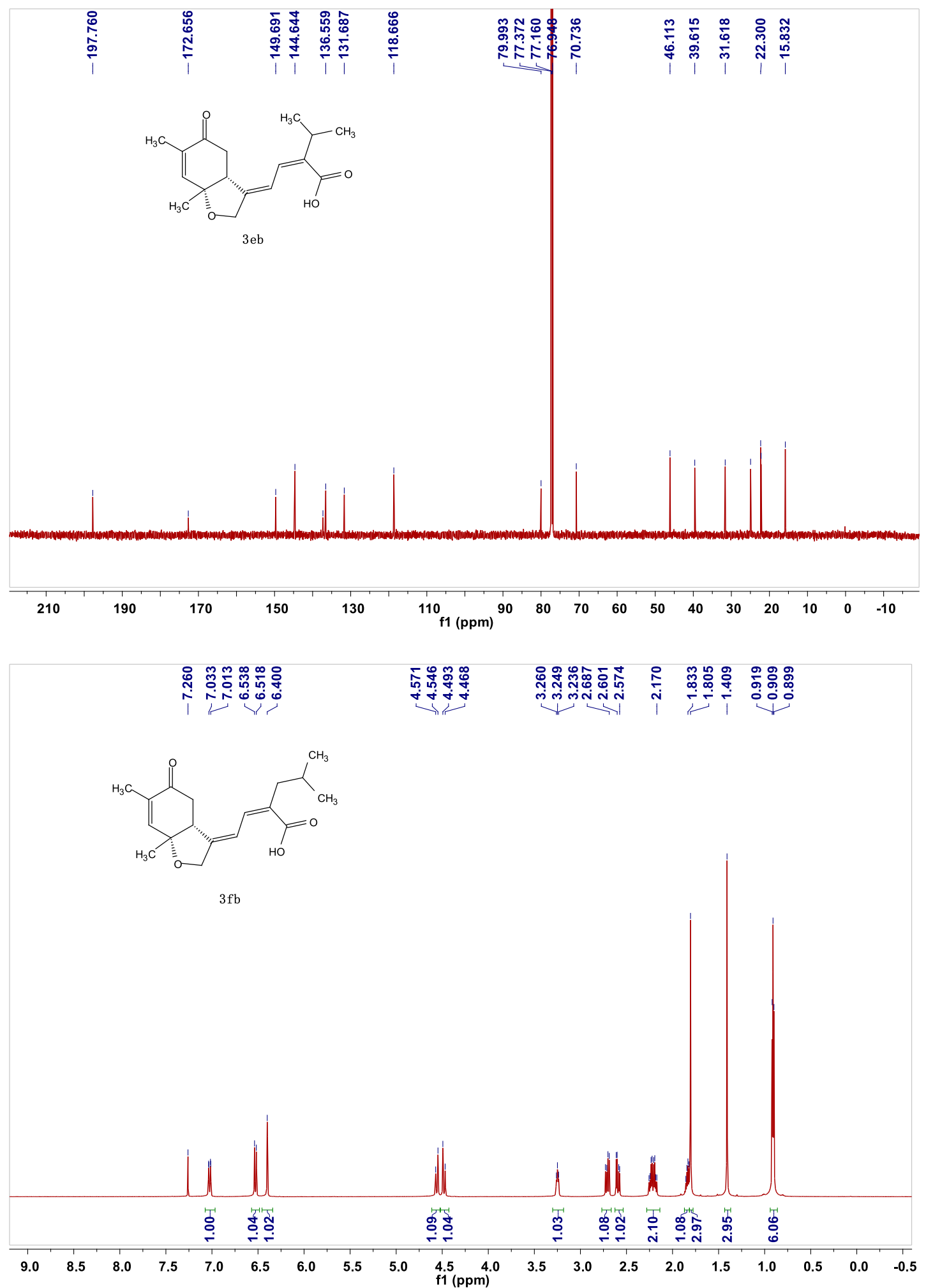



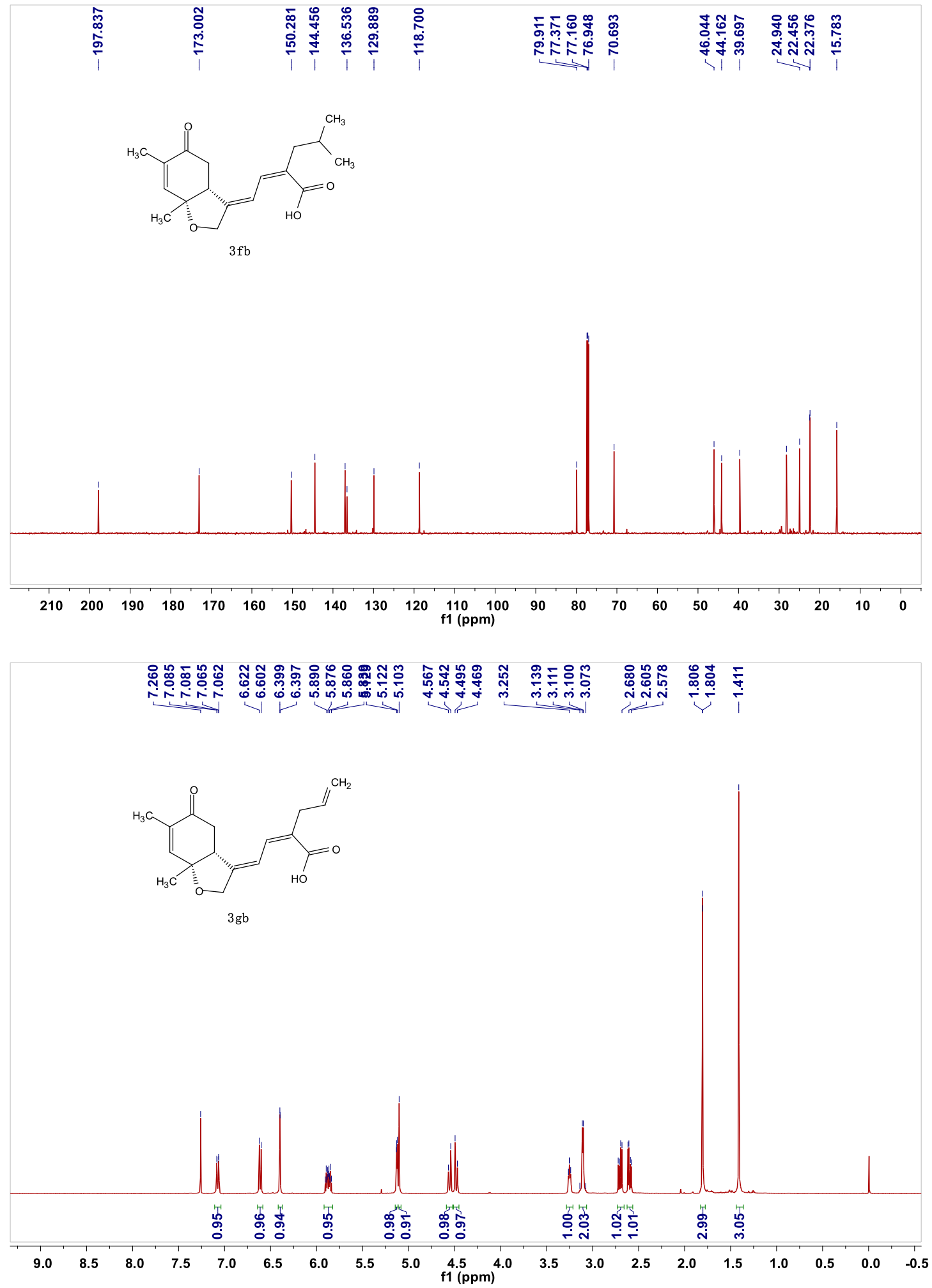


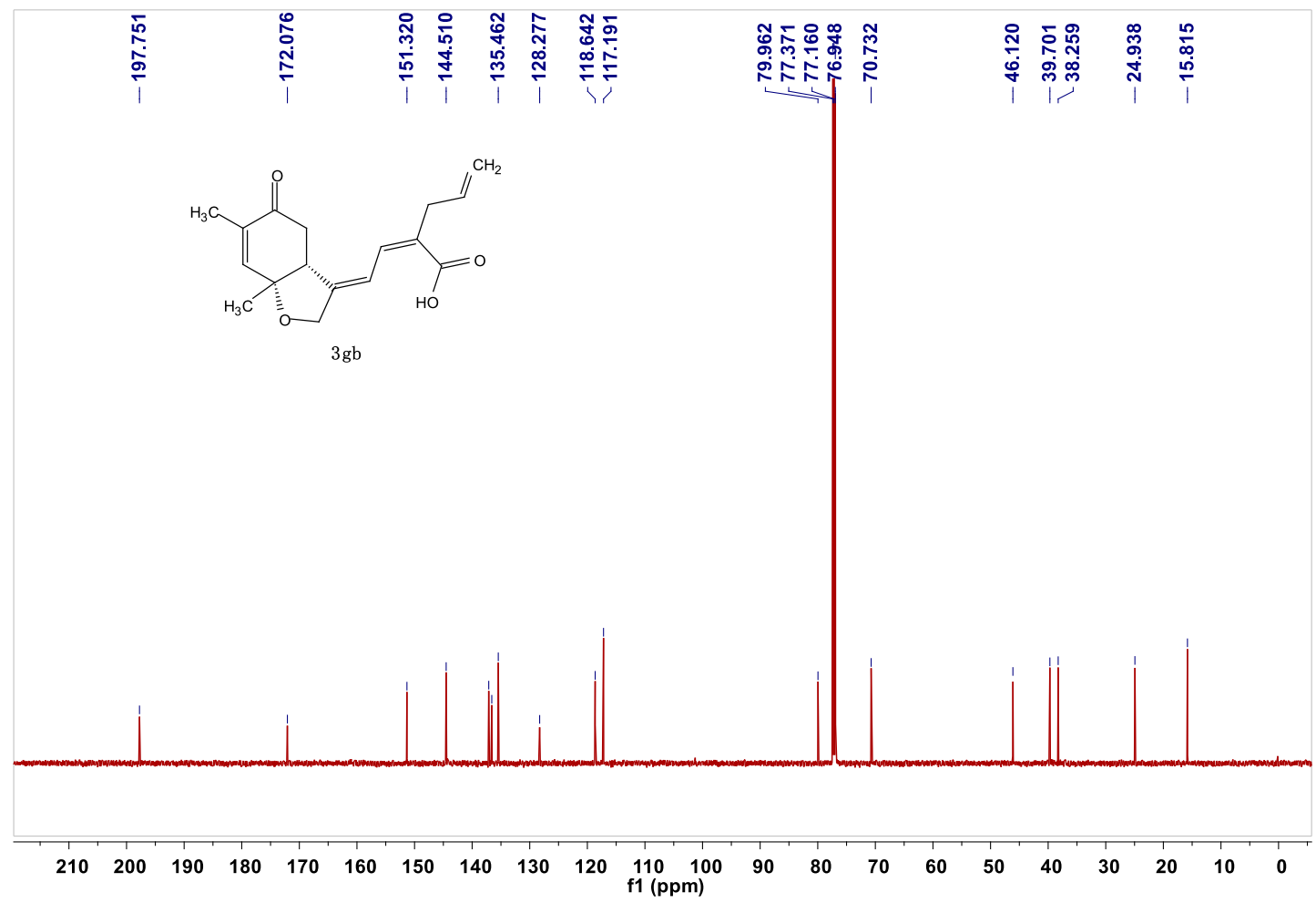

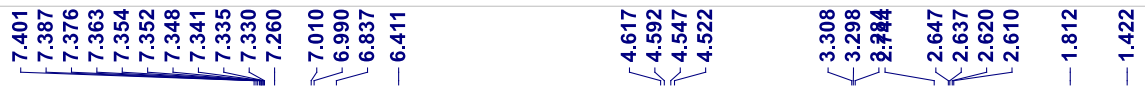

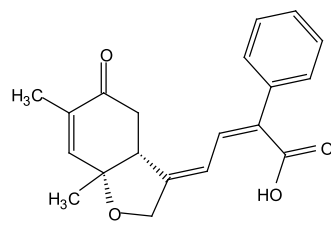

$3 \mathrm{hb}$

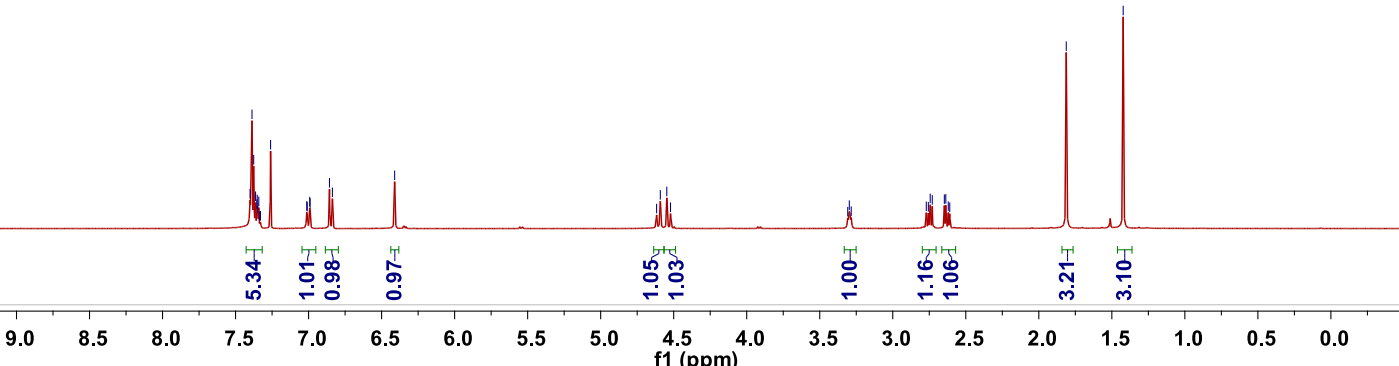



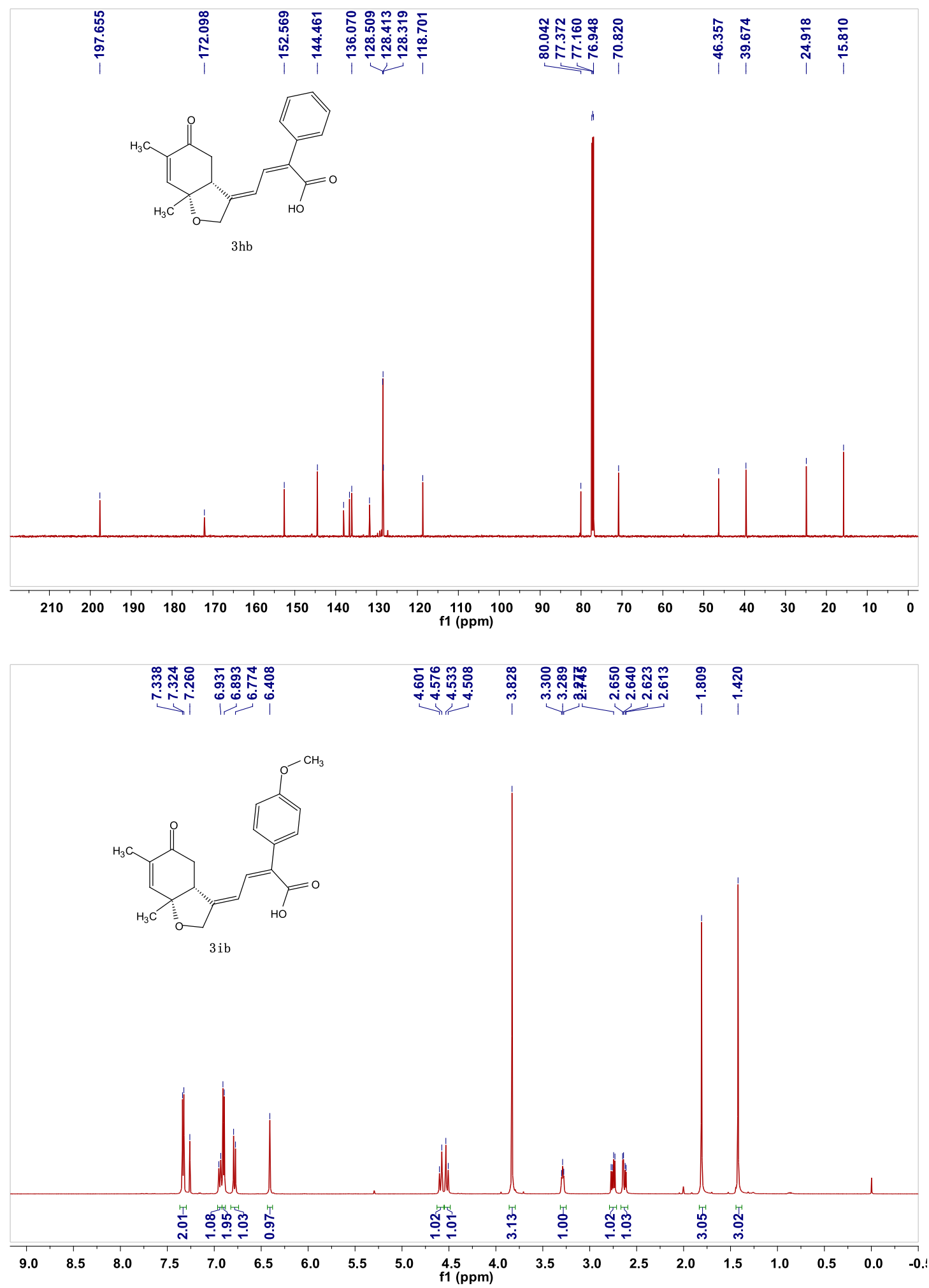

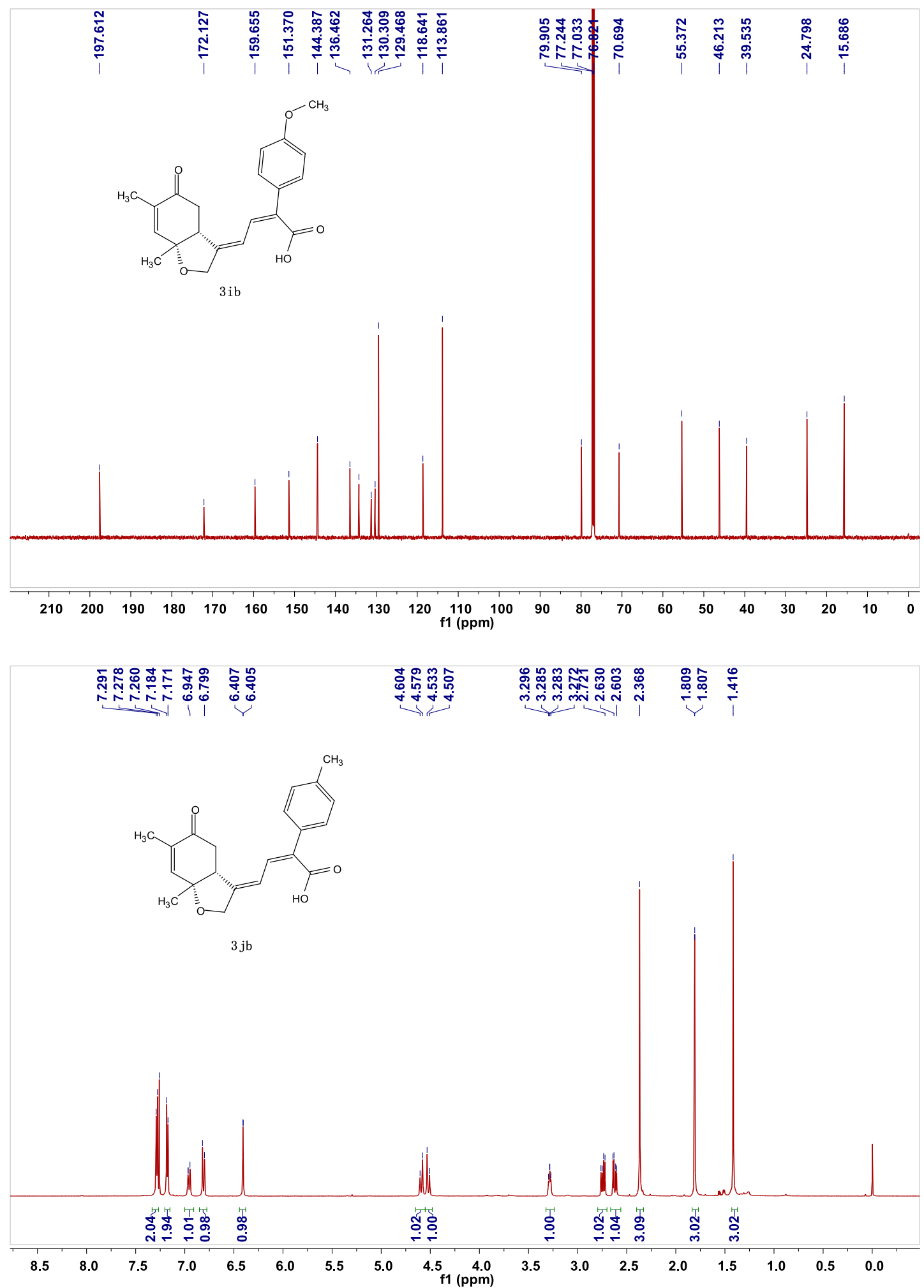


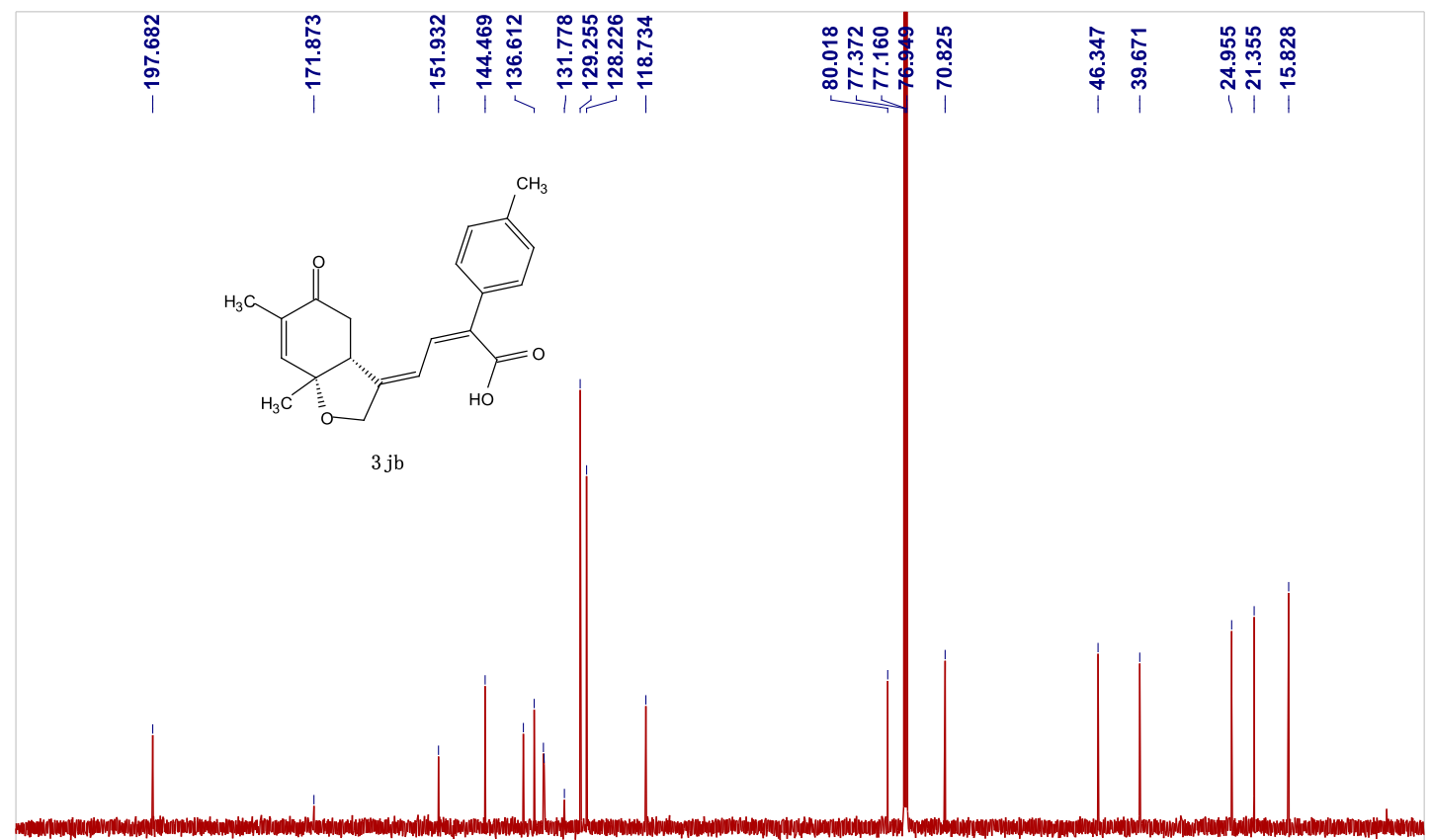

$\begin{array}{lllllllllllllllllllll}210 & 200 & 190 & 180 & 170 & 160 & 150 & 140 & 130 & 120 & \begin{array}{c}110 \\ \mathrm{f} 1(\mathrm{ppm})\end{array} & 90 & 80 & 70 & 60 & 50 & 40 & 30 & 20 & 10 & 0\end{array}$

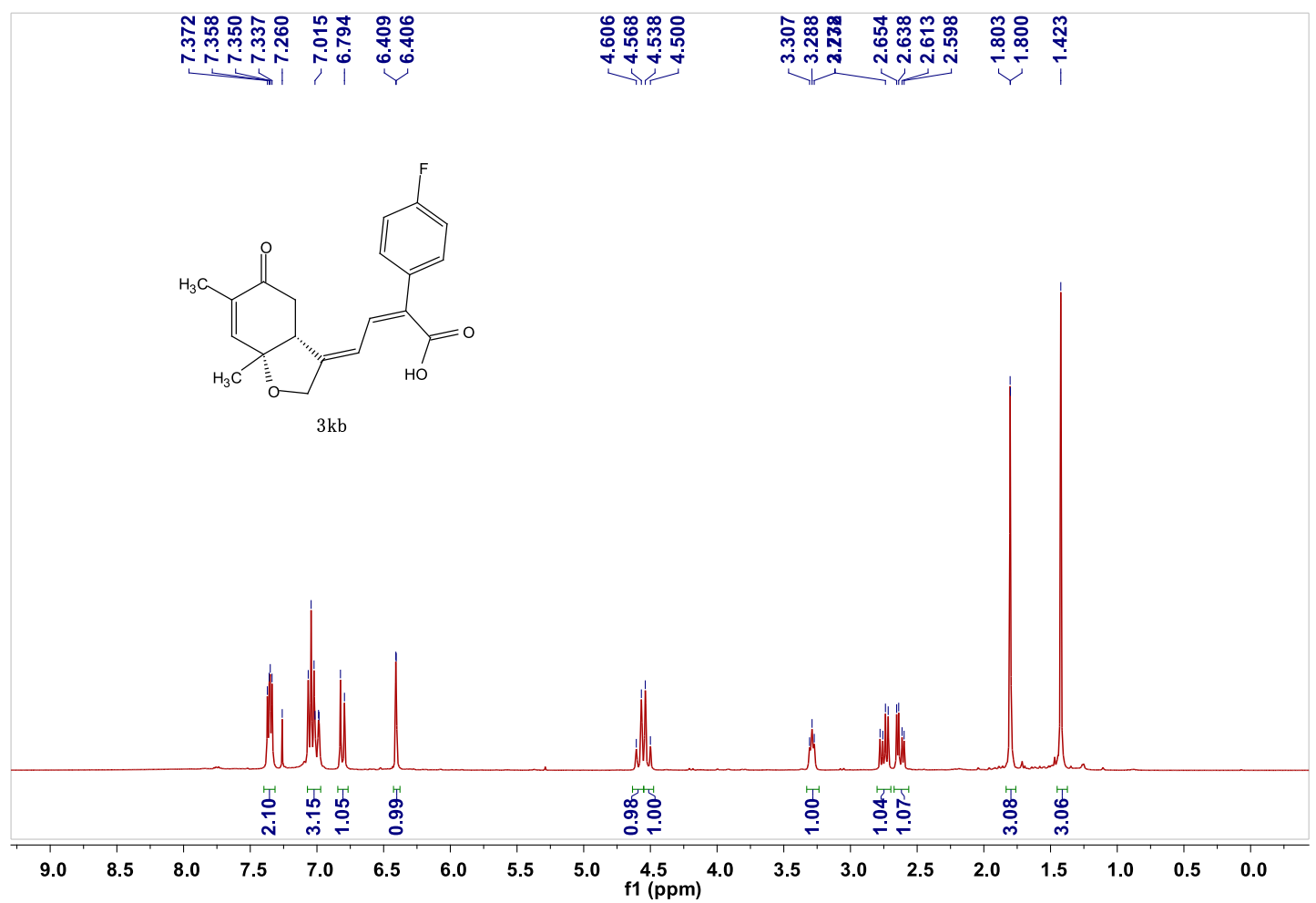



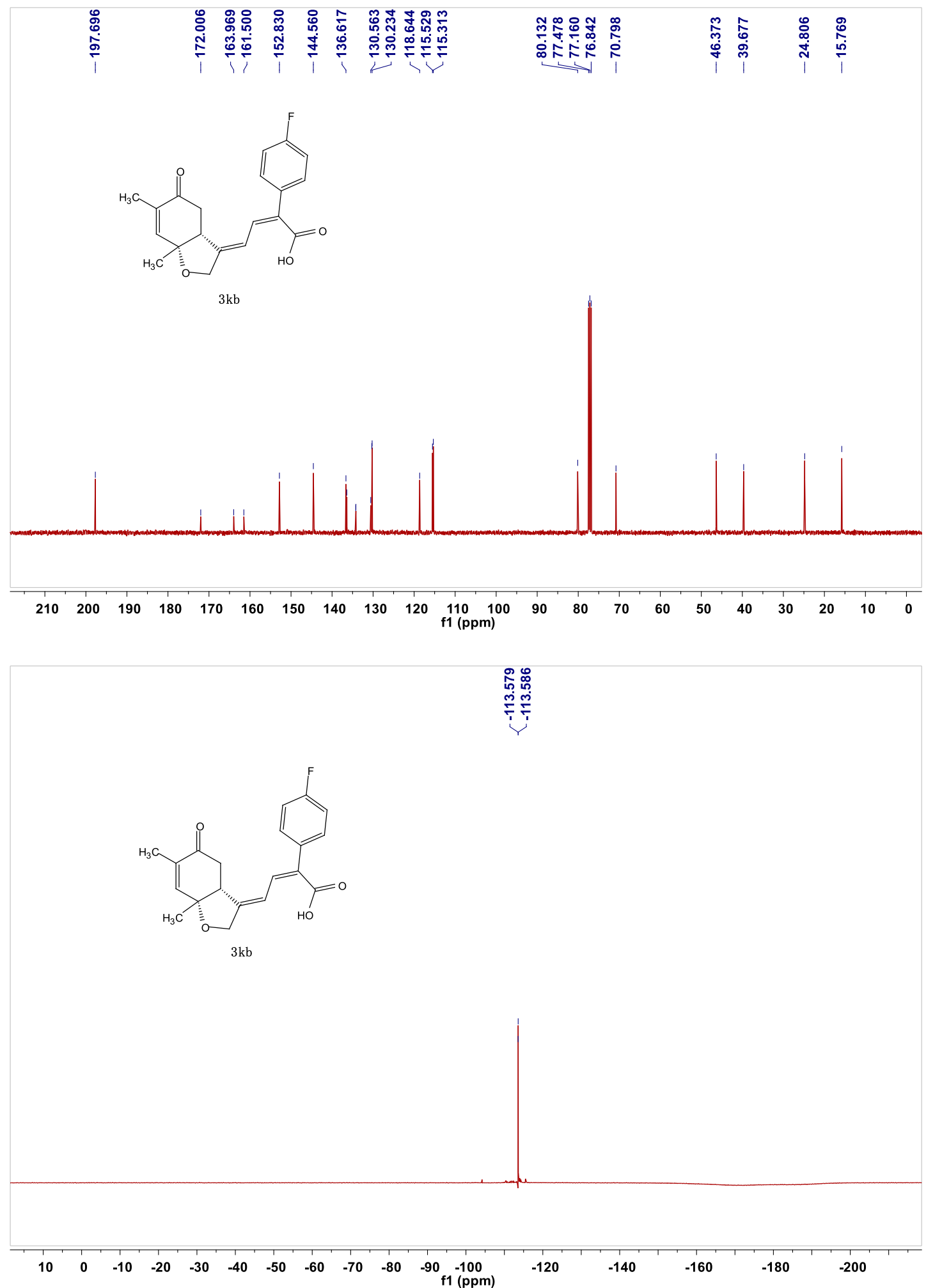

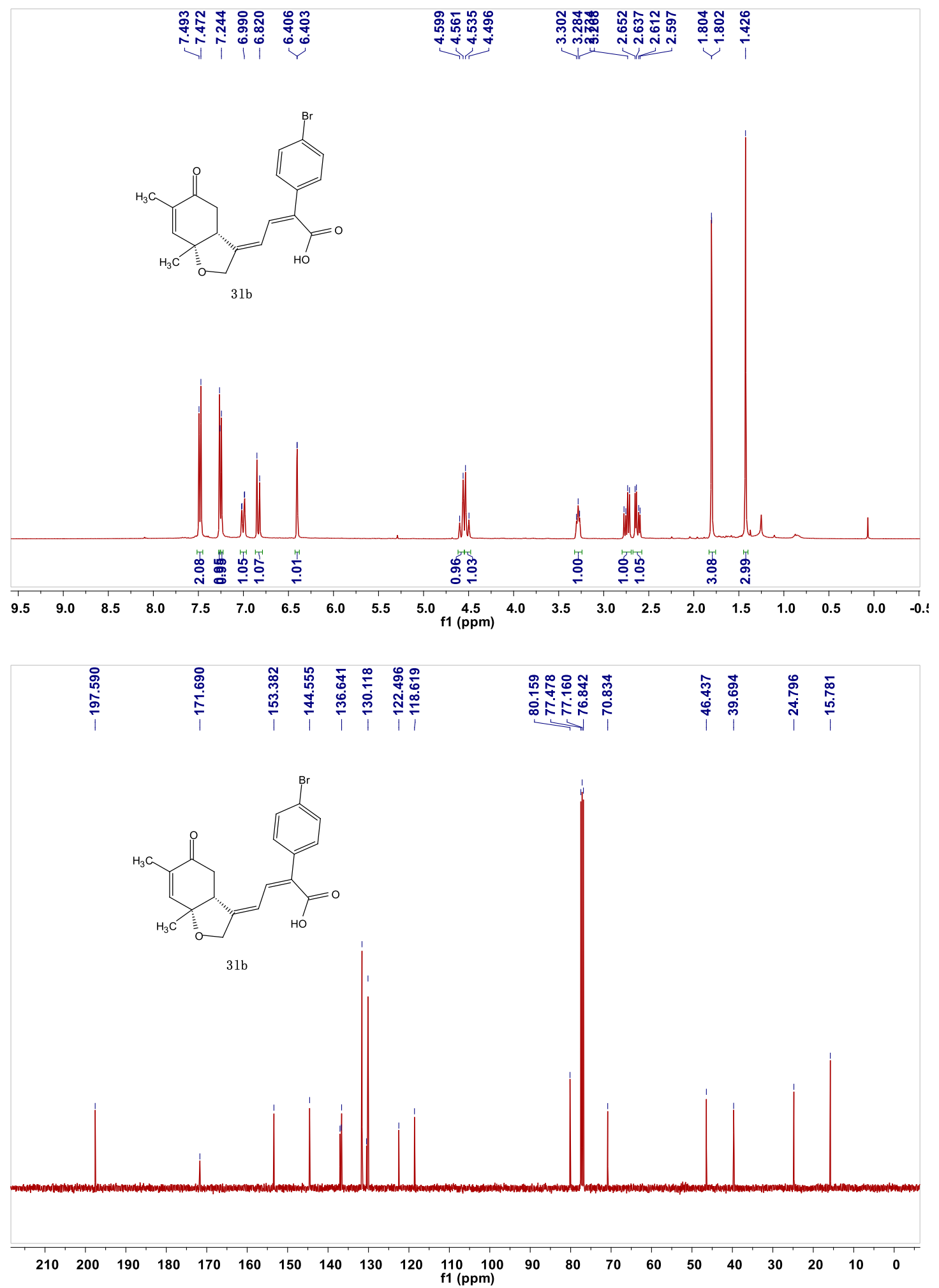

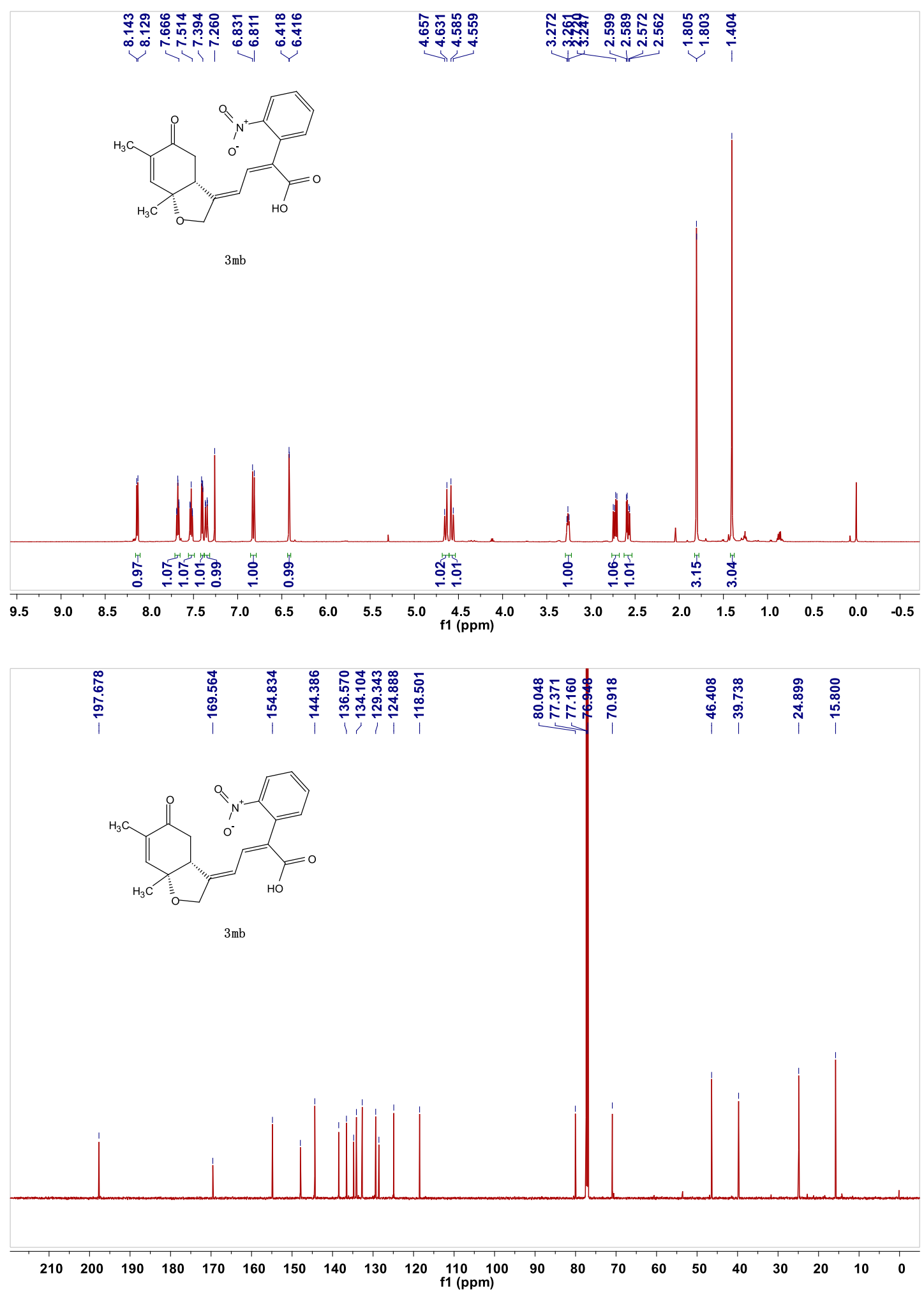

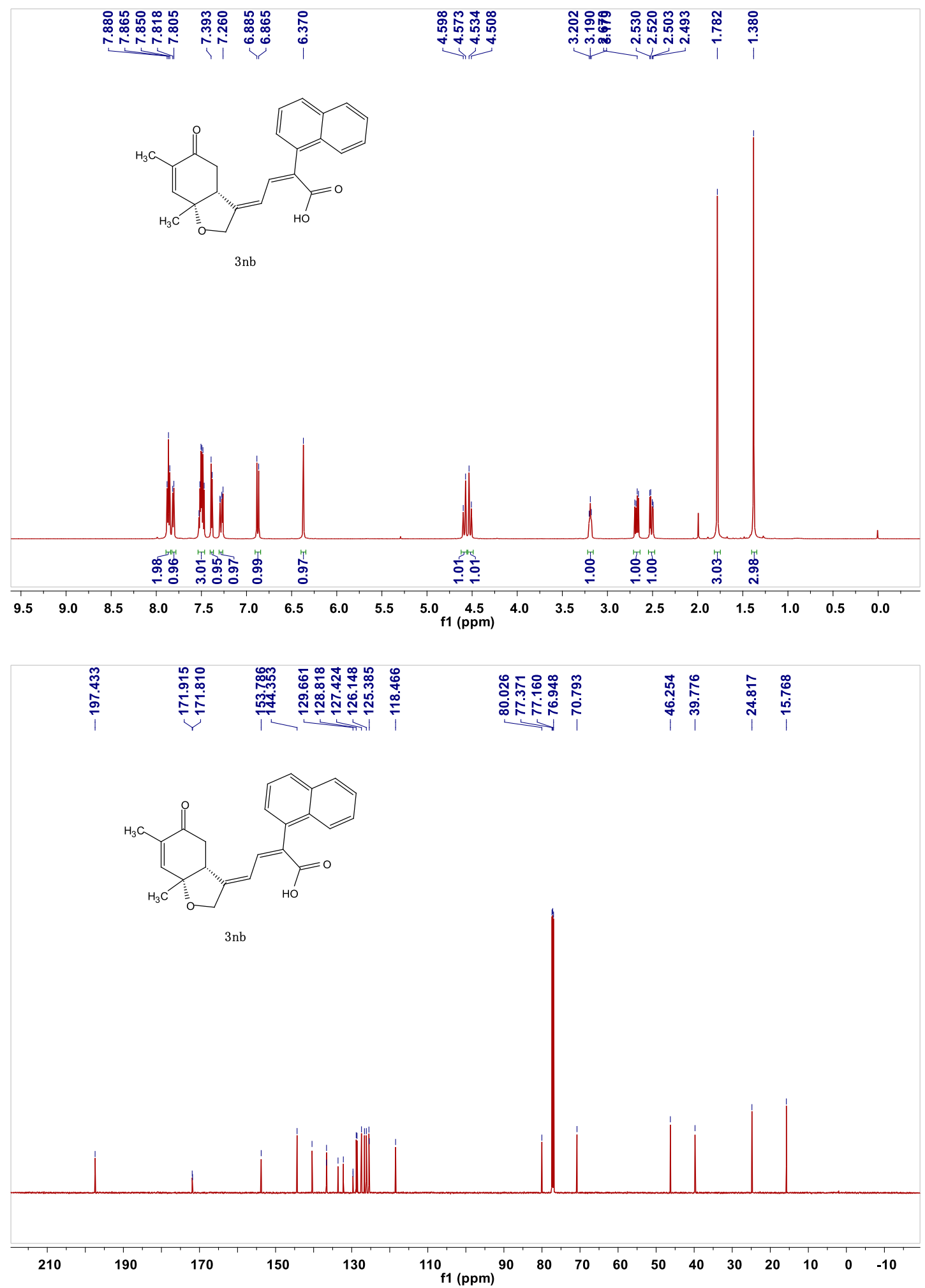

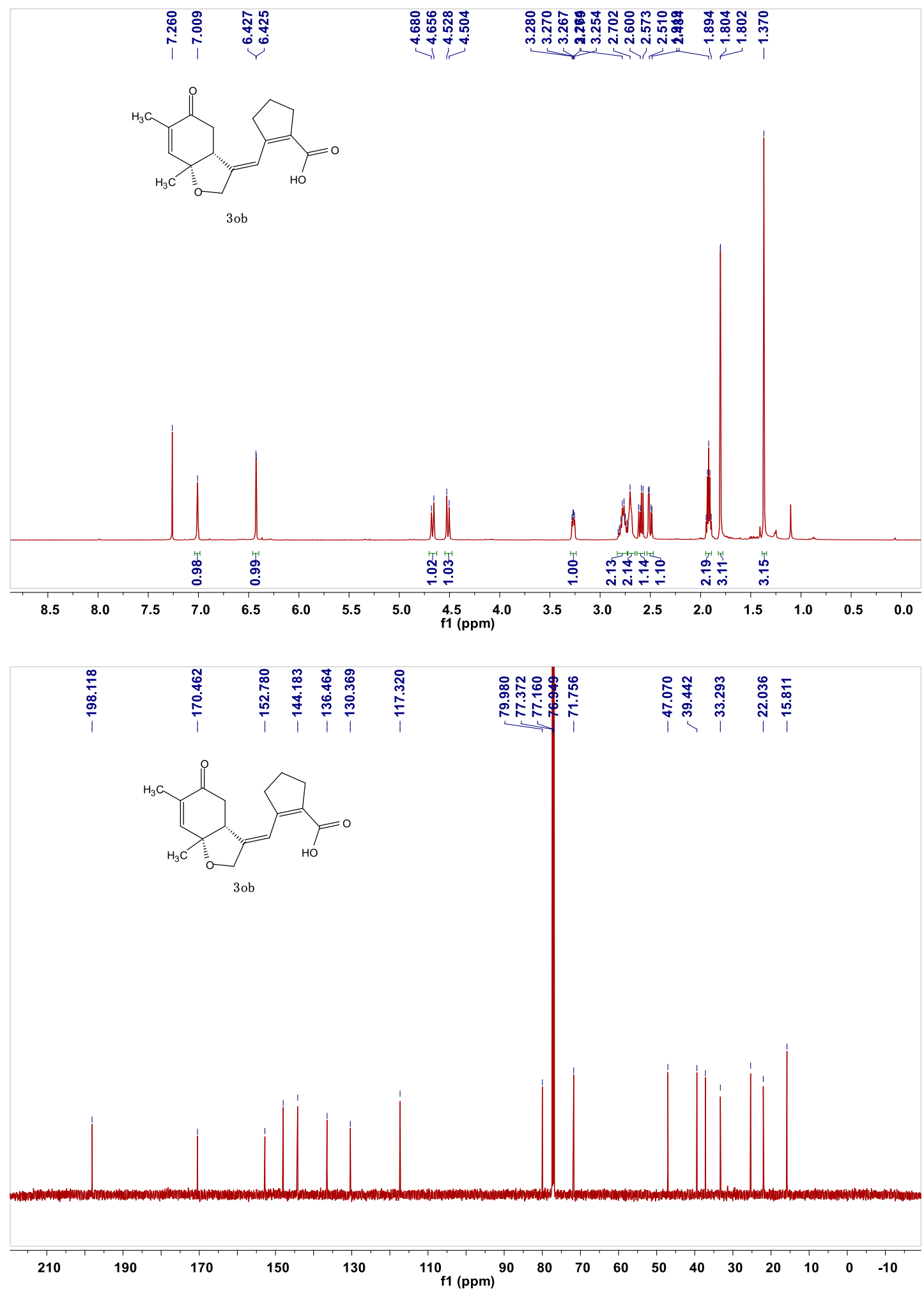

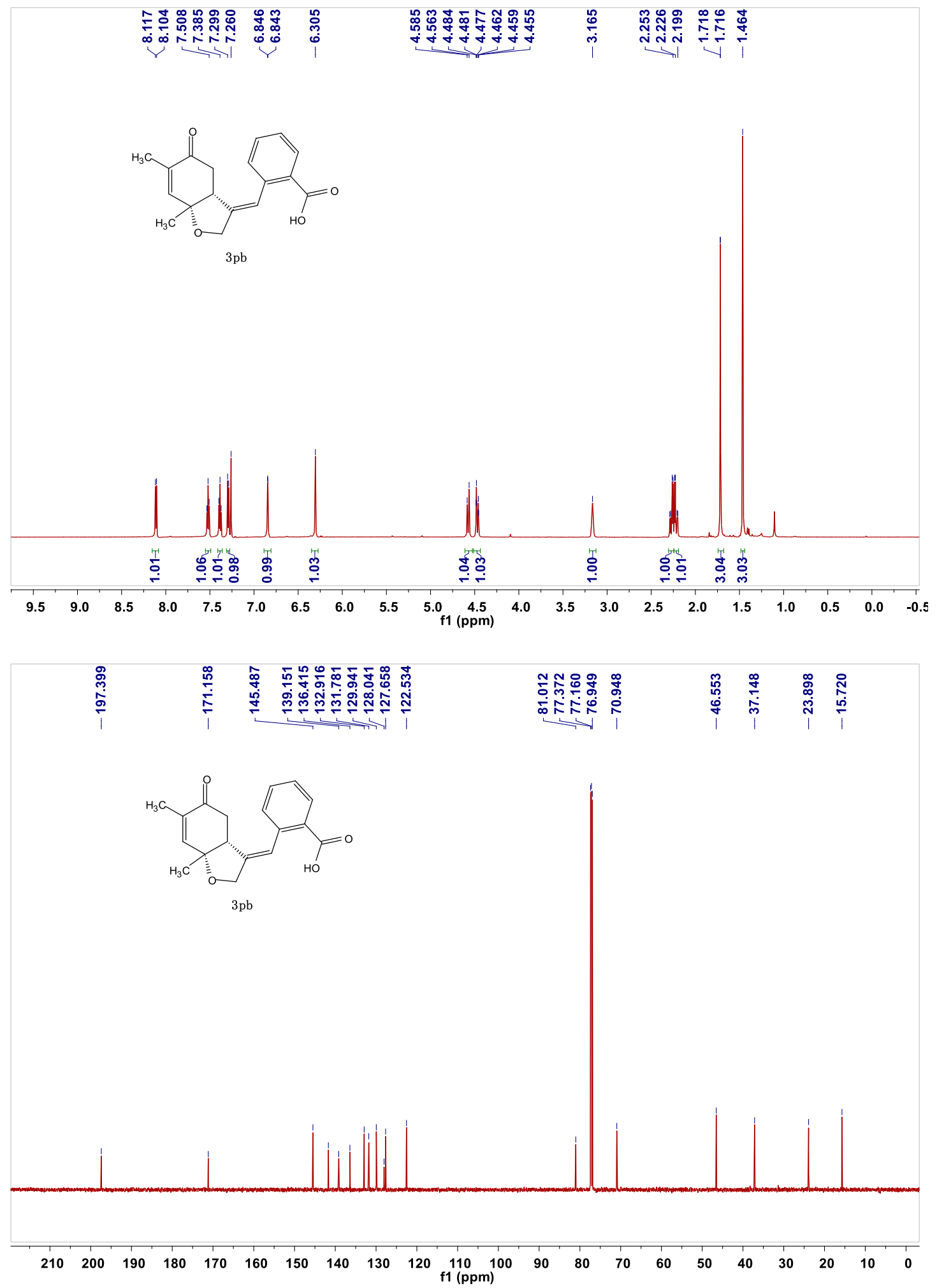

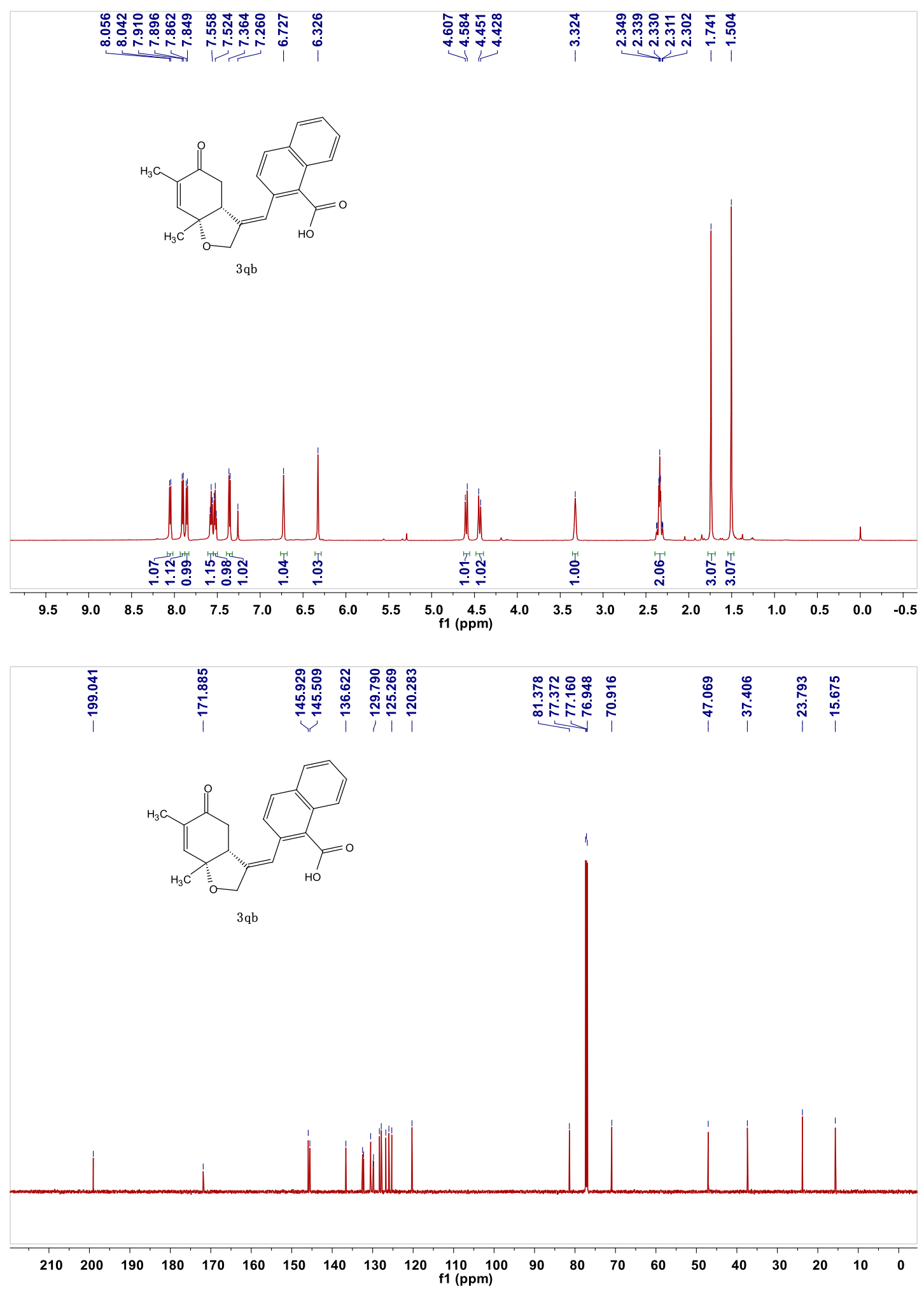

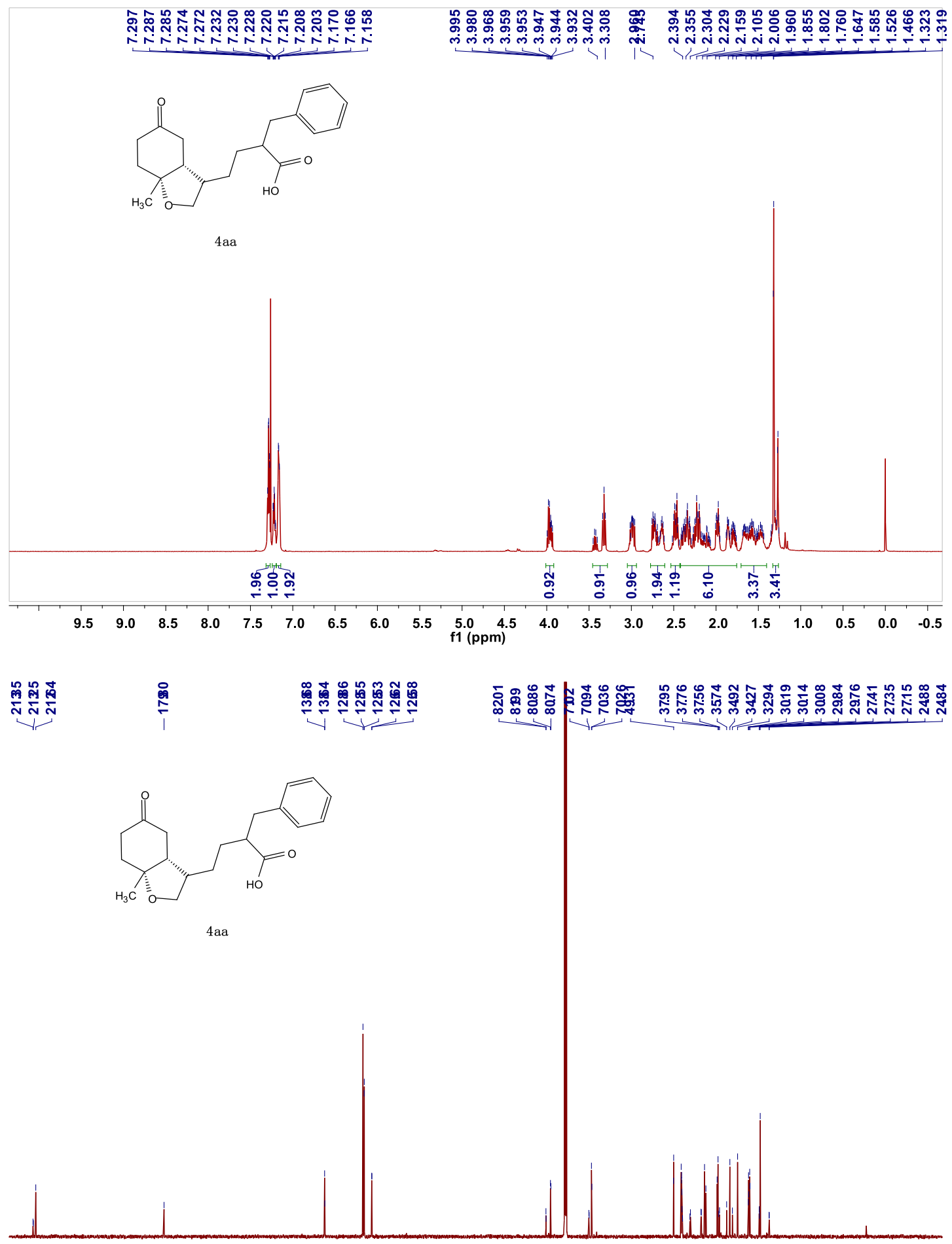

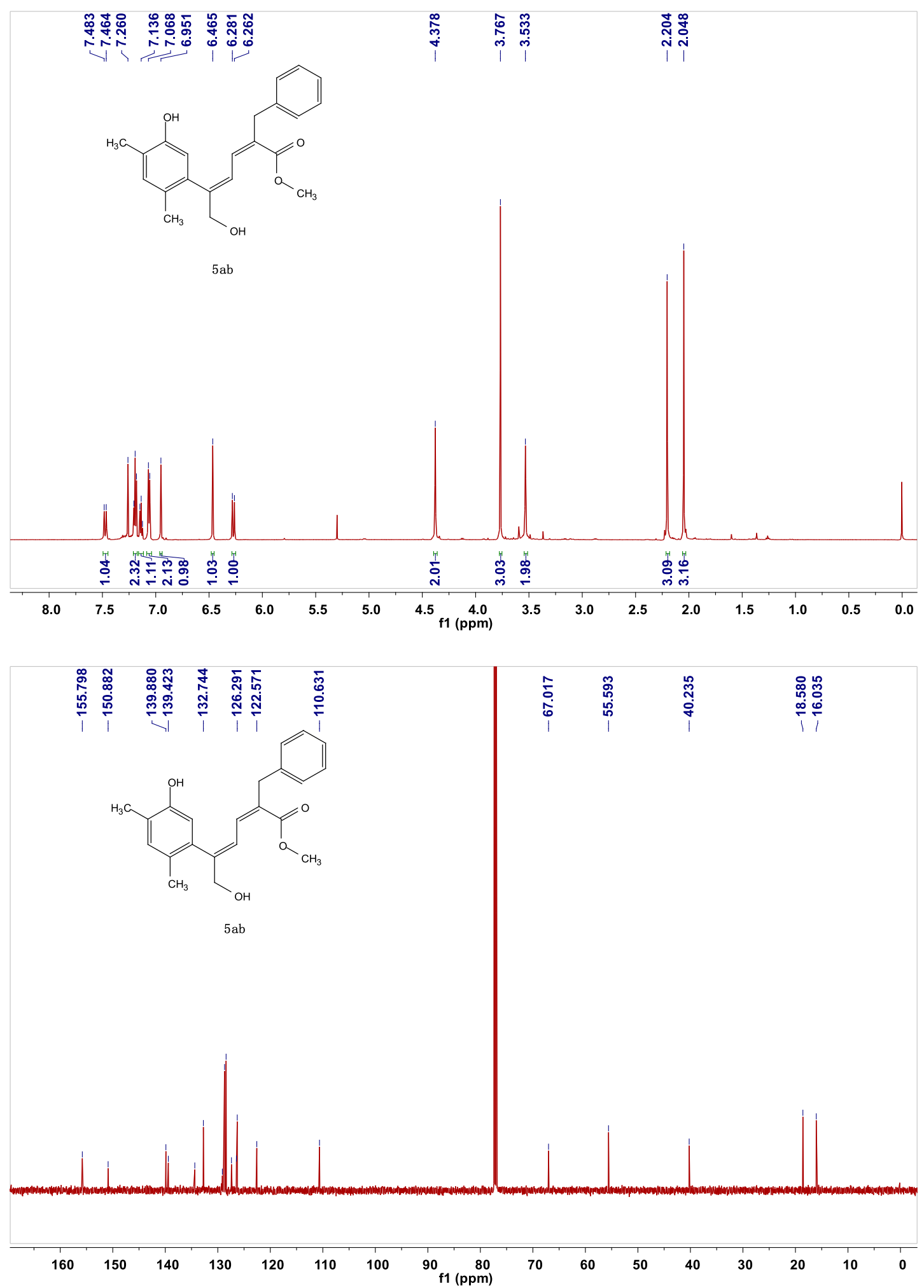

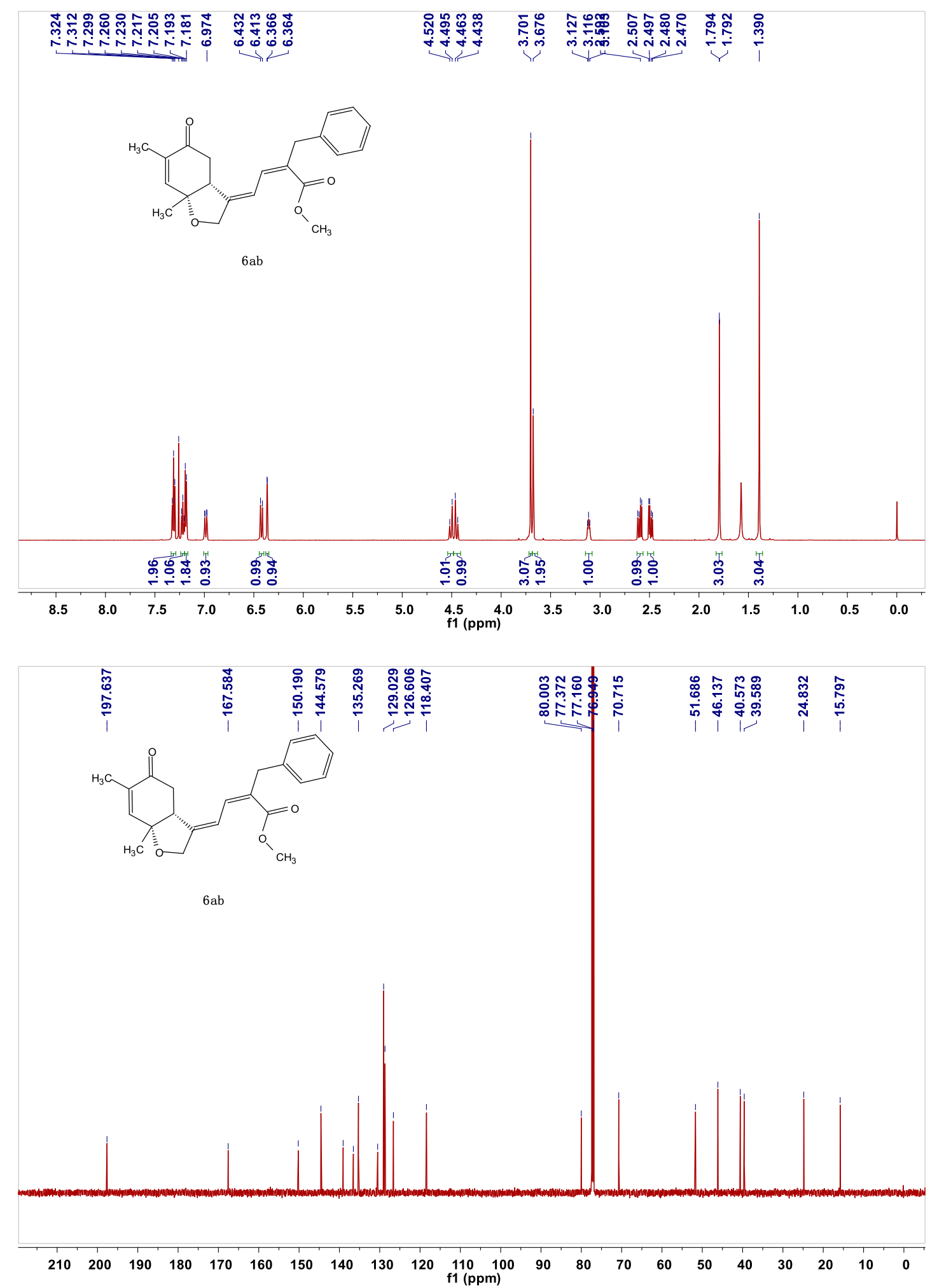

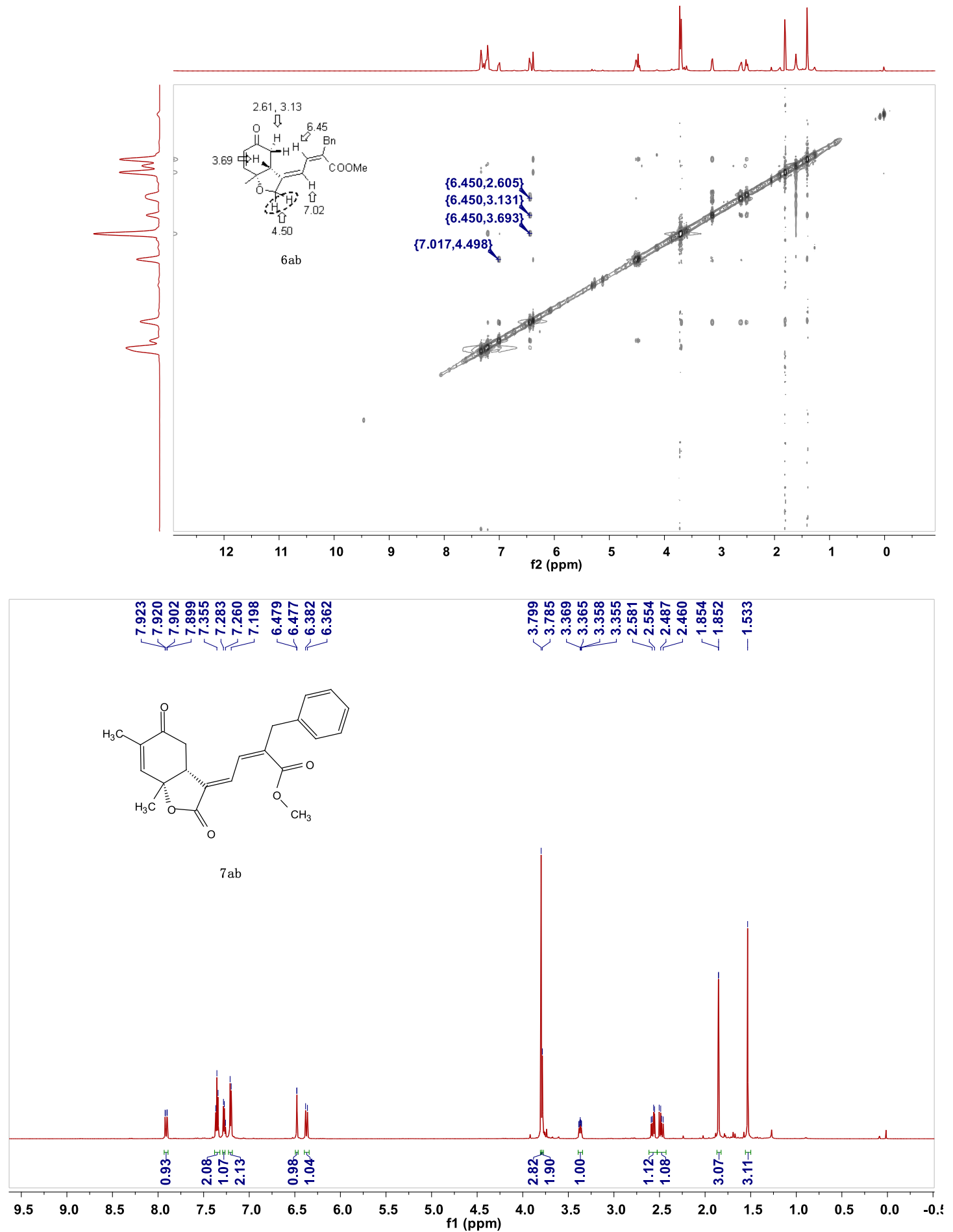


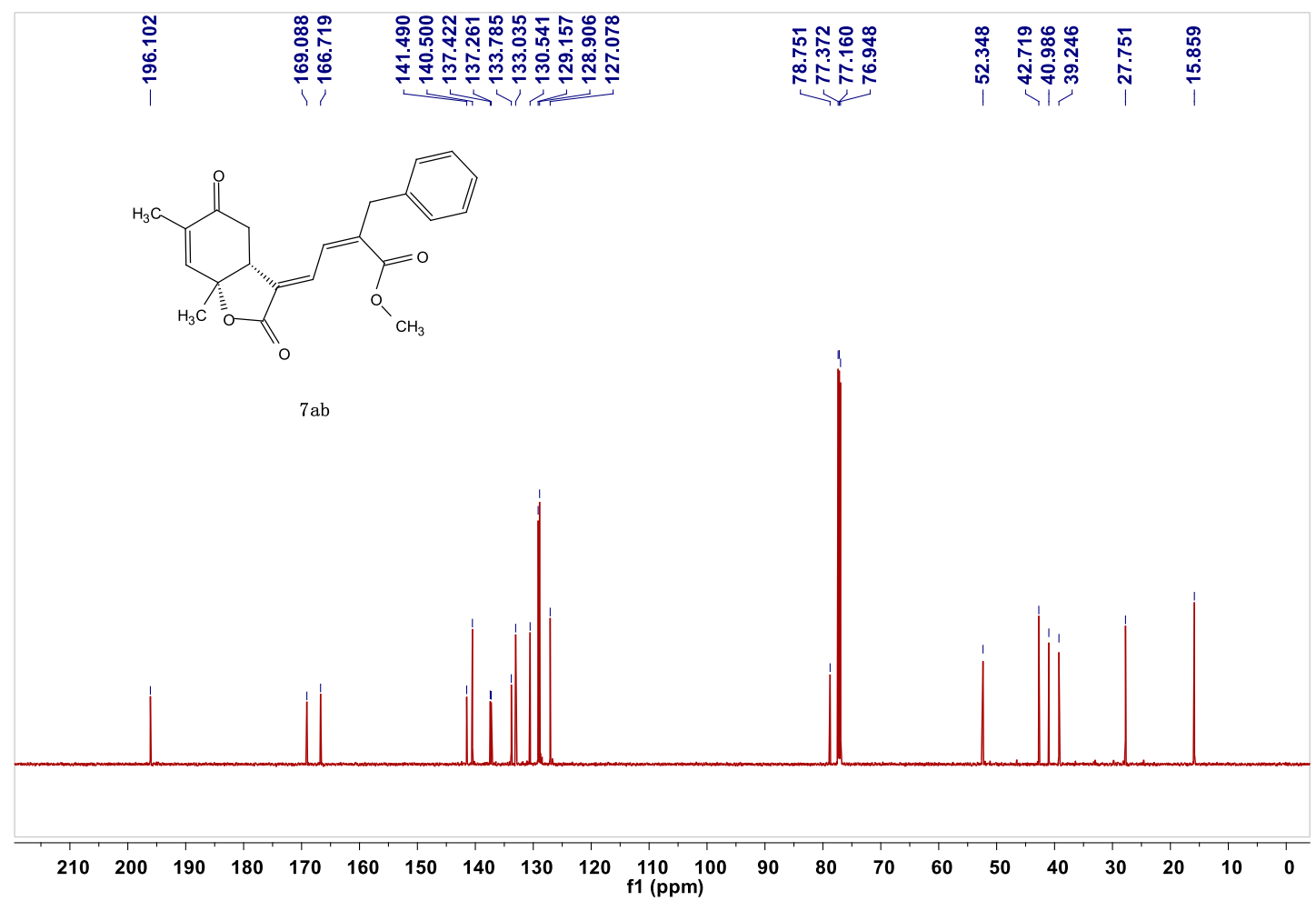




\section{References}

[1] Farley, A. J. M.; Sandford, C.; Dixon, D. J. J. Am. Chem. Soc. 2015, 137, 1599215995.

[2] Hu, X.-H.; Zhang, J.; Yang, X.-F.; Xu, Y.-H.; Loh, T.-P. J. Am. Chem. Soc. 2015, 137, 3169-3172.

[3] Fukui, Y.; Liu, P.; Liu, Q.; He, Z.-T.; Wu, N.-Y.; Tian, P.; Lin, G.-Q. J. Am. Chem. Soc. 2014, 136, 15607-15614. 